ASPECTOS GEOMETRICOS DA TEORIA DE ARNOL'D

Valdeni Soliani Fronco

Orientador: Prof. Dr. Gilberto Francisco Loibel

Dissertação apresentada ao Instituto de Ciēncias Matemáticas de São Carlos, da Universidade de São Paulo, para a obtenção do tîtulo de Mestre em Matemática. 
Aos meus pais, carla e Rafael 
AGRADECIMENTOS

Agradeço a minha esposa Carla e a meu filho Rafael, por razões ób vias.

Ao Professor Gilberto Francisco Loibel que me orientou desde o início da Graduação com muita dedicação, paciência e competência até a con clusão deste trabalho.

A Professora Izette Alves Coelho Loibel, que auxiliou na minha formação científica.

Aos Professores Carlos Biasi e Oziride Manzoli Neto pelo incen tivo e amizade.

Aos amigos do ICMSC-USP e do DME-FUEM pelo estímulo dado aos meus estudos. 
Valdeni Soliani Franco Adviser: Prof. Dr. Gilberto Francisco Loibel

In this paper we detailed some of the classification theorems from Arnold's papers, selected mainly from [1], according to the demonstration technics.

In these theorems, he classifies certain subsets of the orbits $\theta \subset \mathbb{C}^{\infty}\left(\mathbb{C}^{2}, \mathbb{C}^{1}\right)$ as well as quotients of such subsets. We also studied the topology of some of them. 
INDICE

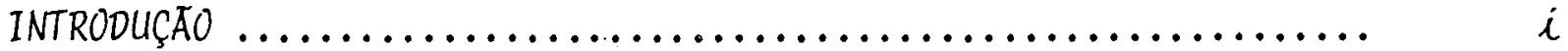

CAPITULO 0

PRELIMINARES

CAPİTULO I

ALGUMAS TECNICAS DE CLASSIFICAÇAO DE SINGULARIDADES

CAPITTULO II

A GEOMETRIA DE ALGUMAS SINGULARIDADES

BIBLIOGRAFIA 
INTRODUÇAOO

0 problema da classificação de singularidades consiste em estudar as örbitas de $C^{\infty}\left(\mathbb{C}^{m}, C^{n}\right)=\Omega_{m, n}$ sob a ação de certos grupos $(R, L, A$, etc. ver [5]). No nosso estudo olharemos para $\Omega_{2,1}$ que chamaremos simplesmente de $\Omega$, e as equivalências utilizadas serão do tipo $\mathbb{R}$.

Nos artigos de Arnol'd, principalmente em [1], encontram-se importantes contribuições para os casos $\left(\mathbb{C}^{2}, 0\right) \rightarrow(\mathbb{C}, 0)$ e $\left(\mathbb{C}^{3}, 0\right) \rightarrow(\mathbb{C}, 0)$. Em alguns dos muitos teoremas, ele classifica subconjuntos de örbitas $\theta \subset \Omega$ e em outros classifica quocientes de tais subconjuntos.

Estes estudos geralmente recaem na classificação de polinômios com caracterizações especiais (por exemplo, polinômios da forma $x^{4}+a x^{2} y^{2}+y^{4}$ ). Em muitos casos, associa a estas, classificações de objetos geométricos conhecidos.

A algumas classes desses objetos geométricos correspondem örbitas, ou seja, elementos de $\Omega$, a outros, blocos de órbitas (classes de singularidades) que são subdivididos em teoremas subsequentes.

0 estudo de uma topologia do conjunto $\Omega$ que exprimisse a "proximi dade" das órbitas é excessivamente complexo, mas certos subconjuntos do tipo $\Theta$ ou quocientes destes podem ser identificados com espaços topológicos rela tivamente simples e em certos casos estabelecido um relacionamento entre alguns desses quocientes.

No Capítulo II, estudaremos algumas situações deste tipo, relaciona dos com alguns dos teoremas de Arnol'd. Em particular, estudamos alguns casos em que refinamos os blocos dados por Arnol'd em örbitas ( $\$ 3$, Cap. II).

No Capítulo 0, relacionamos os conceitos, lemas e teoremas da teoria de Arnol'd, que serão utilizados no desenvolvimento do trabalho. 


$$
i i
$$

No Capitulo I, selecionamos doze entre os cento e cinco teoremas de Arnol'd, que aparecem em [1], de acordo com as diferentes técnicas de demons trações. Ainda neste capitulo demonstramos dois teoremas sobre funções quaseho mogêneas que além de constituirem uma técnica geral que utiliza raízes de uma álgebra de lie quase homogênea para a classificação de singularidades quasehomo gêneas, nos auxiliam na associação destas classificações com objetos geométricos conhecidos, como aparece em [1], página 48.

Pretendemos num estudo futuro, englobar os resultados aqui obtidos num es tudo mais amplo de $\Omega$. 


\section{CAPITULO 0}

\section{PRELIMINARES}

Neste Capítulo os termos que aparecem sublinhados são os conceitos que estaremos definindo.

Um ponto crítico de uma função diferenciävel é um ponto para o qual a derivada da função ē nula. Um ponto crítico é dito não-degenerado se a derivada segunda é uma forma quadrática não-degenerada.

Dizemos que dois germes de funçōes em:0 são equivalentes se um deles pode ser obtido do outro sob um difeomorfismo de $\mathbb{C}^{\mathrm{n}}$ que deixa 0 fixo.

A classe das funçōes cuja sērie de Taylor coincide em 0 para os termos de grà r com a série de Taylor de uma função dada é chamada o $\underline{r-j a t o}$ da função em 0 . 0 jato de uma função em 0 ë suficiente se ele determina o germe dessa função em 0 a menos de equivaléncia.

Denoțaremos por. th o ideal maximal do anel dos germes diferenciä veis no ponto $0 \in \mathbb{C}^{n}$. A notação $f \in \mathbb{H}^{r}$ denota que $f$ tem um zero de ordem $r$ em 0 .

\section{Lema 0.1:}

Seja $f$ um germe de uma função no ponto $0 \in \mathbb{C}^{\mathrm{n}}$. Fixamos um nümero natural $\mathrm{r}$ e assumimos que $\mathrm{f}$ satisfaz a seguinte condição:

$\left(1_{r}\right) \forall \alpha \in K^{r+1}, h_{i} \in \mathbb{K}^{2}$ tal que $\alpha=\sum_{i=1}^{n} \frac{\partial f}{\partial x_{i}} h_{i} \bmod \mathbb{H}^{r+2}$

então, qualquer função $\mathrm{f}^{\prime}$ para a qual $\mathrm{f}^{\prime}=\mathrm{f} \bmod \mathrm{H}^{\mathrm{r+1}}$ tambëm satisfaz $\left(1_{\mathbf{r}}\right)$.

\section{Lema 0.2:}

Suponhamos que o $r$-jato da função f em 0 satisfaz $\left(1_{r}\right)$, então o r-jato da função $f$ em 0 é suficiente. 
As demonstrações dos Lemas 0.1 e 0.2 estão em [3].

Consideremos o espaço aritmético $\mathbb{C}^{\mathrm{n}}$ com coordenadas $x_{1}, \ldots, x_{n}$ fixa das. Uma função $f:\left(\mathbb{C}^{n}, 0\right) \rightarrow(\mathbb{C}, 0)$ é dita ser quasehomogênea de grau d com peso $\alpha=\left(\alpha_{1}, \alpha_{2}, \ldots, \alpha_{n}\right)$ se:

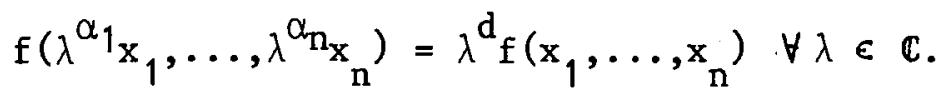

Seja f um germe de uma aplicação diferenciävel complexa, com ponto crítico isolado $0 \in \mathbb{C}^{\mathrm{n}}$. A multiplicidade $\mu$ de 0 pode ser definida como - número de pontos criticos não-degenerados no qual 0 se divide sob pequenos deslocamentos. Ela também pode ser definida como a dimensão do anel local:

$$
\mathbb{Q}_{\mathrm{f}}=\frac{\mathbb{C}\left[\left[\mathrm{x}_{1}, \ldots, \mathrm{x}_{2}\right]\right]}{\left(\partial \mathrm{f}_{1}, \ldots, \partial \mathrm{f}_{\mathrm{n}}\right)}
$$

Investigando pontos críticos de multiplicidade finita, uma função pode ser representada por jatos suficientes ou um polinômio.

Assim, a questão da classificação de singularidades isoladas se re duz a problemas algébricos concernentes à ação de grupos de lie de dimensão finita.

Uma função quasehomogēnea $f$ è dita ser não-degenerada se 0 é um ponto crítico isolado, isto é, se a multiplicidade $\mu$ do ponto crítico 0 é fi nita.

Dizemos que um monómio $x^{k}=x_{1}^{k_{1}} \ldots x_{n}^{k}$ tem grau d se $(\alpha, k)=\alpha_{1} k_{1}+$ $+\ldots+\alpha_{n} k_{n}=d$. Um polinōmio(série de potēncias, germes, funçōes) tem fill$\underline{\text { tração }}$ d se todos os seus monômios são de grau maior ou igual a d.

Os polinómios (série, germes) com fistração d forma um espaço 1 i near $E_{d}$; se $d<d^{\prime}$ então $E_{d}, \subseteq E_{d}$; a filtração de uma produto é a soma da

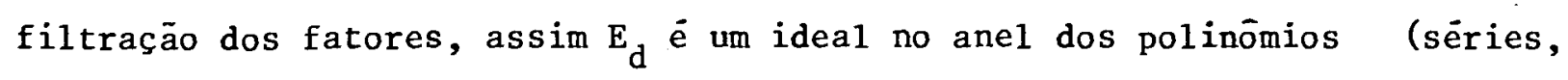
germes). Denotamos este anel por $A$, chamamos o ane 1 quociente $A / E_{d} \circ$ anel 
de d-jatos e seus elementos de d-jatos.

Um polinp̄mio (série de potēncias, germes) è dito ser semiquaseho mogēneo de grau $\underline{\text { d }}$ com peso $\underline{\alpha}$ se ele é da forma $f=f_{0}+f^{\prime}$, onde $f_{0}$ é um po linōmio (série, germe) quasehomogêneo não-degenerado de grau d e peso $\alpha$ e $\mathfrak{f}^{\prime}$ è um polinōmio (série, germe): de filtração estritamente maior que d.

Em geral, consideraremos funções quasehomogêneas de grau 1, com ex poente racional $0<\alpha_{i} \leqq 1 / 2$. Tais funções são automaticamente polinomiais. Chamamos o hiperplano $\Gamma=\left\{k: \alpha_{1} k_{1}+\ldots+\alpha_{n} k_{n}=1\right\}$ de diagonal. A diagona1 corta os eixos coordenados num segmento de comprimento $a_{i}=1 / \alpha_{i}$.

Podemos dizer então que uma função semiquasehomogênea é obtida de uma função quasehomogēnea adicionando-se monōmios cujos expontes localizam -se sobre ou acima da diagonal.

\section{Teorema 0.1:}

A multiplicidade do ponto crítico 0 de uma função semiquasehomogēnea $f$ é igual a da sua parte quasehomogênea, isto é, $\mu(f)=\mu\left(f_{0}\right)$.

A demonstração se encontra no $\$ 3$ de [2].

Por abuso de linguagem, chamamos um conjunto de $\mu$ polinômios uma base do ane 1 local de $f$ se ele torna-se uma base para $\mathbb{Q}_{f}$ sobre $\mathbb{C}$ após fatoração pelo ideal.

\section{Teorema 0.2:}

Se o sistema de monómios $e_{1}, \ldots, e_{\mu}$ é uma base do anel local da parte quasehomogênea $f_{o}$ de uma função semiquasehomogênea $f$, então este mesmo sistema de monómios é uma base do anel local de $\mathrm{E}$.

\section{Teorema 0.3:}

0 nümero de monōmios da base do anel local de uma função quasehomo gênea ou semiquasehomogēnea $f$ com grau d independe da escolha da base no a- 
nel local.

\section{Teorema 0.4:}

Uma base monomial do anel local de uma função semiquasehomogênea $f$ de peso $\left(\alpha_{1}, \alpha_{2}, \ldots, \alpha_{n}\right)$ e grau 1 , tem exatemente um gerador de grau $d_{\max }=$ $=\sum_{s=1}^{n}\left(1-2 \alpha_{s}\right)$. Todo monōmio de grau maior localiza-se no ideal $\left(\partial f_{1}, \ldots, \partial f_{n}\right)$.

As demonstrações dos Teoremas $0.2,0.3$ e 0.4 estão nos $\$ 3$ e $\$ 4$ de [2].

Consideremos o anel local das funçōes quasihomogéneas ou semiquase homogēneas $f$ de grau d. Fixamos um sistema de monōmios formando uma base para este anel.

Um monómio é dito ser superior (ou inferior, ou diagonal) se ele tem grau maior que $d$ (ou menor que $d$, ou igual a d) para um dado expoente quasehomogêneo.

Seja $e_{1}, \ldots, e_{s}$ o sistema de todos os monomios superiores da base, numa base fixa do anel local da função $f_{0}$.

\section{Teorema 0.5:}

Toda função semiquasehomogēnea com parte quasehomogènea $f_{0}$ è equivalente a uma função da forma $f_{o}+\sum c_{k} e_{k}$, onde $c_{k}$ são constantes.

Este Teorema é fundamental em värias demonstracões dos Teoremas de classificação de singularidades que faremos no Capítulo I, sua demonstração se encontra no $\$ 7$ em [2].

o grupo dos germes (ou jatos) de difeomorfismos de $\mathrm{C}^{\mathrm{n}}$ que deixa o ponto 0 fixo, atua no espaço dos germes (ou jatos) de funções em 0 . Uma classe de singularidades é um subconjunto do espaço de germes (ou jatos) de funçōes que é invariante sob esta ação. Órbitas são exemplos de classe de singularidades. Dois germes (ou jatos) são ditos equivalentes se eles per macem na mesma órbita (estas equivaléncias são as chamadas do tipo $\mathbb{R}$ ). 
Para definir Forma Normal consideremos o espaço dos polinömios $M=\mathbb{C}\left[x_{1}, \ldots, x_{n}\right]$ como um subconjundo do espaço de germes de funções $f\left(x_{1}, \ldots, x_{n}\right)$ em 0 .

Uma Forma Normal para uma classe $K$ de funções é dada pela aplicação $\phi: B \rightarrow M$ de um espaço linear de dimensão finita de parāmetros $B$ no espaço dos polinômios, para o qual as três condiçoes abaixo se verificam:

(1) $\phi(B)$ intercepta toda örbita de K;

(2) a imagem inversa em $B$ de cada órbita é finita;

(3) a imagem inversa de todo complemento para $k$,está contida em alguma hiper superficie própria em B.

Uma Forma Normal é dita polinomial (respectivamente afim) se a aplicação $\varnothing$ é polinomial (respectivamente linear e não homogênea). Uma Forma Normal afim é chamada simples se $\phi$ tem a forma:

$\phi\left(b_{1}, \ldots b_{r}\right)=\psi_{0}+b_{1} x_{1}^{m_{1}}+\ldots+b_{r} x_{r}^{m_{r}}$, onde $\psi_{0}$ é um polinōmio fixado, os $b_{i}$ são números e os $\mathrm{x}^{\mathrm{m}^{\mathrm{i}}}$ são monômios.

Dois germes $\mathrm{f}:\left(\mathbb{C}^{\mathrm{n}}, 0\right) \rightarrow(\mathbb{C}, 0)$ e $g:\left(\left(\mathbb{C}^{\mathrm{m}}, 0\right) \rightarrow(\mathbb{C}, 0)\right.$ são ditos serem estavelmente equivalentes se eles tornam-se equivalentes apōs adiçāo de formas quadráticas não-degeneradas (por exemplo: $f(x)=x^{3}$ é estavelmente equivalente a $\left.g(x, y)=x^{3}+y^{2}\right)$. Se duas funções com o mesmo nümero de variáveis são estavelmente equivalentes, então elas são equivalentes.

o corank do germe de uma função em um ponto critico è a dimensão do espaço nulo de sua derivada segunda. Toda funçao de corank $\mathrm{r}$ è estavelmen te equivaelnte a uma função de $r$ variäveis (Lema de Morse generalizado). Na classificação a menos de equivaléncia estāvel geralmente tomaremos formas nor mais com o nümero de variāveis igual ao corank.

0 grupo de difeomorfismos quasehomogēneos de peso $\alpha$ è o grupo de germes de difeomorfismos de $\mathbb{C}^{\mathfrak{n}}$ em 0 levando qualquer função quasehomogénea 
de peso $\alpha$ e grau d em uma função de mesmo grau. A àlgebra de Lie deste grupo é chamado a ālgebra quasehomogènea e será denotada por $\pi(\alpha)$.

0 espaço dos expoentes de funções de $x_{1}, \ldots x_{n}$ è o espaço aritmético $\mathbb{C}^{n}$ cujos pontos $m$ com coordenadas inteiras não negativas são os expoentes dos monómios $\mathrm{x}^{\mathrm{m}}$.

0 suporte de funções quasehomogêneas de grau d e peso $\alpha$ é o con junto de todos os pontos inteiros não-negativos m no plano $(m, \alpha)=d$. 0 su porte e dito ser completo se ele não estä contido em um subespaço afim de $\mathbb{C}^{\mathbf{n}}$ de dimensão menor que $\mathrm{n}-1$.

As raizes da algebra !quasehomogênea $\alpha(\alpha)$ são todos os vetores não nulos $m$ do espaço de expoentes que localizam-se no plano $(m, \alpha)=0$ e tem a

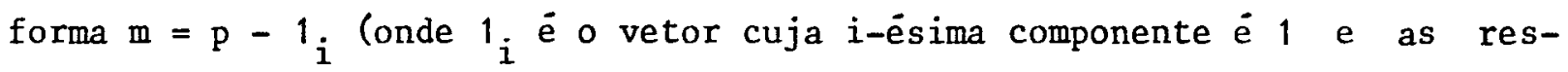
tantes iguais a 0 e o vetor $p$ tem componentes interras não negativas)

Em outras palavras, m é uma raiz se $x^{\mathrm{p}_{\partial}}{ }_{i}$ é um campo vetorial monomial de $x(\alpha)$ distinto de $x_{i}{ }^{\partial}$. Observemos que $i$ pode ser recuperado de uma raiz $m$, desde que $m$ tem precisamente uma coordenada negativa $m_{1}=-1$ (nem to das componetes de $m$ pode ser não negativas, pois $(m, \alpha)=0)$.

Queremos agora para finalizar, fixar algumas notações que aparecerão nos teoremas de classificação.

Por $j_{k} f$ entende-se o $k-j a t o$ de $f$ em 0 (ou o polınómio de Taylor de ordem $k$ em 0 ).

$j_{\left\{x^{\prime}\right\}} f$ e o quase $j$ ato de $f$ em 0 , definido pelo monómio $x^{m_{i}}$ (ou o correspondente polınōmio de Taylor).

$j_{\{x} m_{1\}} f \approx g$ denota equivalência quasehomogênea de jatos (ou polinō mios de Taylor). 


\section{* Observações:}

1. Um sistema de $n$ monômios $\left\{x^{m^{i}}\right.$ em $x_{1}, \ldots, x_{n}$ com expoentes independentes $\mathrm{m}_{\mathrm{i}} \in \mathbb{2}^{\mathrm{n}} \subset \mathbb{R}^{\mathrm{n}}$ define um hiperplano $\Gamma=\{\mathrm{m}:(\alpha, \mathrm{m})=1\}$. Se todas as com ponestes $\alpha_{i}$ do vetor $\alpha$ são positıvas, então $\alpha$ é chamado o tipo de quasehomogeneidade e o número $(\alpha, m)$ é a ordem do monômio $x^{m} \cdot 0$ polinômios $f=\sum_{\mathrm{f}} x^{m} \dot{e}$ quasehomogēneo de grau d e tipo $\alpha$ se $(\alpha, m)=d \quad \forall m: f_{m} \neq 0$. 0 tipo de qua sehomogeneidade define em $\left.\mathbb{C}\left[\mathrm{x}_{1}, \ldots, \mathrm{x}_{\mathrm{n}}\right]\right]$ uma graduação e uma filtração decrescente $\xi_{0} \supset \xi_{1} \supset \ldots$, onde:

$$
\varepsilon_{\mathrm{d}}=\left\{\mathrm{f}:(\alpha, \mathrm{m}) \geqq \mathrm{d}, \forall \mathrm{m} \operatorname{com} \mathrm{f}_{\mathrm{m}} \neq 0\right\}
$$

2. O espaço quociente $\xi_{\mathrm{o}} / \varepsilon_{\mathrm{d}}, \mathrm{d}>1$, e- chamado um espaço de quasejatos definido pelos monómios $\left\{\mathrm{x}^{\mathrm{m}} \mathrm{\}}\right.$ (ou definido pelo tipo de quasehomogeneidade $\alpha$ ). Para um sistema de coordenadas fixado, podem ser identificados çom polinômios cujos monōmios são todos de ordem maior que 1 (um). 


\section{ALGUMAS TECNICAS DE CLASSIFICAÇAOO DE SINGULARIDADES}

Neste capitulo demonstraremos alguns teoremas de classificação de singularidades utilizando diversas tëcnicas que aparecem basicamente em dois artigos de V.I.Arnol'd: "Critical points of smooth functions and their normal forms" e "Normal forms of functions in neighbourhoods of degenerate critical points" citados em nossa bibliografia. Os prê-requisitos ne cessärios ou foram dados no Capítulo 0 ou serão citados antes das demonstra ções de cada teorema.

Os teoremas numerados de 1.1 a 1.12 foram retirados de uma sêrie de 105 teoremas que aparecem no artigo [1], e antes de enunciarmos cada um desses teoremas colocaremos entre parenteses o número do teorema no referido artigo. Eles foram selecionados devido as diferentes técnicas de demonstração. Algumas dessas demonstrações não existem no artigo, outras fizemos com mais detalhes do que as feitas no artigo.

\section{Teomema $1.1(2):$}

Se corank $f \leqq 1 \Rightarrow f \in A_{k}(k \geqq 1)$, onde $A_{k}: x^{k+1}(k \geqq 1)$

Demonstração:

Em [3], Lema 4.1 è demonstrado que numa vizinhança de 0 , existe um sistema de coordenadas $x, y$ no qual $\underline{f}$ pode ser escrito da forma:

$f=\Phi(x)+Q(y)$ onde $Q$ é uma forma quadrätica não degenerada e $\operatorname{dim}\{x\}=\operatorname{corank} f$.

No nosso caso corank $f \leqq 1$. Em virtude do Lema 0.2 o $(k+1)$-jato: (*) $c x^{k+1}+y_{1}{ }^{2}+\ldots+y_{n-1}{ }^{2} \quad(c \neq 0)$ é suficiente, isto seque da fatora ção de todos os monômios em $x, y$ de grau $k+2$. 
$x^{k+2}=(k+1) c x^{k} \cdot\left(\frac{x^{2}}{c(k+1)}\right), \operatorname{com} \frac{x^{2}}{c(k+1)} \epsilon+h^{2}$

Substituindo $x$ por $x^{\prime}=|c|^{1 /(k+1)} x$ em $(*)$, obtemos $f \in A_{k}$.

Teorema $1.2(3):$

Se $j_{2} f=0 \Rightarrow$ um dos quatro casos ocorrem:

$j_{3} f \approx x^{2} y+y^{3} ; j_{3} f \approx x^{2} y ; j_{3} f \approx x^{3}$ ou $j_{3} f=0$

\section{Demonstração:}

Como o 2-jato de $\underline{\mathrm{f}}$ è nulo, temos que o 3-jato determina uma for ma cúbica no plano tangente. Uma forma cúbica em $\mathbb{C}^{2}$ è reduzida por uma mu dança de variāveis em um dos seguintes tipos: $x^{2} y+y^{3} ; x^{2} y ; x^{3}$ ou 0 . De fa to:

$$
\begin{aligned}
& j_{3} f=a_{0} x^{3}+a_{1} x^{2} y+a_{2} x y^{2}+a_{3} y^{3} \text { ou ainda } \\
& j_{3} f=\left(\alpha_{1} x-\beta_{1} y\right)\left(\alpha_{2} x-\beta_{2} y\right)\left(\alpha_{3} x-\beta_{3} y\right)
\end{aligned}
$$

10 Caso: $a_{0}=a_{1}=a_{2}=a_{3}=0$, temos $j_{3} f=0$

20 Caso: $\alpha_{1}=\alpha_{2}=\alpha_{3}$ e $\beta_{1}=\beta_{2}=\beta_{3}$, fazemos $n=\alpha_{1} x-\beta_{1} y$ e assim $\mathrm{j}_{3} \mathrm{f}=\mathrm{n}^{3}$ ou $\mathrm{j}_{3} \mathrm{f} \approx \mathrm{x}^{3}$

30 Caso: Se $\alpha_{1}=\alpha_{2}$ e $\beta_{1}=\beta_{2}$, fazemos $\eta=\alpha_{1} \mathrm{x}-\beta_{1} \mathrm{y}$ e $\xi=\alpha_{3} \mathrm{x}-\beta_{3} \mathrm{y}$

$$
\text { Assim } j_{3} f=n^{2} \xi \text { ou } j_{3} f \approx x^{2} y
$$

49 Caso: Todas as raízes são distintas

$$
\text { Podemos escrever } j_{3} f=x y(\alpha x-\beta y)=\alpha x^{2} y-\beta x y^{2} \text { e por dilata - }
$$

ção ou contração de coordenadas obtemos $x^{2} y-x y^{2}=x y(x-y)$. Consideremos um novo sistema $\xi=x-y$ e $n=x+y \Rightarrow x=\frac{\eta+\xi}{2}$ e $y=\frac{n-\xi}{2} \Rightarrow j_{3} f=$ $=\frac{n^{2}-\xi^{2}}{4}(\xi)=\frac{n^{2} \xi}{4}-\frac{\xi^{3}}{4}$ fazendo uma dilatação $j_{3} f \approx x^{3}+x^{2} y$ 
Teorema $1.3(5)$ :

Se $j_{3} f=x^{2} y \Rightarrow f \in D_{k} \quad(k>4)$, onde $D_{k}: x^{2} y+y^{k-1}(k \geqq 4)$

Demonstração:

Se $j_{3} f=x^{2} y$ então o s-jato de $\underline{f}$ tem a forma $x^{2} y+\phi_{s}(x, y), \phi_{s} \dot{e} u m$ polinōmio homogêneo de grau $s$. Representamos $\phi_{S}$ na forma:

$\phi_{s}=a y^{s}+2 b x y^{s-1}+x^{2} \psi(x, y)$, onde $\psi \epsilon t^{s-2}$

Substituindo $\mathrm{x}-\mathrm{by}^{\mathrm{s}-2}=\mathrm{x}_{1}$ e $\mathrm{y}-\psi(\mathrm{x}, \mathrm{y})=\mathrm{y}_{1}$ reduzimos $\underline{\mathrm{f}} \dot{\mathrm{a}}$ forma $\mathrm{f}=\mathrm{x}_{1}{ }^{2} \mathrm{y}_{1}+\mathrm{ay}_{1} \mathrm{~s} \bmod \mathrm{k}^{\mathrm{s}+1}$ (pois $\mathrm{k}^{2 \mathrm{~s}-3} \subset \mathrm{H}^{\mathrm{s}-1}$ para $\mathrm{s}>3$ ). Segue que dois casos são possiveis: $a=0$ ou $a \neq 0$. Se $a=0$, então o $s-j a t o$ da função é reduzí do à forma $x_{1}{ }^{2} y_{1}+\phi_{S+1}\left(x_{1}, y_{1}\right)$ e podemos repetir o argumento anterior, continuando até chegarmos a $a \neq 0$. Se $a \neq 0$ nunca ocorre então a singularidade tem codimensão infinita.

Consideremos o $\underline{s}$ tal que $a \neq 0$, então o germe da função $\underline{\mathbf{f}}$ em zero será equivalente ao germe de $x^{2} y+a y^{s}$. De fato: basta verificar que o $s-j \underline{a}$ to $x^{2} y+\frac{a y}{s}(s>3, a \neq 0)$ é suficiente.

$$
\begin{aligned}
& x^{\alpha} y^{\beta}=(2 x y)(h(x, y)), h \in \pi^{2} \text { para } \alpha+\beta=s+1>3(\alpha>0, \beta>0) \\
& x^{s+1}=\left(x^{2}+a y^{s-1}\right)\left(x^{s-1}\right)+2 x y\left(\frac{-x^{s-2} y^{s-2}}{2 a^{-1}}\right)\left(x^{s-1} \epsilon t^{2},(x y)^{s-2} \in \pi^{2}\right) \\
& y^{s+1}=\left(x^{2}+a y^{s-1}\right)\left(\frac{y^{2}}{a}\right)^{+2 x y}\left(\frac{-x y}{2}\right)\left(y^{2} \in \pi^{2},\left(\frac{-x y}{2}\right) \in \nVdash^{2}\right)
\end{aligned}
$$

Para converter $x^{2} y+\frac{a y^{s}}{s}$ em $x^{2} y+y^{s}$ basta substituir $x_{1}=p x e$ $\mathrm{y}_{1}=\mathrm{qy}$.

\section{Teorema $1.4\left(6_{k}\right)$ :}

Se $j_{x}{ }^{3}, y^{3 k} f(x, y)=x^{3} \Rightarrow$ ocorre um dos quatro casos:

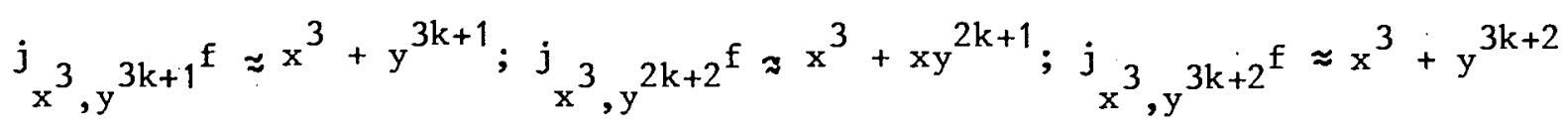
ou $j_{x^{3}, y} 3 k+2 f \approx x^{3}$. 


\section{Demonstração:}

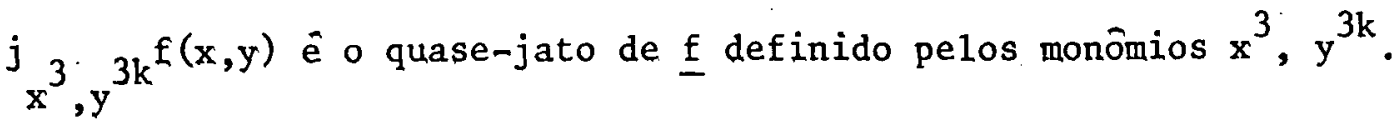
Assim o suporte da função, localiza-se estritamente acima da 1inha que liga os pontos $(3,0)$ e $(0,3 k)$

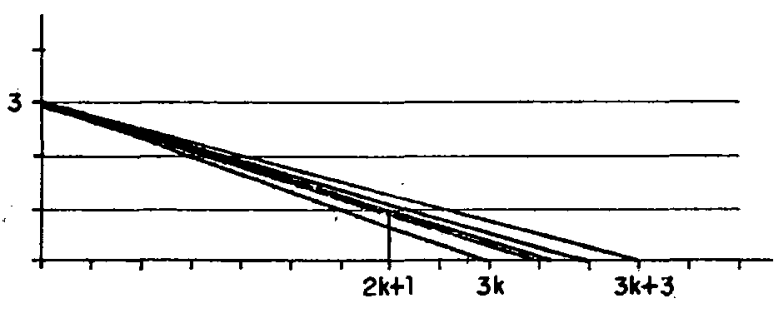

Colocamos uma régua sobre esta linha, fixamos $(3,6)$ e movemos a outra extremidade para cima, encontrando expoentes de monômios não nulos.

Como podemos ver pela figura, dentro do triângulo entre as retas ligando $(3,0)$ a $(0,3 k)$ e a $(0,3(k+1))$ existem ao todo três pontos reticula dos (no quadrante positivo) a saber, $(0,3 k+1) ;(1,2 k+1)$ e $(0,3 k+2)$.

Se o coeficiente de $\mathrm{y}^{3 k+1}$ è diferente de 0 , então temos o primeiro caso do teorema. Se o coeficiente de $y^{3 k+1}$ e 0 , mas o de $x y^{2 k+1}$ é diferente

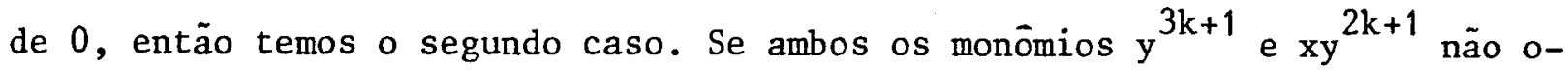
corre em $\underline{f}$, mas $y^{3 k+2}$ sim, então temos o terceiro caso. Se os coeficientes de todos os trés monómios $y^{3 k+1}, x^{2 k+1}$ e $y^{3 k+2}$ são nulos, então temos o outro caso.

Teorema $1.5\left(7_{k}\right)$ :

Se $j_{x^{3}, y} 3 k+1=x^{3}+y^{3 k+1} \Rightarrow f \in E_{6 k}$, onde $E_{6 k}: x^{3}+y^{3 k+1}+a x y^{2 k+1}$
$\operatorname{com} a=a_{0}+\ldots+a_{k-2} y^{k-2}$

Demonstração:

Pelo Lema 0.2 , a função quasehomogênea $f_{0}=x^{3}+y^{3 k+1}$ tem $03 k+1-$ -jato suficiente, trivialmente.

$E_{6 k}: x^{3}+y^{3 k+1}+a x y^{2 k+1}$, onde $a=a_{0}+a_{1} y+\ldots+a_{k-2} y^{k-2}$ 
( e $a=0$, para $k=1$ )

0 Teorema 0.5 garante que toda função semiquasehomogênea $f$ é equivalente a uma função da forma $f_{0}+\sum c_{k} e_{k}$, onde $c_{k}$ são constantes $e e_{1}, e_{2}$, ...,e és o sistema de monómios superiores da base, numa base monomial fixa do anel local da função $f$.

Monōmios superiores devem ter grau superior a $9 k+3$ (grau de $f$ ). Então o menor grau será $9 k+4$. Monômios contendo só $x$ para ter grau superior a $9 k+3$ deve ter expoente superior a 3 que está assim contido no ideal. Monômios contendo y puro, para ter grau superior a $9 k+3$ deve ter expoente acima de $3 k+1$ e assim estará no ideal. Não se deve ter monômios que contenham $x^{2}$, pois estariam claramente no ideal, restam então monōmios que contém $\mathrm{x}$ e $\mathrm{y}^{\mathrm{p}}$, além disso $(3 k+1)+3 p \geqq 9 k+4 \Rightarrow 3 p \geqq 6 k+3 \Rightarrow p \geqq 2 k+1$.

Para calcularmos o limite de $\mathrm{p}$ devemos calcular $\mathrm{d}_{\max }$ (ver Teorema $0.4)$.

$$
\begin{aligned}
\mathrm{d}_{\max } & =\mathrm{n}-2 \sum \alpha_{i}=2-2((1 / 3)+(1 /(3 \mathrm{k}+1)))=2-(6 \mathrm{k}+8) /(9 \mathrm{k}+3)= \\
& =(18 \mathrm{k}+6-6 \mathrm{k}-3) /(9 \mathrm{k}+3)=(12 \mathrm{k}-2) /(9 \mathrm{k}+3)
\end{aligned}
$$

Queremos então que $9 k+4+3 n=12 k-2 \Rightarrow_{n}=k-2$, onde $n$ é o maior expoente de y que podemos multiplicar $x^{2 k-1}$.

$$
\text { Portanto } \mathrm{f}=\mathrm{x}^{3}+\mathrm{y}^{3 \mathrm{k}+1}+a x \mathrm{y}^{2 \mathrm{k}+1} \text {, onde } a=a_{0}+\mathrm{a}_{1} \mathrm{y}+\ldots+\mathrm{a}_{\mathrm{k}-2} \mathrm{y}^{\mathrm{k}-2} \text {. }
$$

No artigo [1], Arnol'd formula teoremas gerais sobre funçōes qua sehomogêneas (Teoremas $A$ e $B$ ) que além de nos auxiliar na classificação das singularidades nos fornece uma maneira de identificarmos problemas de clas sificação com problemas geométricos.

$\mathrm{Na}$ sequência enunciaremos esses teoremas, fazendo suas demonstra cões com mais detalhes que no artigo. Citaremos em seguida alguns corolärios importantes.

Seja $M \subset \mathbb{C}^{\mathrm{r}} \subset \mathbb{C}^{\mathrm{n}}$ um sistema de raizes de $\alpha(\alpha)$ gerando o plano $\mathbb{C}^{\mathrm{r}}$ 
em $\mathbb{C}^{\mathrm{n}}(0 \leqq \mathrm{r} \leqq \mathrm{n}-1)$. A cada $\mathrm{m} \in \mathrm{M}$ associamos um vetor $e_{m}$ de uma base veto rial em $\mathbb{C}^{\nu}(\nu$ é o nümero de raízes). Consideremos o espaço vetorial r-di mensional $\mathrm{H}=\operatorname{Hom}\left(\mathbb{C}^{\mathrm{r}}, \mathbb{C}\right)$ e a soma $\operatorname{direta} \mathfrak{b}=\mathrm{H} \oplus \mathbb{C}^{\nu}$.

\section{Lema 1.1:}

Ao espaço $\zeta=H \oplus C^{\nu}$ pode ser dado a seguinte estrutura de álgebra de Lie:

(1) $\left[h^{\prime}, h^{\prime \prime}\right]=0 \quad \forall h^{\prime}, h^{\prime \prime} \in H$

(2) $\left[\mathrm{h}, \mathrm{e}_{\mathrm{m}}\right]=(\mathrm{h}, \mathrm{m}) \mathrm{e}_{\mathrm{m}} \forall \mathrm{h} \in \mathrm{H}, \mathrm{m} \in \mathrm{M}$

(3) $\left[e_{m_{1}}, e_{m_{2}}\right]=N_{m_{1}}, m_{2} e_{m_{1}}+m_{2}$, desde que $m_{1}+m_{2}, \neq 0$ e onde

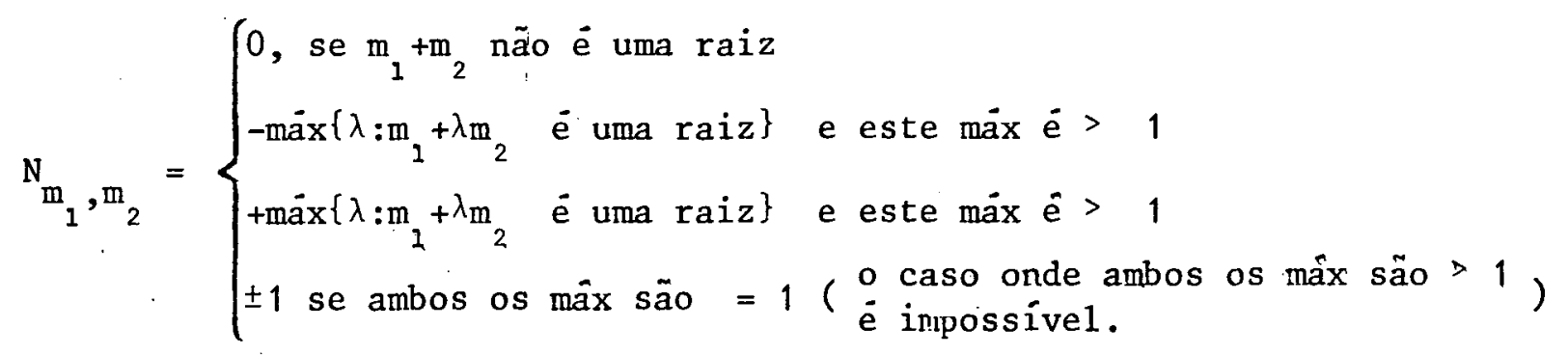

(4) $\left[e_{m}, e_{-m}\right]=h_{m}$ onde $h_{m} \in H$ muda de sinal sọb a reflexão de $C^{r}$ que pre serva $M$ e troca $m$ por -m normalizado pela condição $h_{m}(m)$

= 2 (uma tal reflexão existe e é única para qualquer par de raízes opostas).

A àlgebra de Lie quasehomogênea $x(\alpha)$ è isomörfa a soma direta da álgebra de Lie 6 (para alguma escolha de sinal \pm em (3)) e uma älgebra (comutativa) trivial $x(\alpha) \approx b \oplus C^{n-r}$.

\section{Demonstração:}

Consideremos uma base monomial para $\alpha(\alpha)$ sobre $\mathbb{C}$ e escrevemos os monômios da base como segue:

$$
h^{\prime}=x_{i}{ }^{\partial} \text { e } \quad e_{m}=x^{m} x_{i}{ }^{2}
$$

(1) Sejam h', $h^{\prime} \in H \Rightarrow h^{\prime}=x_{i}{ }^{\partial}$ e $h^{\prime \prime}=x_{j}{ }^{\partial} j$

$$
\left[h^{\prime}, h^{\prime}\right]=\left[x_{i}{ }^{2}, x_{j}{ }^{2}\right]=0 \quad \text { (trivialmente) }
$$


(2) Sejam $h \in H$ e $m \in M \Rightarrow$

$$
\begin{aligned}
& {\left[h, e_{m}\right]\left(x_{1}^{\alpha_{1}} \ldots x_{n}^{\alpha_{n}}\right)=\left[x_{i} \partial, x^{m_{x}} x_{j}{ }_{j}\right]\left(x_{1}^{\alpha_{1}} \ldots x_{n}^{\alpha_{n}}\right)=} \\
& =\left(x_{i} \partial_{i \circ} x^{m} x_{j}\right)\left(x_{1}^{\alpha_{1}} \ldots x_{n}^{\alpha_{n}}\right)-\left(x^{m} x_{j}{ }_{j} \circ x_{i}{ }^{\partial}{ }_{i}\right)\left(x_{1}^{\alpha_{1}} \ldots x_{n}^{\alpha_{n}}\right)=
\end{aligned}
$$

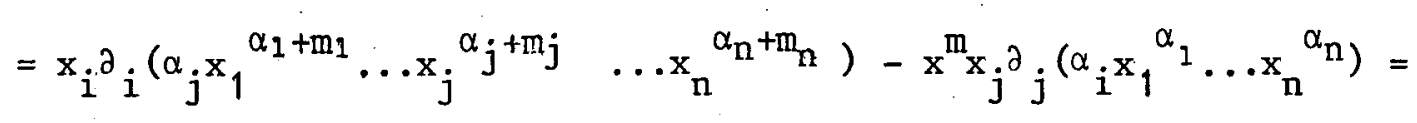

$$
\begin{aligned}
& =\alpha_{j}\left(\alpha_{i}+m_{i}\right) x_{1}{ }^{\alpha_{1}+m_{1}} \ldots x_{r}{ }^{\alpha_{r}+m_{r}}-\alpha_{i} \alpha_{j} x_{1}{ }^{\alpha_{1}+m_{1}} \ldots x_{n}^{\alpha_{n}+m_{n}}= \\
& =\alpha_{j} m_{i} x_{1}{ }^{\alpha}+m_{1} \ldots x_{n}^{\alpha_{n}+m_{n}}=m_{i} x^{m_{x} x_{j}{ }^{2}}=m_{i} e_{m}=(h, m) e_{m}
\end{aligned}
$$

(3) $\left[e_{m}, e_{m^{\prime}}\right]\left(x_{1}^{\alpha}{ }^{\alpha} \ldots x_{n}^{\alpha}\right)=\left[x^{m} x_{i}{ }^{\alpha}, x^{m^{\prime}} x_{j}{ }^{2} j\right]\left(x_{1}{ }^{\alpha}{ }_{1} \ldots x_{n}^{\alpha}{ }^{\alpha}\right)=$

$=x_{i}^{i m} x_{i}{ }_{i}\left(\alpha_{j} x_{1}{ }^{\alpha_{1}+m_{1}^{\prime}} \ldots x_{n}{ }^{\left.\alpha_{n}+m_{n}^{\prime}\right)}-x^{m^{\prime}} x_{j}{ }^{\partial}{ }_{j}\left(\alpha_{i} x_{1}{ }^{\alpha_{1}+m_{1}} \ldots x_{n}{ }^{\alpha_{n}+m_{n}}\right)=\right.$

$=\left(\alpha_{j} m_{i}^{\prime}-\alpha_{i} m_{j}\right) x_{1}{ }^{\alpha_{1}+m_{1}+m_{1}^{\prime}} \ldots x_{i}{ }^{\alpha_{i}+m_{i}+m_{1}^{\prime}} \ldots x_{j}{ }^{\alpha_{j}+m_{j}+m_{j}^{\prime} \ldots x_{n} \alpha_{n}+m_{n}+m_{n}^{\prime}}$

Como $\mathrm{m}$ e $\mathrm{m}^{\prime}$ são raízes

$\mathrm{m}=\left(\mathrm{m}_{1}, \ldots,-1, \ldots, \mathrm{m}_{j}, \ldots, \mathrm{m}_{\mathrm{n}}\right)$
$\mathrm{m}^{\prime}=\left(\mathrm{m}_{1}^{\prime}, \ldots, \mathrm{m}_{i}^{\prime}, \ldots,-1, \ldots, \mathrm{m}_{\mathrm{n}}^{\prime}\right)$

Tomemos $m+\lambda m^{\prime}=\left(m_{1}+\lambda m_{1}^{\prime}, \ldots,-1+\lambda m_{i}^{\prime}, \ldots, m_{j}+(-\lambda), \ldots, m_{n}+\lambda m_{n}^{\prime}\right)$

Para ser raiz devemos ter:

$$
\begin{aligned}
& -1+\lambda m_{i}^{\prime}=-1 \text { e } m_{j}-\lambda \geqq 0 \text { ou } \\
& -1+\lambda m_{i}^{\prime} \geqq 0 \text { e } m_{j}-\lambda=-1
\end{aligned}
$$

19 Caso: $-1+\lambda m_{i}^{\prime}=-1$ e $m_{j}-\lambda \geqq 0 \Rightarrow m_{i}^{\prime}=0$ e $m_{j} \geqq \lambda$. Assim

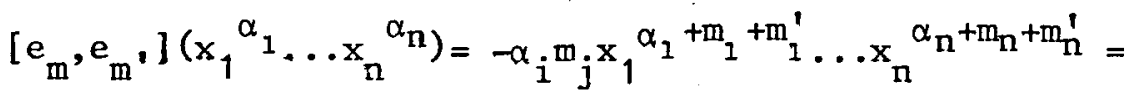

$$
\begin{aligned}
& =-m_{j} e_{m+m^{\prime}}=-m a ̄ x x\left\{\lambda: m+\lambda m^{\prime} e^{-} \text {uma raiz }\right\} e_{m+m^{\prime}}
\end{aligned}
$$

29 Caso: $-1+\lambda \mathrm{m}_{i}^{\prime} \geqq 0$ e $\mathrm{m}_{j}-\lambda=-1 \Rightarrow \lambda \mathrm{m}_{i}^{\prime} \geqq 1$ e $\lambda=\mathrm{m}_{j}+1$

Se tivermos pesos $\left(\beta_{1}, \beta_{2}, \ldots, \beta_{n}\right)$, obteremos:

a. $\beta_{1} m_{1}+\beta_{2} m_{2}+\ldots-\beta_{i}+\ldots+\beta_{j} m_{j}+\ldots+\beta_{n} m_{n}=0$ 


$$
\begin{aligned}
\Rightarrow & \beta_{i}=\beta_{1} m_{1}+\ldots+\beta_{j} m_{j}+\ldots+\beta_{n} m_{n}^{\prime} \\
& \text { b. } \beta_{1} m_{1}^{\prime}+\beta_{2} m_{2}^{\prime}+\ldots+\beta_{i} m_{i}^{\prime}+\ldots+\left(-\beta_{j}\right)+\ldots+\beta_{n} m_{n}^{\prime}=0 \Longrightarrow \\
\Rightarrow & \beta_{j}=\beta_{1} m_{1}^{\prime}+\ldots+\beta_{i} m_{i}^{\prime}+\ldots+\beta_{n} m_{n}^{\prime}
\end{aligned}
$$

Como $m_{i}^{\prime}$ são inteiros não negativos para $i \neq j$ então para que $\lambda m_{i}^{\prime} \geqq 1$, devemos ter $\lambda \geqq 1$ e $\mathrm{m}_{i}^{\prime} \geqq 1$.

(A) Caso $\lambda^{\prime}>\mathrm{m}_{\mathbf{j}}>0 \quad \begin{array}{r}\text { a. } \\ \quad \beta_{i} \geqq \beta_{j}\end{array}$

$$
\begin{aligned}
\left(A_{1}\right) B_{i}>B_{j} & \left.\Rightarrow m_{i}^{\prime}=0 \text { (absurdo pois por hipótese } m_{i}^{\prime} \geqq 1\right) \\
\left(A_{2}\right) \quad B_{i}=B_{j} \Rightarrow m & \Rightarrow=(0, \ldots, 0,-1,0, \ldots, 0,1,0, \ldots, 0) \\
m^{\prime} & =(0, \ldots, 0,1,0, \ldots, 0,-1,0, \ldots, 0)
\end{aligned}
$$

Assim: $\left[e_{m}, e_{m^{\prime}}\right]=\left(\alpha_{j}-\alpha_{i}\right) x_{1} \alpha_{1}+m_{1}+m_{1}^{\prime} \ldots x_{n} \alpha_{n}+m_{n}+m_{n}^{\prime}=h_{j}-h_{i}=h_{m}$.

(B) Caso $\lambda=1 \underset{\text { hip. }}{\Rightarrow} m_{j}=0$ e $\left[e_{m}, e_{m^{\prime}}\right]=\alpha_{j} m_{i}^{\prime} x_{1}{ }^{\alpha}+m_{1}+m_{1}^{\prime} \ldots x_{n}{ }_{n}+m_{n}+m_{n}^{\prime}=$ $=\max \left\{\lambda: m^{\prime}+\lambda m\right.$ é uma rai $\left.z\right\} e_{m+m^{\prime}}$.

Assim h e e ${ }_{m}$ satisfazem (1)-(4)

Sabemos pela construção de (4) que $h_{m}(-m)=-h_{m}(m)$. Logo

$h_{m}(M)=2 e h_{m}(v)=0 \forall v \in M^{1}$

$\left[e_{m}, e_{-m}\right]=-\left[e_{-m}, e_{m}\right]=h_{m} \in H^{r}=\prod_{i=1}^{r}\left[x_{i} \partial\right]=\operatorname{Hom}\left(\mathbb{C}^{r}, \mathfrak{C}\right)$

o espaço $\mathbb{C}^{\mathfrak{r}}$ e a métrica são invariantes relatios a todas as permu tações de coordenadas com pesos iguais $\left(\alpha_{i}=\alpha_{j}\right)$. Portanto o complemento ortogonal $\mathbf{c}^{\mathrm{n}-\mathrm{r}}$ para $\mathbb{C}^{\mathrm{r}}$ em $\mathbb{C}^{\mathrm{n}}$ é também invariante. Representamos o espaço linear $x(\alpha)$ como $x(\alpha)=H^{r} \oplus K^{\nu} \oplus H^{n-r}$, onde $H^{r}$ consiste daquelas funçöes lineares $h$ em $\mathbb{C}^{n}$ que se anulam em $\mathbb{C}^{n-r} ; H^{n-r}$ é constituido por funções $h$ em $\mathbb{C}^{n}$ que se anulam em $\mathbb{C}^{r} ; K^{\nu}$ as combinações lineares dos vetores $e_{m}$.

Da relação de comutação segue que $\mathrm{H}^{\mathrm{n}-\mathrm{r}}$ localiza-se no centro de 
$\alpha(\alpha)$ e que $H^{r} \oplus K^{\nu}$ é um ideal isomörfo a $b$.

\section{Teorema A:}

A àlgebra de Lie quasehomogēnea $\propto(\alpha)$ é determinada, a menos de um número finito de variantes, por seu sistema de raí zes (como um subespaço do espaço gerado pelas raízes) e por sua dimensão.

\section{Demonstração:}

As relações (1)-(4) do Lema 1.1 expressam os comutado res embe a geometria das raizes, sem referência as coordenadas (excluindo a escolha de sinal tem uma das relações (3)). As sim, o conjunto de raizes, os vetores no $\mathbb{C}$-espaço vetorial $\mathbb{C}^{\mathbf{r}}$, determina $b$ menos de um número finito de possibilidades.

\section{Teorema B :}

Assumimos que o suporte é completo. Então a ação da älgebra de Lie $\not(\alpha)$ no espaço das funções no suporte é univocamente determinada em relação a classe de equivalência afim dos pares (suporte, sistema de raizes).

\section{Demonstração:}

Denotamos. por $\sigma_{m}$ a ação de translação para $m \in \mathbb{Z}^{n}$ $\left(\sigma_{m}^{a}\right)(k)=a(k-m)$, onde $k \in \mathbb{Z}^{n}$ e $a: z^{n} \rightarrow \mathbb{C}$. Então:

$e_{m}=\sigma_{m} h^{\prime}$ (este $h^{\prime}$ é o operador de multiplicaço pela $i$-êsima coordenada; $h^{\prime}=x_{i}{ }^{\partial}$ )

De fato: Consideremos o monómio $a=2 x_{1}{ }^{\beta_{1}} \ldots x_{n}{ }^{\beta_{n}}$

$$
k=\left(\beta_{1}, \ldots, \beta_{n}\right)
$$

$a(k)=z \cdot$ Seja $m=\left(m_{1}, \ldots,-1, \ldots, m_{n}\right)$ uma raiz para um certo peso $\left(\alpha_{1}, \ldots, \alpha_{n}\right)$ fixo. 


$$
\begin{aligned}
& h_{i} a=\left(x_{i}{ }^{2}{ }_{i}\right) a=z \beta_{i} x_{1}^{\beta}{ }^{\beta} \ldots x_{n}^{\beta} n \text {. Como operador } \\
& \left(h_{i} a\right)(k)=h_{i}(k) a(k)=B_{i} z \\
& e_{m}=x_{1} m_{1}^{1} \ldots x_{i-1}^{m_{i}-1} x_{i+1}^{m_{i+1}} \ldots x_{n} m_{n} \partial_{i} ; k^{\prime}=\left(\beta_{1}+m_{1}, \ldots, \beta_{n}+m_{n}\right) \\
& e_{m} a=z \beta{ }_{i} x_{1}^{\beta}{ }^{+} m_{1} \ldots x_{i}^{\beta}{ }^{\beta}-1 \ldots x_{n}^{\beta}{ }^{\beta}+m_{n} \\
& \text { (*) }\left(e_{m}^{a}\right)\left(k^{\prime}\right)=\beta_{i}{ }^{z} \\
& \left(\sigma_{m}\left(h_{i}{ }^{a}\right)\right)\left(k^{\prime}\right)=\left(h_{i} a\right)(k)=\beta_{i}{ }^{2} \text { de }(*) \text {. Portanto } \\
& \mathrm{e}_{\mathrm{m}}=\sigma_{\mathrm{m}} \mathrm{h}_{\mathrm{i}}
\end{aligned}
$$

Estendemos as funçóes no suporte sobre o reticulado $\mathbb{Z}^{n-1}$ de todos os pontos inteiros no plano suporte, fazendo tudo igual a zero fora do suporte. Os operadores $h_{i}, e_{m}, \sigma_{m}, h_{m}$ defini dos anteriormente nas funçōes em $\mathbb{Z}^{\text {n }}$ também atuam no espaço das fun cões nos reticulados $\mathbb{Z}^{\text {n-1 }}$ no plano suporte. Estes operadores no espaco das funcões em $\mathbb{Z}^{n-1}$ são denotados pelas mesmas letras. As sim $h_{i} e h_{m}$ são operadores de multiplicação pelas funcões afins no plano suporte, $\sigma_{m} e ́$ o operador de translação pela raiz m e $e_{m}=\sigma_{m} h_{i}$.

Suponha que mé uma raíz. Chamamos um ponto k do supor te um ponto base para $\underline{\text { m }}$ se $k+m$ não pertence ao suporte. o conjun to de todos os pontos bases de mé chamado a base da raiz m (no dado suporte).

A base da raiz $m=p^{-1}{ }_{i}$ é formada por todos os pontos do suporte cuja i-ésima coordenada é 0 . Consequentemente a base inteira localiza-se no hiperplano afim $\mathbb{C}^{n-2}$ do plano suporte.

A base de cada raiz do suporte completo pertence a pre cisamente um hiperplano afim $\mathbb{C}^{n-2} \subset \mathbb{C}^{n-1}$. Cada ponto do suporte $e^{-}$ obtido de um ponto da base por subtracão de um múltiplo inteiro 
não negativo m, assim se a base está contida em $c^{n-3}$, o suporte estará contido em $\mathbb{C}^{n-2}$ e não será completo.

Assim, existe precisamente uma funçào afim no plano suporte que é igual a 0 na base de m e cujo incremento ao longo

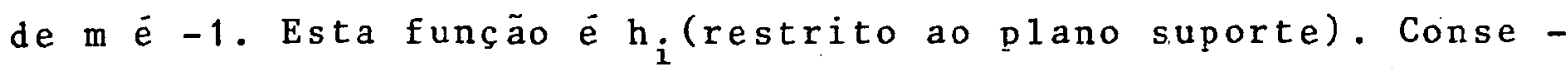
quentemente, a restrição de $h_{i}$ ao plano suporte, pode ser reconstruido univocamente do suporte e a raiz m.

A ação de e no espaço das funçóes no suporte pode agora ser descrito em termos somente da geometria do suporte e as raizes: $e_{m}=\sigma_{m} h_{i}$, isto $\dot{e},\left(e_{m} a\right)(k)=h_{i}(k-m) a(k-m) \quad$ para qualquer função a. Notamos que o operador $e_{m}$ deixa o espaço das funções invariantes e que anula o exterior do suporte desde que $h_{i}$ anula os pontos base.

A àlgebra $(\alpha)$ atua no espaço das funções num reticulado do plano suporte, assim temos a representação $\phi: \pi(\alpha) \rightarrow \xi$, onde $\xi$ é a àlgebra de endomorfismo deste espaço de funções (de dimensão infinita). Consideremos a imagem da representação $\phi$. Es ta imagem é determinada pela geometria do suporte a pelas raízes. Mais precisamente, seja $x_{1}=x\left(\alpha^{1}\right)$ e $\alpha_{2}=\not\left(\alpha^{2}\right)$ duas älgebras quasehomogēnneas e $s_{1} \subset \mathbb{C}_{1}^{n-1}, s_{2} \subset \mathbb{C}_{2}^{n-1}$ suportes completos. Se ja $\psi: c_{1}^{n-1} \rightarrow c_{2}^{n-1}$ uma aplicação afim que leva $s_{1}$ em $s_{2}$ bijetivamente e o sistema de raizes de $\dot{x}_{1}$ no de $\mathfrak{k}_{2}$. Então o isomorfís mo $\psi^{*}$, induzido por $\psi$, do espaço de funções em $\mathbb{C}_{2}^{n-1}$ no espaço de funções em $\mathbb{C}_{1}^{n-1}$ leva a älgebra de Lie $\phi\left(\alpha_{2}\right)$ isomorficamente na àlgebra de Lie $\phi\left(\alpha_{1}\right)$.

A $x(\alpha)$ é gerada sobre $C$ e pelos monómios $x_{i}{ }^{2}$ e $x^{m} x_{i}{ }^{\partial}(m) \cdot A$ imagem dos campos $\sum c_{i} x_{i}{ }^{\partial}$ em $\varepsilon$ são operafores 
de multiplicacão por todas as possíveis funcões afins do plano suporte. A imagem do campo $x^{m} x_{i}{ }_{i}(m)$ são os operadores $e_{m}$, def $\underline{i}$ nidos pela geometria do suporte e as.raizes. Consequentemente, $\psi^{*} \cdot e_{\phi(m)}=e_{m} \psi^{*}$ e assim, $\psi$ induz um isomorfismo $\psi: \phi\left(x_{1}\right) \rightarrow \phi\left(x_{2}\right)$ 0 núcleo do homomorfismo de älgebras de Lie $\phi: x(\alpha) \rightarrow \boldsymbol{\varepsilon}$

é 0 . De fato:

$$
\begin{aligned}
& \left(\mathrm{h}+\sum \mathrm{c}_{\mathrm{m}} \mathrm{e}_{\mathrm{m}}\right)(\mathrm{a})=0 \quad \forall \mathrm{a} \\
& \text { Podemos tomar } \delta_{k} \text { que é igual a l somente em } k \text {. } \\
& \left(h+\sum c_{m} e_{m}\right)\left(\delta_{k}\right)(p)=h(p) \delta_{k}(p)+\sum c_{m} h i(m) \delta_{k}(p-m) \\
& \text { II } \\
& \left(h(k) \delta_{k}\right)(p) \text { se } p=k+m \text { obtemos } \\
& \sum_{c_{m}} h_{i}(k) \delta_{k+m}
\end{aligned}
$$

Para um $k$ qualquer

$$
\begin{aligned}
& \left(h+\sum c_{m} e_{m}\right)\left(\delta_{k}\right)(k)=h(k)=0 \Rightarrow h=0 \\
& \left(\sum c_{m} e_{m}\right)\left(\delta_{k-\bar{m}}\right)(k)=\sum c_{m} h_{i(m)}(k) \delta_{k-m}(k-m)= \\
& =c_{\bar{m}} h_{i(m)}(k) \text {. Escolhemos k tal que } h_{i(\bar{m})}(k) \neq 0 \Rightarrow \\
& \Rightarrow c_{\bar{m}}=0 .
\end{aligned}
$$

Assim a älgebra de Lie $\phi(\not(\alpha))$ é isomorfa a $\not(\alpha)$. 0 isomorfismo $a_{2} \rightarrow \phi\left(\alpha_{2}\right) \stackrel{\Psi}{\rightarrow} \phi\left(x_{1}\right) \rightarrow w_{1}$ mostra que ambas as älgebras $x_{1}$ e $x_{2}$ e suas acões no espaco de funções em $s_{1}$ e $\mathrm{S}_{2}$ são isomorfas.

\section{Corolärio 1:}

Suponha que o conjunto de pesos a e o grau d são tais que existe uma função quasehomogênea com um ponto crítico isola do 0 com 2-jato nulo. Então o sistema de raízes e o suporte de termina univocamente a älgebra de Lie $x(\alpha)$ e sua ação $\phi$. 


\section{Corolário 2:}

Seja $\gamma: \mathbb{C}_{1}^{n-1} \rightarrow \mathbb{C}_{2}^{n-1}$ um isomorfismo afim de um suporte completo plano $\mathrm{s}_{1}$ em $u m$ suporte completo $\mathrm{plano}_{2}$, levando $\mathrm{s}_{1}$ em parte de $s_{2}$, as raizes de $a_{1}$ em parte das raizes de $a_{2}$ as bases das raizes de $x_{1}$ nas parte das bases das correspondentes raí zes de $x_{2}$. Então $\gamma$ induz um isomorfismo entre a ação $\phi$ de $x_{1}$ no espaço de funções em $\mathrm{S}_{1}$ e a ação de uma subálgebra de $\mathbb{K}_{2}$ no espaço de funções em $S_{2}$ que se anul a fora de $\gamma\left(S_{1}\right)$.

\section{Corolärio 3 :}

Suponhamos que sob as hipóteses do Corolärio 2, värios pontos do suporte $S_{1}$ são distinguidos e os valores das funções são fixados para eles. Todas as funções em $\mathrm{s}_{1} \mathrm{com}$ valores fixados por estes pontos formam um plano afim $p$ no espaço de fun ções $S_{1}$. Seja $a_{p}$ álgebra de isotropia deste plano afim. Então o isomorfismo $\gamma$ do Corolário 2 induz um isomorfismo da ação de $\mathfrak{x}_{p}$ em $\mathrm{P}$ com a ação de a lguma subālgebra da àlgebra de Lie $\varkappa_{2}$, pre servando o plano $\gamma^{-1 *} \mathrm{P}$ no espaço de funções em $\mathrm{S}_{2}$.

Nas demonstrações dos teoremas 1.6 e 1.11 utilizamos os resultados obtidos acima.

\section{Teorema $1.6\left(10_{k}\right)$ :}

$$
\begin{aligned}
j^{3}, y^{3 k-1} f & =x^{3} \Rightarrow \text { um dos três caso ocorrem: } \\
j_{x^{3}, y^{3 k}} & \approx x^{3}+a x^{2} y^{k}+y^{3 k}, 4 a^{3}+27 \neq 0 \\
& \approx x^{3}+x^{2} y^{k} \\
& \approx x^{3}
\end{aligned}
$$


Vamos utilizar o Corolário 3

Se $\mathrm{j}_{\mathrm{x}}{ }^{3}, \mathrm{y}^{3 k-1} \mathrm{f}=\mathrm{x}^{3} \Rightarrow \mathrm{f}=\mathrm{x}^{3}+a \mathrm{x}^{2} \mathrm{y}^{\mathrm{k}}+\mathrm{bxy} \mathrm{y}^{2 k}+c \mathrm{y}^{3 k}$

peso: $\alpha=(k, 1)$ e grau: $d=3 k$

suporte: $\quad s_{1}=(3,0) ; \quad s_{2}=(2, k) ; \quad s_{3}=(1,2 k) ; \quad s_{4}=(0,3 k)$. Logo os monômios bases são: $x^{3}, x^{2} y^{k}, x y^{2 k}, y^{3 k}$.

raizes: $m=(-1, k)$, temos então: $x_{x}=h_{1} e e_{m}=x^{-1} y^{k} x_{x}=y^{k} x_{x}$ ponto base: $(0,3 \mathrm{k})$

Seja $\gamma: \mathbb{C}_{1} \rightarrow \mathbb{C}_{2}$ descrita no Corolário.2, dada por

$\gamma(r, s)=(r, s / k)$

Seja $S_{1}$ o suporte acima e $S_{2}$ dado por $(3,0) ;(2,1)$;

$(1,2) ;(0,3)$. Logo os monomios bases são: $\mathrm{X}^{3}, \mathrm{X}^{2} \mathrm{Y}, \mathrm{XY} \mathrm{Y}^{2}, \mathrm{Y}^{3}$.

observamos que temos $(-1,1)$ e $(1,-1)$ como raízes, mas tomaremos a subälgebra de Lie correspondente a raíz $(-1, k)$ e portanto tomaremos como raiz $\mathrm{m}^{\prime}=(-1,1)$ que tem como ponto base $(0,3)$.

Devemos mostrar que $\gamma^{*} \mathrm{e}_{\gamma(\mathrm{m})}=\mathrm{e}_{\mathrm{m}} \gamma^{*}$

De fato: $\gamma^{*}: \mathcal{F}_{2} \rightarrow \mathcal{F}_{1}\left(\mathcal{F}_{1}\right.$ e $\mathcal{F}_{2}$ são espaços de funções de $V^{n-1}$

$$
\begin{gathered}
\text { em } c) \\
\text { Seja } \alpha \in \mathcal{f}_{2} ; \gamma^{*} \alpha=\alpha_{0} \gamma \\
\left(e_{m} \gamma^{*}\right)(\alpha)(t)=\left(e_{m}\left(\alpha_{0} \gamma\right)(t)=x_{x}(t-m)\left(\alpha_{0} \gamma\right)(t-m)\right. \\
\text { os t's que nos servem serão: } \\
t_{1}=(2, k) \text { pois } t_{1}-m=(3,0) \Rightarrow 3 \alpha(3,0) \\
t_{2}=(1,2 k) \text { pois } t_{2}-m=(2, k) \Rightarrow 2 \alpha(2,1) \\
t_{3}=(0,3 k) \text { pois } t_{3}-m=(1,2 k) \Rightarrow 1 \alpha(1,2) \\
t_{4}=(-1,4 k) \text { pois } t_{4}-m=(0,3 k) \Rightarrow 0 \alpha(0,3) \\
\left(\gamma^{*} e_{m} \cdot(\alpha)\right)(t)=e_{m} \cdot(\alpha){ }_{0}(\gamma(t))=x_{x}\left(\gamma(t)-m^{\prime}\right) \alpha\left(\gamma(t)-m^{\prime}\right)
\end{gathered}
$$




$$
\begin{gathered}
\gamma\left(t_{1}\right)=(2,1) \Rightarrow \gamma\left(t_{1}\right)-m^{\prime}=(3,0) \Rightarrow 3 \alpha(3,0) \\
\gamma\left(t_{2}\right)=(1,2) \Rightarrow \gamma\left(t_{2}\right)-m^{\prime}=(2,1) \Rightarrow 2 \alpha(2,1) \\
\gamma\left(t_{3}\right)=(0,3) \Rightarrow \gamma\left(t_{3}\right)-m^{\prime}=(1,2) \Rightarrow 1 \alpha(1,2) \\
\gamma\left(t_{4}\right)=(-1,4) \Rightarrow \gamma\left(t_{4}\right)-m^{\prime}=(0,3) \Rightarrow 0 \alpha(0,3) \\
\text { Logo } e_{m} \gamma^{*}=\gamma^{*}{ }^{e} \gamma(m)
\end{gathered}
$$

Como as hipóteses do corolärio 3 estão satisfeitas, en tão o problema reduz-se à classificação dos polinômios $P$ : $\mathrm{x}^{3}+$ $+A X^{2} Y+B X Y^{2}+C Y^{3}$. Como $e_{m}=y_{X}$, o deslocamento na direção de $x$ deve ser $\beta y$, isto é:

$$
\left\{\begin{array}{l}
X=\alpha x+\beta y \\
Y=\delta y
\end{array}\right.
$$

is to é, $\alpha^{3} x^{3}+\left(3 \alpha^{2} \beta+A \alpha^{2} \delta\right) x^{2} y+\left(3 \alpha \beta^{2}+2 A \alpha \beta \delta+B \alpha \delta^{2}\right) x y^{2}+\left(\beta^{3}+A \beta^{2} \delta+\right.$ $\left.+B \beta \delta^{2}+C \delta^{3}\right) y^{3}=x^{3}+a x^{2} y+y^{3}$ para $\alpha, \beta, \delta$ convenientes.

Fazendo $x=X / Y$ em $\frac{X^{3}}{Y^{3}}+\frac{a X^{2} Y}{y^{3}}+\frac{Y^{3}}{y^{3}}$, obtemos $x^{3}+a x^{2}+1$

Segue da solução de Cardan para a equação cúbica que para existirem 3 (três) raízes distintas, devemos ter $4 a^{3}+27 \neq 0$.

Caso as raizes tenham multiplicidade 2 ou 1 , entäo podemos fazer uma substituição $x=x+\lambda y$ e $P$ passa a ter a for ma $\mathrm{X}^{3}+A \mathrm{X}^{2} \mathrm{Y}, \mathrm{A} \neq 0$. Dilatando $\mathrm{Y}$ obtemos $\mathrm{X}^{3}+\mathrm{X}^{2} \mathrm{Y}$. Se a multiplici dade é 3 (três), então $P=X^{3}$ e assimo teorema 1.6 fica demonstra do.

Veremos no Capitulo II que esta classificaça de sin gularidades do teorema 1.6 coincide com o problema da classifica ção afim de triplas de pontos em $\mathbb{C}^{1}$. 
$j_{x^{3}, y^{3 k^{f}}=x^{3}+a x^{2} y^{k}+y^{3 k}, 4 a^{3}+27 \neq 0 \Rightarrow f \in J_{k, 0}}$.

Forma normal de $J_{k, 0}: x^{3}+a x^{2} y^{k}+y^{3 k}+c x y^{2 k+1}$, onde $c=c_{e^{+}} \ldots+c_{k-3} y^{k-3}$ para $k>2$, para $k=2$, tomamos $c=0$.

\section{Demonseração}

Mostraremos que $\mathrm{f}_{\mathrm{o}}=\mathrm{x}^{3}+a x^{2} \mathrm{y}^{\mathrm{k}}+\mathrm{y}^{3 \mathrm{k}} \overline{\mathrm{e}}(4 \mathrm{k}-1)$-determinado.

$$
\begin{aligned}
\frac{\partial f_{o}}{\partial x}= & 3 x^{2}+2 a x y^{k} \quad \text { e } \frac{\partial f o}{\partial y}=a k x^{2} y^{k-1}+3 k y^{3 k-1} \\
y^{4 k}= & \frac{1}{3 k} y^{k+1}\left(a k x^{2} y^{k-1}+3 k y^{3 k-1}\right)-\frac{1}{6} x y^{k}\left(3 x^{2}+2 a x y^{k}\right)+ \\
& +\frac{1}{2 k a} x y\left(a k x^{2} y^{k-1}+3 k y 3 k-1\right)-\frac{3}{4 a^{2}} y^{2 k}\left(3 x^{2}+2 a x y^{k}\right)+ \\
& +\frac{9}{4 a^{3} k} y^{k+1}\left(a k x^{2} y^{k-1}+3 k y^{3 k-1}\right)-\frac{27}{4 a^{3}} y^{4 k}
\end{aligned}
$$

Logo:

$$
\begin{aligned}
\left(1+\frac{27}{4 a^{3}} y^{4 k}=\right. & \frac{1}{3 k} y^{k+1 \partial f_{o}}-\frac{1}{\partial y} x y^{k \partial f_{o}} \frac{1}{\partial x}+\frac{1}{2 k a} x \frac{\partial f_{o}}{\partial y}- \\
& -\frac{3}{4 a^{2}} y^{2 k} \frac{\partial f_{o}}{\partial x}+\frac{9}{4 a^{2} k} y^{k+1 \frac{\partial f_{o}}{\partial y}}
\end{aligned}
$$

Para ser determinado, ou seja não degenerado $27+4 a^{3} \neq 0$

$$
\text { Então: } \begin{aligned}
y^{4 k}= & \frac{4 a^{3}}{3 k\left(27+4 a^{3}\right)} y^{k+1} \partial_{2} f_{0}-\frac{4 a^{3}}{\left(27+4 a^{3}\right) 6} \times y^{k} \partial_{1} f_{o}+ \\
& +\frac{2 a^{2}}{k\left(27+4 a^{3}\right)} x^{y \partial}{ }_{2}{ }_{0}-\frac{3 a}{\left(27+4 a^{3}\right)} y^{2 k} \partial_{1} f_{o}+ \\
& +\frac{9}{k\left(27+4 a^{3}\right)} y^{k+1} \partial{ }_{2} f_{0} . \\
x^{2} \phi & =\frac{1}{3}{ }_{1} f_{0} \phi+m^{4 k}, \text { onde } \phi \bar{e} \text { um monômio em } x \text { e y com grau } \geq \\
& \geq 2 k
\end{aligned}
$$


nea com parte quasehomogēnea fo é equivalente a uma funça da forma $f_{o}+\sum c_{k} e_{k}$, onde $c_{k}$ são constantes e $e_{1}, e_{2}, \ldots, e_{s} e ́$ o sis tema de monomios superiores da base em uma base fixa do anel 1o cal da função $f_{0}$ :

os monômios acima da diagonal são de grau superior a $3 k$ ou seja maior ou igual a $3 k+1$.

$$
\begin{aligned}
& x^{p}: k p>3 k+1 \text { está contido no ideal. } \\
& y^{3 k+1} \text { : està contido no ideal. } \\
& \text { Restam elementos da forma } x y \\
& \text { Queremos } k+p \geqq 3 k+1 \Rightarrow p \geqq 2 k+1 \\
& \frac{d}{\text { max }}_{N}=n-2 \sum \alpha_{i}=2-2\left(\frac{k}{3 k}+\frac{1}{3 k}\right)=\frac{6 k-2 k-2}{3 k}=\frac{4 k-2}{3 k}
\end{aligned}
$$

Logo $k+p$ é no máximo $4 k-2 \Rightarrow p=3 k-2$.

Assim $f=x^{3}+a x^{2} y^{k}+y^{3 k}+c x y^{2 k+1}$, com $c$ dado acima.

No capitulo II faremos dois tipos de estudos com a

forma normal obtida no teorema 1.7. 0 primeiro é encontrar os possiveis elementos de uma mesma órbita após a ação de difeomor fismos em $f_{0}$, em seguida passando ao quociente pela relação"ger mes de uma mesma örbita", encontraremos o espaço de órbitas. 0 segundo é de como se comporta $f^{\prime}$ quando aplicamos estes mesmos difeomorfismos.

Para emonstrar o próximo teorema (teorema 1.8) utili zaremos uma técnica que descreveremos a seguir denominada "solu cão das palavras cruzadas". Esta técnica que auxilia na classificação de singularidades de:germes em duas variáveis, constitui-se em certas construções geométricas que descrevermos a seguir.

Suponha que cada derivada parcial da funça dada não contenha mais que dois monomios. No plano dos expoentes junta - 
mos os monômios (expoentes) da derivada parcial em relação a $x$ por um segmento que chamamos -x-segmento fundamental. Analogamente, segmentos fundamentais são definidos para as outras va riäveis.

Os segmentos fundamentais descrevem afinidades entre as imagens de seus monómios no anel local. Consideremos algumas consequências destas afinidades. Chamamos toda translação de um segmento fundamental por um vetor inteiro com componentes não negativas de segmento permitido. Notamos que dois segmentos per mitidos podem localizar-se um sobre o outro e coincidir geome tricamente(se um é um $x$-segmento e o outro um y-segmento).

Dois segmentos permitidos são ditos ligados se eles tem um final comum. Uma cadeia permitida é uma coleção de seg mentos permitidos tal que cada um dos segmentos é ligado um ao outro numa sequência de segmentos consecutivos ligados na coleção. Uma cadeia ê dita maximal se ela não è parte de nenhuma cadeia permitida maior.

Um ciclo é uma sequência consecutiva de segmentos per mitidos ligados a qual o último segmento è ligado ao primeiro. Um ciclo é dito ser regular se ao longo de seu caminho cada direção $x-(y)-\ldots$-segmentos é percorrido o mesmo nûmero de vezes como os outros.

Podemos formulàr agora a regra "solução das palavas cruzadas" para uma função f com um ponto critico de multiplicidade finita: 
(b) Se todos os ciclos são regulares, então a dimensão $\mu$ do anel local é igual ao número de ciclos maximais. Neste caso podemos achar uma base do anel local tomando arbitrariamente um mono mio de cada uma das cadeias maximais.

(c) Al'en d'sso, dada una fíltraçao, obtemos uma base regular escolhendo um monómio de filtração maior de cada uma das cadeias maximais.

$$
\frac{\text { Teorema } 1.8(12 k):}{j_{x, y} 3 k f=x^{3}+x^{2} y^{k} \Rightarrow f \in J_{k, p}(p>0)}
$$

Forma normal de $J_{k, p}: x^{3}+x^{2} y^{k}+a y^{3 k+p}(k>1, p>0)$, onde: $a=a_{0}+\ldots+a_{k-2} y^{k-2}\left(a_{0} \neq 0\right)$.

\section{Demonstração:}

Suponhamos que f se escreve como:

$$
\begin{array}{r}
x^{3}+x^{2} y^{k}+x \phi(y)+\psi(y), \text { onde } \phi(y) \in k^{2 k+1} \text { e } \\
\psi(y) \in k^{3 k+1}
\end{array}
$$

Queremos efetuar uma mudança de coordenadas de tal for ma que o termo $x \phi(y)$ desapareça.

$$
\begin{aligned}
\text { Façamos a mudança: }\left\{\begin{aligned}
& \mathrm{x}=\mathrm{X}+\beta(\mathrm{y}) \\
& \mathrm{y}=\mathrm{Y}
\end{aligned}\right. \\
\text { Ficamos com: } \mathrm{X}^{3}+3 \mathrm{X}^{2} \beta(\mathrm{y})+3 X \beta^{2}(\mathrm{y})+\beta^{3}(\mathrm{y})+ \\
+\mathrm{X}^{2} \mathrm{Y}^{\mathrm{k}}+2 \mathrm{X} \beta(\mathrm{y}) \mathrm{Y}^{\mathrm{k}}+\beta^{2}(\mathrm{y}) \mathrm{Y}^{\mathrm{k}}+ \\
+\mathrm{X} \phi(\mathrm{y})+\beta(\mathrm{y}) \phi(\mathrm{y})+ \\
+\psi(\mathrm{y}) .
\end{aligned}
$$




$$
\begin{aligned}
& \text { Assim } B(y)=\left(-2 y^{k} \pm \sqrt{4 y^{2 k}(1-3 \theta(y))}\right) / 6 \\
& \text { Tomamos } \beta_{1}(y)=\left(-2 y^{k}+2 y^{k} \sqrt{1-3 \theta(y)}\right) / 6= \\
&=y^{k}[(-1+\sqrt{1-3 \theta(y))} / 3] \text { que é uma função }
\end{aligned}
$$

analitica, $\operatorname{com} \beta_{1}(y) \in \mathbb{H}^{\mathrm{k}+1}$.

Fazendo então a mudança: $\left\{\begin{array}{l}x=X+\beta_{1}(y) \text { obtemos: } \\ y=Y\end{array}\right.$

(*) $X^{3}+X^{2}\left(Y^{k}+3 \beta_{1}(y)\right)+\sigma(y)$, onde $\sigma(y)=\beta^{3}(y)+\beta^{2}(y) Y^{k}+$

$+\beta(y) \phi(y)+\psi(y) \in \nVdash^{3 k+1}$, Dois $\beta^{3}(y) \in \nVdash^{3 k+3}, \beta^{2}(y) \in \nVdash^{3 k+2}$,

$B(y) \phi(y) \in \nVdash^{3 k+2}$ e $\psi(y) \in \mathbb{H}^{3 k+1}$.

chamamos o coeficiente do termo de maior grau de $\tau(n)$ de $a_{0}$.

$$
\text { Observamos que: } y^{k}+3 \beta_{1}(y)=y^{k}(1+\gamma(y)) \text {, com }
$$

$\gamma(y) \in$ the

Fazendo a mudança: $n=y \sqrt[k]{1+\gamma(y)}$ e $\dot{\xi}=x$ em $(*)$, obte mos:

$$
\begin{aligned}
& \xi^{3}+\xi^{2} n+\tau(n) \text { onde } n \epsilon m^{3 k+1} \\
& \text { Como } f_{o} \text { é dado por } x^{3}+x^{2} y^{k}+a_{o} y^{3 k+p} \text {, vamos utilizar }
\end{aligned}
$$

a "solução de palavras cruzadas" para verificar até que grau temos os termos em y.

Os segmentos fundamentais são obtidos através das der vadas parciais em relação a $x$ e a $y$.

$$
\frac{\partial f_{o}}{\partial x}=3 x^{2}+2 x y^{k} \quad e \frac{\partial f_{o}}{\partial y}=k x^{2} y^{k-1}+(3 k+p) a_{o} y^{3 k+p-1}
$$

Queremos encontrar o maior grau q de tal forma que o expoente do monómio $y^{3 k+p+q}$ não pertença a uma cadeia permitida infinita (pois neste caso ele pertenceria ao ideal gerado pela derivada parcial). Consideremos então as cadeias de segmentos per mitidos: 


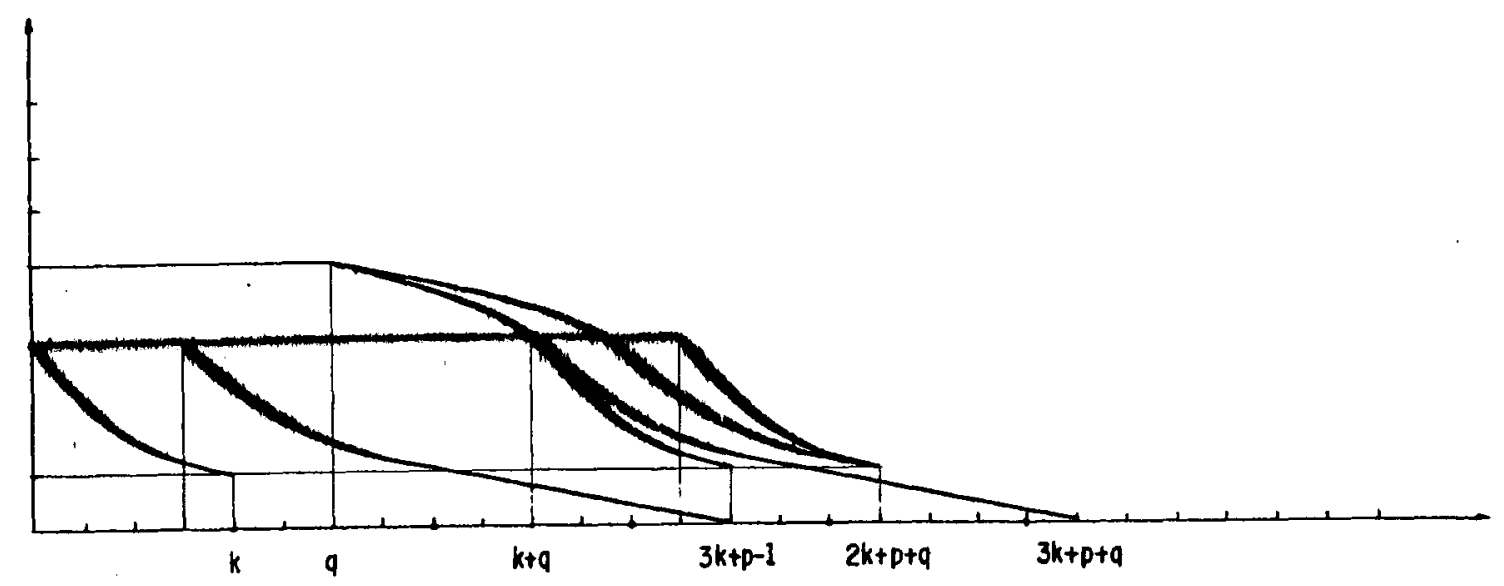

$y^{3 k+p+q} \rightarrow x^{2} y^{k+q}<x^{3} y^{q} \quad$ quando $q \geqq k-1$

As restrições (1) e (2) são para que as translações dos segmentos fundannentais sejan feitas por vetores inteiros com compontes nao negativas.

Escolhemos então o caso (2). Logo

$x^{3} y^{q} \rightarrow x y^{2 k+p+q} \rightarrow x^{2} y^{k+p+q} \rightarrow y^{(3 k+p+q)+1}$

Assim os monómios $\left(y^{q} \cdot y^{3 k+p}\right)$ onde $q<k-1$ não estão no ideal.

Portanto pela "soluçăa de paiavras cruzaráas" nosso $f$ sera da forma $x^{3}+x^{2} y^{k}+a y^{3 k+p}$, onde $a=a_{0}+\ldots+a_{k-2} y^{k-2}(a \neq 0)$.

Para a demonstração do pröximo teorema necessitamos do seguinte J. ma :

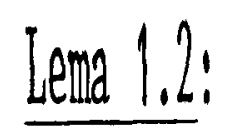

Toda forma binäria não-degenerada de grau $\geqq 4$ reduz-se à forma nor mal com (n-3)-parāmetros:

$$
x^{n-1} y+c_{1} x^{n-2} y^{2}+\ldots+c_{n-3} x^{2} y^{n-2}+x y^{n-1} .
$$


Teorema 1.9 (13):

$$
\begin{aligned}
j_{3} f(x, y)=0 \Rightarrow \text { ocorre um dos seis casos: } & \\
j_{4} & \approx x^{4}+a x^{2} y^{2}+y^{4}, a^{2} \neq 4 \\
& \approx x^{4}+x^{2} y^{2} \\
& \approx x^{2} y^{2} \\
& \approx x^{3} y \\
& \approx x^{4} \\
& \approx 0
\end{aligned}
$$

Demonstração:

$$
f=\left(x-\beta_{1} y\right)\left(x-\beta_{2} y\right)\left(x-\beta_{3} y\right)\left(x-\beta_{4} y\right)
$$

10. Caso: $\beta_{1}=\beta_{2}=\beta_{3}=\beta_{4} \Rightarrow j_{4} f \approx x^{4}$.

20. Caso: $\beta_{1}=\beta_{2}=\beta_{3} \neq \beta_{4} \Rightarrow j_{4} f \approx x^{3} y$.

30. Caso: $\beta_{1}=\beta_{2} \neq \beta_{3}=\beta_{4} \Rightarrow j_{4} f \approx x^{2} y^{2}$.

40 Caso: $\beta_{1}=\beta_{2} \not \beta_{3} \quad ; \beta_{1} \neq \beta_{4}$ e $\beta_{3} \neq \beta_{4} \Rightarrow j_{4} f \approx x^{2}\left(x^{2}+y^{2}\right)=x^{4}+x^{2} y^{2}$.

50 Caso: $\beta_{i} \neq \beta_{j} \quad i \neq j, i, j=1,2,3$ ou 4

Segue do lema 1.2 que $x^{4}+a_{1} x^{3} y+a_{2} x^{2} y^{2}+a_{3} x y^{3}+y^{4}$ reduz-se $a$ $x y\left(x^{2}+2 c x y+y^{2}\right)$, isto $\dot{e}$, no produto das formas quadräticas $a=x y$ e $b=x^{2}+2 c x y+$ $+y^{2}$. Existem duas formas degeneradas independentes entre as combinações linea res $p a+q b$ de $a$ e $b\left(p: q\right.$ é definido da equação característica $p^{2}+4 c p q$ + $+4\left(c^{2}-1\right) q^{2}=0$ cujo discriminante è não nulo).

Tomando as raizes destas formas degeneradas como coordenadas, reduzimos a forma original a $c_{1} x^{4}+c_{2} x^{2} y^{2}+c_{3} y^{4}$. A condição para tal forma ser não-degenerada é que $c_{1} c_{3}\left(4 c_{1} c_{3}-c_{2}^{2}\right) \neq 0$. Por uma dilatação nas coordena- 
$j_{4} f=x^{4}+a x^{2} y^{2}+y^{4}, a^{2} \neq 4 \Rightarrow f \in x_{9}$

A forma normal de $x_{0}$ e: $x^{4}+a x^{1} y^{1}+y^{4}$, com $a^{2} \neq 4$.

Demonstração:

Basta mostrar a suficiencia do jato de $f_{0}: x^{4}+a x^{2} y^{2}+y^{4}$, para tanto exprimimos $y^{5}, y^{4} x, y^{3} x^{2}, y^{2} x^{3}, y x^{4}$ e $x^{5}$ em termos de $\partial_{1} f_{0}$ e $\partial_{2} f_{0}$.

$$
\partial_{1} f_{0}=4 x^{3}+2 a x y^{2} \text { e } \partial_{2} f_{0}=2 a x^{2} y+4 y^{3}
$$


que é uma função racional de grau 3 em $\lambda$.

Consequentemente se as raîzes de $D^{\prime}$ são simples, então no mínimo uma das raízes de $\lambda$ não faz o número $a b-9$ nulo.

Concluímos' então que no caso de raizes simples, P reduz-se à forma $x^{4}+b x^{3} y+a x^{2} y^{2}+x y^{3}$, onde $\Delta=4\left(a^{3}+b^{3}\right)+27 \neq a^{2} b^{2}+18 a b$ e ab $\neq 9$

No caso de uma raiz de multiplicidade dois, podemos levar $\mathrm{P}(\mathrm{x}, \mathrm{y})$ à forma $x^{4}+A^{\prime} x^{3} y+B^{\prime} x^{2} y^{2}$ após o qual fazer $y=\delta y^{\prime}$ e obter $x^{2} \cdot\left(x^{2}+c x y+y^{2}\right) \operatorname{com}$ $a^{2} \neq 4$.

\section{$3 y$}

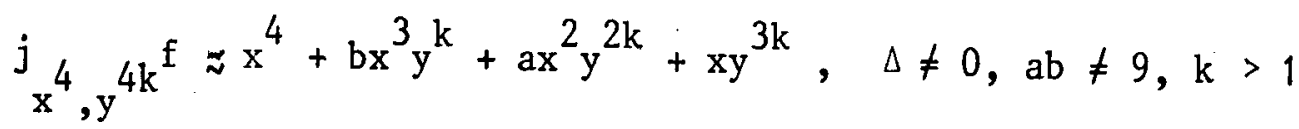

$$
\begin{aligned}
& \approx x^{2}\left(x^{2}+a x y^{k}+y^{2 k}\right), a^{2} \neq 4 \quad k>1 \\
& \approx \mathrm{x}^{2}\left(\mathrm{x}+\mathrm{y}^{\mathrm{k}}\right)^{2} \quad \mathrm{k}>1 \\
& \approx \mathrm{x}^{3}\left(\mathrm{x}+\mathrm{y}^{\mathrm{k}}\right) \quad \mathrm{k}>1 \\
& \approx x^{4} \quad \text { onde } \Delta=4\left(a^{3}+b^{3}\right)+27-a^{2} b^{2}-18 a b
\end{aligned}
$$

$$
f=x^{4}+a x^{3} y^{k}+b x^{2} y^{2 k}+c x y^{3 k}+d y^{4 k}
$$

peso: $(k, 1)$

suporte: $(4,0) ;(3, k) ;(2,2 k) ;(1,3 k) ;(0,4 k)\left(s_{1}\right)$

raiz: $(-1, k)$

Tomamos $\gamma(r, s)=(r, s / k)$

suporte: $(4,0) ;(3,1) ;(2,2) ;(1,3) ;(0,4) \quad\left(s_{2}\right)$

Pelo Corolário 3, o problema reduz-se à classificação dos polinô - 
Vamos chamar de $\nu(d)$ o número de monômios de grau d.

Observemos que parapolinômios de grau $4 \mathrm{k} \leqq \mathrm{d} \leqq 5 \mathrm{k}-1, v(\mathrm{~d})=5$, pois $P(x, y)=\alpha x^{4} y^{i}+\beta x^{3} y^{k+i}+\gamma a x^{2} y^{2 k+i}+\delta x y^{3 k+i}+\varepsilon y^{4 k+i}$, onde $d=4 k+i e i<k$. para $4 \mathrm{k} \leqq \mathrm{d} \leqq 5 \mathrm{k}-2$.

Se existir $A, B, C, D$ e $E$ tal que $P(x, y)=A y^{i} \frac{\partial f_{o}}{\partial x}+B y^{k+i \partial f_{o}} \frac{C y^{i+1 \partial f_{o}}}{\partial x}+D x^{2} y^{2 k+i}+E x^{3} y^{k+i}$, en tão $P(x, y)-D x^{2} y^{2 k+i}-E x^{3} y^{k+i}$ pertence ao ideal e assim é não - degenerada. Mas para que a igualdade seja verificada devemos ter:

$$
\begin{aligned}
4 A & =\alpha \\
3 b A+4 B+b k C+E & =B \\
2 a A+3 b B+2 a k C+D & =\gamma \\
A+2 a B+3 k C & =\delta \\
B & =\varepsilon
\end{aligned}
$$

que tem solụção única, pois

$$
\left|\begin{array}{ccccc}
4 & 0 & 0 & 0 & 0 \\
3 b & 4 & b k & 0 & 1 \\
2 a & 3 b & 2 a k & 1 & 0 \\
1 & 2 a & 3 k & 0 & 0 \\
0 & 1 & 0 & 0 & 0
\end{array}\right|=12 k \neq 0 .
$$

para $d=5 k-1$

Os monômios guias serão:

$y^{k-1} x \partial x ; y^{2 k-1} \partial_{x} ; x \partial_{y} ; y^{k} \partial_{y}$.

Necessitamos neste caso de apenas um monômio base: $x^{3}$

0 determinante será: 


$$
\left|\begin{array}{ccccc}
4 & 0 & k b & 0 & 0 \\
3 b & 4 & 2 k a & k b & 1 \\
2 a & 3 b & 3 k & 2 k a & 0 \\
1 & 2 a & 0 & 3 k & 0 \\
0 & 1 & 0 & 0 & 0
\end{array}\right|=\left|\begin{array}{ccc}
4 & k b & 0 \\
2 a & 3 k & 2 k a \\
1 & 0 & 3 k
\end{array}\right|=-4 k^{2}(a b-9) \neq 0 \text {, pois }
$$

$a b-9 \neq 0$

$$
\text { Jâ para } 5 \mathrm{k} \leqq \mathrm{d} \leqq 6 \mathrm{k}-1, \quad(d)=6
$$

\section{para $5 \mathrm{k} \leqq \mathrm{d} \leqq 6 \mathrm{k}-2$}

Os monômios guias são:

$$
\begin{aligned}
& y^{i} x^{2} \partial x, y^{k+i} x \partial x, y^{2 k+i} \partial x, y^{i+1} \partial_{y}, y^{k+i+1} \cdot \partial y \\
& 0 \text { único monómio base } e \cdot x^{3}
\end{aligned}
$$$$
\left|\begin{array}{cccccc}
4 & 0 & 0 & 0 & 0 & 0 \\
3 \mathrm{~b} & 4 & 0 & \mathrm{~kb} & 0 & 0 \\
2 \mathrm{a} & 3 \mathrm{~b} & 4 & 2 \mathrm{ka} & \mathrm{kb} & 1 \\
1 & 2 \mathrm{a} & 3 \mathrm{~b} & 3 \mathrm{k} & 2 \mathrm{ka} & 0 \\
0 & 1 & 2 \mathrm{a} & 0 & 3 \mathrm{k} & 0 \\
0 & 0 & 1 & 0 & 0 & 0
\end{array}\right|=16 \mathrm{k}^{2}(\mathrm{ab}-9) \neq 0
$$

\section{para $\mathrm{d}=6 \mathrm{k}-1$}

Os monōmios guias são:

$$
\begin{aligned}
& y^{k-1} x^{2} \partial_{x} ; y^{2 k-1} x z ; y^{3 k-1} \partial x ; x^{2} \partial y ; y^{k} x \partial y ; y^{2 k} \partial_{y} \\
& \text { o determinante serā: } \\
& \left|\begin{array}{llllll}
4 & 0 & 0 & 0 & 0 & 0 \\
3 b & 4 & 0 & k b & 0 & 0 \\
2 a & 3 b & 4 & 2 k a & k b & 0 \\
1 & 2 a & 3 b & 3 k & 2 k a & k b \\
0 & 1 & 2 a & 0 & 3 k & 2 k a \\
0 & 0 & 1 & 0 & 0 & 3 k
\end{array}\right|=-64 k^{3} \Delta \text {, onde }
\end{aligned}
$$


$\Delta$ è o discriminante do polinômio $x^{3}+b x^{2}+a x+1, \Delta \neq 0$ pois tem a forma $4\left(a^{3}+b^{3}\right)+27-a^{2} b^{2}-18 a b$.

Assim os monômios guias e os monōmios base geram $c^{\nu(d)}$ e f reduz -

-se à forma normal:

$$
\begin{array}{r}
f_{o}+\sum c_{s} e_{s}=x^{4}+b x^{3} y^{k}+a x^{2} y^{2 k}+x y^{3 k} \text {, onde }: \\
a=a_{0}+\ldots+a_{k-2} y^{k-2}, b=b_{0}+\ldots+b_{2 k-2} y^{2 k-2} .
\end{array}
$$




\section{A GEOMETRIA DE ALGUMAS SINGULARIDADES}

Neste Capitulo trataremos de dois tipos de classificação geométrica. Em primeiro lugar aproveitaremos um exemplo da tabela apresentada no artigo [1], que nos fornece uma série de identificações entre os problemas de classificação de singularidades com problemas geométricos. Esta tabela foi obtida utilizando os dois teoremas gerais sobre funçōes quasehomogêneas (Teo remas A e B) dados no Capítulo I. Em segundo lugar, faremos úm estudo da geo metria da parte principal $f_{o}$ das funções semiquasehomogēneas $f=f_{0}+f^{\prime}$ em alguns exemplos particuläres. Em um deles a função a ser estudada é quasehomogēnea e nos restringiremos portanto a encontrar o espaço de órbitas apōs uma mudança de coordenadas permitida. Jā em outros exemplos procuraremos aprofundar o estudo, examinando o comportamento da parte $f$ ' quando aplicamos difeomorfismos que fixam $f_{0}$ a menos de germes da mesma örbita.

\$1. Problemas de classificação de singularidades identificados com problemas $\underline{\text { geométricos }}$

Neste parágrafo explicitaremos um exemplo da tabela de Arnol'd [1] onde è feita a identificação de um problema de classificação de singularidades com um problema geométrico. Feito isto, obteremos o espaço de órbitas através da identificação. Aqui a clásse de objetos geométricos correspondem a blocos de órbitas.

Jä demonstramos o teorema:
$\mathrm{x}^{3}, \mathrm{y}$
$3 \mathrm{k}-\mathrm{f}$ 


$$
\begin{aligned}
\mathrm{j}^{3}, y^{3 k^{f}} & \approx x^{3}+a x^{2} y^{k}+y^{3 k}, 4 a^{3}+27 \neq 0 \\
& \approx x^{3}+x^{2} y^{k} \\
& \approx 0
\end{aligned}
$$

(Teorema 1.6)

Vimos que o problema se reduz a classificação dos polinômios homogêneos $P: X^{3}+a X^{2} Y+b X Y^{2}+c Y^{3}$. Mostraremos que geometricamente isto corresponde a classificação afim de triplas de pontos em $\mathbb{C}^{1}$.

De fato: fatorando $P: X^{3}+a X^{2} Y+b X Y^{2}+c Y^{3}=$

$=(X-A Y)(X-B Y)(X-C Y)$, associamos a cada polinômio, a tripla (não ordenada) de pontos $\{A, B, C\}$.

A mudança de coordenada $X, Y$ permitida $\left(X=X^{\prime}-\beta Y\right.$ e $\left.\underline{Y}=\delta Y^{\prime}\right)$ cor responde a mudanca da tripla $\{A, B, C\}$ para a tripla $\{\delta A+\lambda, \delta B+\lambda, \delta C+\lambda\}$.

$$
\begin{aligned}
P: & \left(\left(X^{\prime}-\beta Y\right)-A Y\right)\left(\left(X^{\prime}-\beta Y\right)-B Y\right)\left(\left(X^{\prime}-\beta Y\right)-C Y\right)= \\
= & \left(X^{\prime}-(A+\beta) Y\right)\left(X^{\prime}-(B+\beta) Y\right)\left(X^{\prime}-(C+\beta) Y\right)= \\
= & \left(X^{\prime}-(\delta A+\lambda) Y^{\prime}\right)\left(X^{\prime}-(\delta B+\lambda) Y^{\prime}\right)\left(X^{\prime}-(\delta C+\lambda) Y^{\prime}\right)
\end{aligned}
$$

Esta mudança $\{A, B, C\} \rightarrow\{\delta A+\lambda, \delta B+\lambda, \delta C+\lambda\}$ pode ser interpretada como obtida pela transformação afim.

$t=\delta u+\lambda, \delta, \lambda \in \mathbb{C}, \delta \neq 0$ na reta complexa a qual interpretamos como plano de Argand-Gauss.

Pondo $\delta=\rho e^{i \theta}$ podemos decompor $t=\rho e^{i \theta} u+\lambda$ em :rés transforma ções elementares:

$$
p=e^{i \theta} u ; \quad s=\rho p ; \quad t=s+\lambda, \text { ou seja, a transformação afim } \dot{e}
$$

a composição de uma rotação, uma homotetia e uma translação.

Vamos agora determinar um sistema de representantes e analisar a estrutura topológica do espaço quociente ou seja o espaço de órbitas. 
Dados os pontos $A, B$ e $C$ distintos em $\mathbb{C}^{1}$, suponhamos que $A$ seja o vér tice dos dois maiores lados do triāngulo $A B C$, B o outro vértice do maior 1ado, : então $C$ é o vërtice dos dois menores lados, como mostra a figura ao lado.

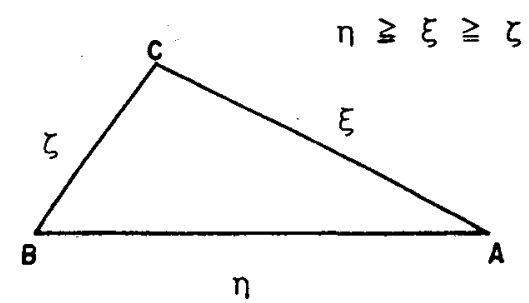

*Observação: A,B e C podem ser colineares, neste caso o resultado continuarä sendo vālido.

Levamos A em $\omega=-1+0 i$ por uma translação, $B$ em $\sigma=1+0 i$ por uma homotetia e uma rotação, então o vértice $C$ é levado na região hachuriada $\Sigma$ na figura abaixo.

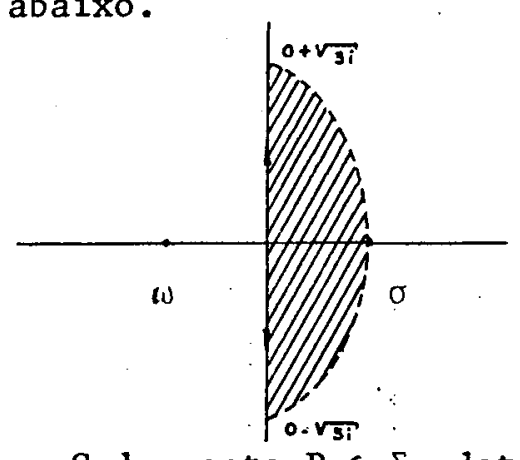

Se $A=B=C$ levamos o ponto em $\omega$

Se $A \neq B=C$ levamos $A$ em $\omega$ e $B$ em $\sigma$.

Cada ponto $P \in \sum$ determina portando uma tripla $\{\omega, \sigma, P\}$ e no caso degenerado $P=\omega$ determina a tripla $\{\omega, \omega, \omega\}$. Vamos identificar $P$ com a tripla correspondente.

Assim temos uma região $\Sigma U\{\omega\}$ que contēm ao menos um representante de cada classe de equivaléncia. Na realidade somente se dois pontos são do contorno da figura, e simétricos em relação ao eixo real eles são equivalen tes, todas as outras classes tem somente um representante em $\Sigma$.

Para obter o espaço de órbitas (ou quociente) observemos portanto que o arco da circunferência no primeiro quadrante deve se identificado com o arco no quarto quadrante, pois os pontos sobre o arco possuem a propriedade que $\overline{\mathrm{AB}}=\overline{\mathrm{AC}}$, então trocando $\mathrm{B}$ por $\mathrm{C}$ justificamos a identificação. Temos ainda que o segmento de reta que liga $(0,0)$ a $(0,-\sqrt{3})$ deve ser identificado com o segmento de reta que liga $(0,0)$ a $(0, \sqrt{3})$ trocando A por B pois neste caso 
$\overline{\mathrm{AC}}=\overline{\mathrm{BC}}$.

Vamos mostrar que o espaço quociente é homeomorfo a $S^{2}$ (obtida por identificação da meia lua) à qual fica colado o ponto $\omega$, imagem da classe de $\{(A, A, A), A \in C\}$

Desde que a relação de afinidade $(\sim)$ identifica pontos, precisamos colocar uma estrutura topológica no espaço resultante. Seja $\rho: \mathbf{c}^{3} \rightarrow \mathbb{c}^{3} / \sim=$ $S^{2} v\{\omega\}$ a identificação dos coeficientes dos polinômios com os elementos do quociente pela relação de afinidade.

Afirmamos que:

1. 0 ünico aberto que contém $\omega$ é o espaço todo $s^{2} v\{\omega\}$.

2. Os outros abertos são os abertos em $\mathrm{s}^{2}$.

De fato: 1. Suponhamos que A seja uma raiz tripla, então o polinômio é do ti po: $(x-A)^{3}=x^{3}-3 A x^{2}+3 A^{2} x-A^{3}$.

Logo $\rho^{-1}(\omega)=\left\{(a, b, c) \in \mathbb{C}^{3} \mid a=-3 A, b=3 A^{2}, C=-A^{3}\right.$, com $\left.A \in c^{1}\right\}$

Queremos éncontrar $\Omega$ aberto em $S^{2} u\{\omega\}$ talque $\omega \in \Omega$.

Lembremos que um conjunto $\Omega \dot{e}$ um aberto no quociente $s^{2} \cup\{\omega\}$ se $\rho^{-1}(\omega)$ é um aberto em $\mathbb{C}^{3}$ (em $\mathbb{C}^{3}$ utilizaremos a topologia usual).

Temos que $(0,0,0) \in \rho^{-1}(\omega)$. Dado um polinômio qualquer com coefi cientes em $\mathfrak{C}$, que não tenha raiz tripla, obtemos com suas raízes um triângu1o $\mathrm{ABC}$ que pela relação de afinidade é levado em $(-1,1, \mathrm{C})$. Porém para qual quer $\varepsilon>0$, o polinömio $X^{3}-\varepsilon C X^{2}-\varepsilon^{2} X+\varepsilon^{3} C=(X+\varepsilon)(X-\varepsilon)(X-\varepsilon C)$ tem raizes $(\varepsilon,-\varepsilon,-\varepsilon C)$ que é levado no mesmo triângulo $(-1,1, C)$ pela relação de afinidade.

Observamos que para qualquer aberto em torno de $(0,0,0)$, existe $\varepsilon>0$ tal que $\left(-\varepsilon C,-\varepsilon^{2}, \varepsilon^{3}\right)$ está nele contido, assim $\left(-\varepsilon C,-\varepsilon^{2}, \varepsilon^{3}\right) \in \mathbf{C}^{3}$ estä tão próximo de $(0,0,0)$ quanto se queira. Como o polinómio foi tomado arbitraria- 
mente temos que todos os polinômios são equivalentes a representantes num aberto qualquer em torno de $(0,0,0)$, isto é, para que $\Omega$ seja aberto em $s^{2} u\{\omega\}$ é necessário que $\rho^{-1}(\Omega)$ contenha representantes de todas as classes de polinômios de $c^{3}$, logo $\Omega=s^{2} v\{w\}$.

Também podiamos dizer que em qualquer vizinhança de $\omega$ podemos encontrar triāngulos semelhantes a qualquer triāngulo dado.

Assim 1. fica demonstrado.

2. Queremos inicialmente os abertos no interior de $\Sigma$, vamos mastrar que um aberto neste caso em torno de $C \in$ int $\Sigma$ é dado por:

$$
\left.U=! C^{\prime} \in \operatorname{int} \Sigma: \mid C-C \eta<\varepsilon\right\} \text {. }
$$

Com efeito: Dado um polinómio $P: X^{3}+\alpha X^{2}+\beta X+\gamma$ podemos encontrar coeficientes $\alpha^{\prime}, \beta^{\prime}, \gamma^{\prime}$ tais que $\left|\alpha-\alpha^{\prime}\right|<\delta$; $\left|\beta-\beta^{\prime}\right|<\delta$ e $\left|\gamma-\gamma^{\prime}\right|<\delta$ onde $\delta$ é escolhido de maneira que as raízes $\left(A^{\prime}, B^{\prime}, C^{\prime}\right)$ do novo polinómio $P^{\prime}$ estejam muito próximas das raízes originais $(A, B, C)$ e com isto fazer com que após as duas identificações $A$ com $\omega, B$ com $\sigma$ e $C \operatorname{com} \tilde{C}$ em seguida $A^{\prime} \operatorname{com} \omega, \bar{B}^{\prime} \operatorname{com} \sigma$ e $C$ com $\chi^{\prime}$ conseguimos obter $\left\{\tilde{C}^{\prime} \in U:\left|\tilde{C}-\tilde{C}^{\prime}\right|\left\langle\varepsilon^{\prime}\right\}=\tilde{D}\right.$. Logo se

$$
W=\left\{\left(\alpha^{\prime}, \beta^{\prime}, \gamma^{\prime}\right) \in C^{3}:\left|\alpha-\alpha^{\prime}\right|<\delta,\left|\beta-\beta^{\prime}\right|<\delta,\left|\gamma-\gamma^{\prime}\right|<\delta\right\} \text { en- }
$$

tão $\rho(W) \subset \tilde{D}$, e assim $W \subset \rho^{-1}(\tilde{D}) \subset \rho^{-1}(U)$ e portanto $\rho^{-1}(U)$ é um aberto.

Seja agora um ponto $c=\left(c_{1}, c_{2}\right) \in$ Fr $\Sigma$ tal que $\sqrt{\left(c_{1}+1\right)^{2}+c_{2}{ }^{2}}=2$. Queremos mostrar que os abertos de $\Sigma$ que contém $C$ são da forma

$$
\mathrm{V}=\left\{\mathrm{C}^{\prime} \epsilon \Sigma:\left|\mathrm{C}-\mathrm{C}^{\prime}\right|<\varepsilon \text { ou }\left|\overline{\mathrm{C}}-\mathrm{C}^{\prime}\right|<\varepsilon\right\} \text {, onde } \overline{\mathrm{C}} \dot{\mathrm{e}} \text { o conjugado }
$$
de $C$.

Consideremos o mesmo aberto $W$ em $C^{3}$ tal que $\delta$ é escolhido de maneira que $\rho\left(\alpha^{\prime}, B^{\prime}, \gamma^{\prime}\right) \stackrel{(*)}{C}\left\{C^{\prime} \in \Sigma:\left|C-C^{\prime}\right|<\varepsilon\right.$ ou $\left.\left|\bar{C}-C^{\prime}\right|<\varepsilon\right\}$. Iogo $\rho(W) \subset V$ então $W \subset \rho^{-1}(V)$ e assim $V$ é aberto em $\Sigma$. 
(*) é possivel pois se as raîzes $A^{\prime}, B^{\prime}, C^{\prime}$ do polinômio $x^{3}+\alpha^{\prime} X^{2}+B^{\prime} X+\gamma^{\prime}$ forem tais que $\overline{A^{\top} C^{\top}}>\overline{A^{\top} B^{\top}}$ trocamos $C^{\prime}$ por $B^{\prime}$ e assim o novo $C^{\prime} e^{e}$ levado próximo de $\overline{\mathrm{C}}$ em $\Sigma$.

Tomemos agora um ponto $\mathrm{C}=\left(c_{1}, c_{2}\right) \in$ Fr $\Sigma$ tal que $c_{1}=0$. Queremos mostrar que os abertos de $\Sigma$ que contēm $C$ são da forma de $V$ definido acima.Pa ra isto resta justificar (*).

(*) aqui 'è possível pois se as raízes $A^{\prime}, B^{\prime}, C^{\prime}$ do polinômio $X^{3}+\alpha^{\prime} X^{2}+B^{\prime} X+$ $+\gamma^{\prime}$ forem tais que $\overline{A^{\top} C^{\top}}<\overline{B^{\top} C^{\top}}$ trocamos $A^{\prime}$ por $B^{\prime}$ e assim o novo $C^{\prime} e^{e}$ levado pröximo de $c_{o}$ em $\Sigma$.

\$2. O espaço de órbitas da parte principal $\mathrm{f}_{\mathrm{O}}$

Aqui estudaremos em dois exemplos a topologia de subconjuntos $\Theta \subset \Omega$, obtendo a identificação com espaços topológicos simples.

\section{$2.1-$ Exemplo 1}

Mostramos no Capitulo I (Teorema 1.10) que se $j_{4} f=x^{4}+a x^{2} y^{2}+y^{4}$, $\mathrm{a}^{2} \neq 4$, então $\mathrm{E} \in \mathrm{X}_{9}$ isto é, $\mathrm{f}=\mathrm{x}^{4}+a \mathrm{x}^{2} \mathrm{y}^{2}+\mathrm{y}^{4}$.

Vamos agora encontrar os possiveis elementos de uma mesma örbita após a ação de difeomorfismos que deixam fixo $f_{0}$ a menos que sejam germes de uma mesma órbita. Em seguida passando ao quociente pela relação, "germes da mesma órbita", encontraremos a geometria do espaço das örbitas.

$$
\text { Façamos a mudança: }\left\{\begin{array}{l}
x=\alpha x^{\prime}+\beta y^{\prime} \\
y=\gamma x^{\prime}+\delta y^{\prime}
\end{array}\right.
$$

Logo:

$$
\begin{aligned}
& x^{4}+a x^{2} y^{2}+y^{4}=\left(\alpha x^{\prime}+\beta y^{\prime}\right)^{4}+a\left(\alpha x^{\prime}+\beta y^{\prime}\right)^{2}\left(\gamma x^{\prime}+\delta y^{\prime}\right)^{2}+\left(\gamma x^{\prime}+\delta y^{\prime}\right)^{4}= \\
& =\left(\alpha^{4}+a \alpha^{2} \gamma^{2}+\gamma^{4}\right) x^{\prime 4}+\left(4 \alpha^{3} \beta+2 a \alpha^{2} \gamma \delta+2 a \alpha \beta \gamma^{2}+4 \gamma^{3} \delta\right) x^{\prime} y^{\prime}+
\end{aligned}
$$


$+\left(6 \alpha^{2} \beta^{2}+a \alpha^{2} \delta^{2}+4 a \alpha \beta \gamma \delta+a \beta^{2} \gamma^{2}+6 \gamma^{2} \delta^{2}\right) x^{\prime 2} y^{\prime 2}+\left(4 \alpha \beta^{3}+2 a \alpha \beta \delta^{2}+2 a \beta \gamma^{2} \delta+4 \gamma \delta^{3}\right) x^{\prime} y \prime^{3}+$ $+\left(\beta^{4}+a \beta^{2} \delta^{2}+\delta^{4}\right) y^{\prime 4}$. Queremos que :

$\left\{\begin{array}{l}\alpha^{4}+a \alpha^{2} \gamma^{2}+\gamma^{4}=1 \\ 4 \alpha^{3} \beta+2 a \alpha^{2} \gamma \delta+2 a \alpha \beta \gamma^{2}+4 \gamma^{3} \delta=0 \\ 6 \alpha^{2} \beta^{2}+a \alpha^{2} \delta^{2}+4 a \alpha \beta \gamma \delta+a \beta^{2} \gamma^{2}+6 \gamma^{2} \delta^{2}=b \\ 4 \alpha \beta^{3}+2 a \alpha \beta \delta^{2}+2 a \beta^{2} \gamma \delta+4 \gamma \delta^{3}=0 \\ \beta^{4}+a \beta^{2} \delta^{2}+\delta^{4}=1\end{array}\right.$

Sejam $A=\alpha / \gamma$ e $B=B / \delta$, assimficamos com:

$$
\left\{\begin{array}{l}
A^{4}+a A^{2}+1=1 / \gamma^{4} \\
4 A^{3} B+2 a A^{2}+2 a A B+4=0 \\
6 A^{2} B^{2}+a A^{2}+4 a A B+2 a B^{2}+6=b /\left(\gamma^{2} \delta^{2}\right) \\
4 A B^{3}+2 a A B+a B^{2}+4=0 \\
B^{4}+a B^{2}+1=1 / \delta^{4}
\end{array}\right.
$$

Subtraindo $\left(2^{\prime}\right)$ de $\left(4^{\prime}\right)$ obtemos:

$$
\left(A^{2}-B^{2}\right)(2 A B+a)=0 \text {. }
$$

$A=B$ não pode ocorrer, pois neste caso $\alpha \delta=\beta \gamma$ e não teríamos uma boa mudança de coordenadas.

Suponhamos: (a) $A=-B$ e (b) $A=-a / 2 B$.

(a) Tomemos $\mathrm{A}=-\mathrm{B}$ em (2')

$$
\begin{aligned}
& -4 A^{4}-2 a A^{2}+2 a A^{2}+4=0 \Rightarrow A^{4}=1 \Rightarrow \gamma=\varepsilon \alpha, \text { onde } \\
& \varepsilon=\{1, i,-1,-i\} .
\end{aligned}
$$

Substituindo $\gamma=\varepsilon \alpha$ em (1) obtemos $\alpha^{4}+a \alpha^{2}(\varepsilon \alpha)^{2}+(\varepsilon \alpha)^{4}=1$

Ficamos então com as equações:

$$
\begin{cases}\alpha^{4}+a \alpha^{4}+\alpha^{4}=1 & \text { (i) } \varepsilon= \pm 1 \text { ou } \\ \alpha^{4}-a \alpha^{4}+\alpha^{4}=1 & \text { (ii) } \varepsilon= \pm i\end{cases}
$$


Temos ainda que $(\alpha / \gamma)=(\beta / \delta) \Rightarrow(\alpha / \varepsilon \alpha)=(-\beta / \delta) \Rightarrow \delta=-\beta$, em (5), obtemos: $\beta^{4}+a \beta^{2}(-\varepsilon \beta)^{2}+(-\varepsilon \beta)^{4}=1 \Rightarrow$

$$
\Rightarrow \begin{cases}\beta^{4}+a \beta^{4}+\beta^{4}=1 & \text { (iii) se ocorre (i) } \\ \dot{\beta}^{4}-a \beta^{4}+\beta^{4}=1 & \text { (iv) se ocorre (ii) }\end{cases}
$$

19 Caso: De (i) $\alpha^{4}=1 /(2+a) \Rightarrow \alpha=\nu(1 /(2+a))^{1 / 4}$, onde $\nu=\{1, i,-1,-i\}$. Logo $\gamma=\varepsilon \alpha=\varepsilon \nu(1 /(2+a))^{1 / 4} \quad(v) ; \varepsilon= \pm 1$

De $(\mathrm{iii}) \beta^{4}=1 /(2+a) \Rightarrow \beta=\mu\left(1 /(2+a)^{1 / 4}\right.$, onde $\mu=\{1, i,-1,-i\}$

Logo: $\delta=-\varepsilon \beta=-\varepsilon \mu(1 /(2+a))^{1 / 4}(v i)$

(v) e (vi) em (3):

$6 \nu^{2} \mu^{2}(1 /(2+a))+a \nu^{2}(-\varepsilon \mu)^{2}(1 /(2+a))+4 a \nu \mu(\varepsilon \nu)(-\varepsilon \mu)(1 /(2+a))+$

$+a \mu^{2}(\varepsilon \nu)^{2}(1 /(2+a))+6(\varepsilon \nu)^{2}(-\varepsilon \mu)^{2}(1 /(2+a))=b \Rightarrow$ $\varepsilon=+1$

$\Rightarrow\left\{\begin{array}{l}6\left(\frac{1}{2+a}\right)+a\left(\frac{1}{2+a}\right)+a\left(\frac{1}{2+a}\right)-4 a\left(\frac{1}{2+a}\right)+6\left(\frac{1}{2+a}\right)=b \\ 6\left(\frac{1}{2+a}\right)+a\left(\frac{1}{2+a}\right)+a\left(\frac{1}{2+a}\right)-4 a\left(\frac{1}{2+a}\right)+6\left(\frac{1}{2+a}\right)=-b\end{array} \Rightarrow\right.$

$\Rightarrow \quad b=\frac{12-2 a}{2+a} \quad$ ou $\quad b=\frac{-12+2 a}{2+a}$

20 Caso: De (ii) $\alpha^{4}=1 /(2-a) \Rightarrow \alpha=\nu(1 /(2-a))^{1 / 4} \cdot$ Logo:

$$
\begin{aligned}
& \gamma=\varepsilon \alpha=\varepsilon \nu(1 /(2-a))^{1 / 4} \quad(v i i) ; \varepsilon= \pm i \\
& \text { De }(i i i) \beta^{4}=1 /(2-a) \Rightarrow \beta=\mu\left(1 /(2-a)^{1 / 4}\right. \text {. Logo: } \\
& \delta=-\varepsilon \beta=-\varepsilon \mu(1 /(2-a))^{1 / 4} \quad \text { (viii) }
\end{aligned}
$$

(vii) e (viii) em (3)

$$
\Rightarrow\left\{\begin{array}{l}
6\left(\frac{1}{2-a}\right)-a\left(\frac{1}{2-a}\right)+4 a\left(\frac{1}{2-a}\right)-a\left(\frac{1}{2-a}\right)+6\left(\frac{1}{2-a}\right)=b \\
6\left(\frac{1}{2-a}\right)-a\left(\frac{1}{2-a}\right)+4 a\left(\frac{1}{2-a}\right)-a\left(\frac{1}{2-a}\right)+6\left(\frac{1}{2-a}\right)=-b
\end{array} \Rightarrow\right.
$$




$$
\Rightarrow b=\frac{12+2 a}{2-a} \quad \text { ou } \quad b=\frac{-12-2 a}{2-a}
$$

30. Caso: Pode ocorrer $\alpha=0$. Neste caso, substituindo em (1) obtemos $\gamma=\varepsilon$ em (2) obtemos $\delta=0$ que em (5) nos dä $\beta=\mu$, em (3) b= *Observação: obtemos reșultados anälogos para $\beta=0$.

Esgotamos assim todas as possibilidades de (a).

(b) Tomemos $\mathrm{A}=-\mathrm{a} / 2 \mathrm{~B}$ em (2'), obtemos:

$$
\begin{aligned}
& 4(-a / 2 B)^{3} B+2 a(-a / 2 B) B+2 a(-a / 2 B)^{2}+4=0 \Rightarrow \\
& \Rightarrow-\left(a^{3} / 2 B^{2}\right)-a^{2}-\left(a^{3} / 2 B\right)+4=0 \Rightarrow a= \pm 2 \text {, que nos dá: } \\
& x^{4} \pm 2 x^{2} y^{2}+y^{4} \text {, que possui pontos singulares degenerados. }
\end{aligned}
$$

Concluímos então que os germes $x^{4}+b_{i} x^{2} y^{2}+y^{4}, i=1,2.3,4,5$ e 6 , onde $b_{1}=a ; b_{2}=-a ; b_{3}=\frac{12-2 a}{2+a} ; b_{4} \doteq \frac{-12+2 a}{2+a} ; b_{5} \doteq \frac{12+2 a ;}{2-a} b_{6}=\frac{-12-2 a}{2-a} \quad$ são equivalentes.

Representando $x^{4}+a x^{2} y^{2}+y^{4}$ pelo ponto a do plano complexo obte mos na figura A uma região (hachuriada) que representa todas as classes de equivaléncia dos germes, isto è, a região contém representantes de todas as diferentes örbitas (sistema de representantes).

Demonstraremos agora a veracidade deste fato:

Suponhamos que $a=p+q i$, ficamos então com

$b_{1}=(p, q) ; b_{2}=(-p,-q) ; b_{3}=\left(\frac{24+8 p-2 p^{2}-2 q^{2}}{(2+p)^{2}+q^{2}}, \frac{-16 q}{(2+p)^{2}+q^{2}}\right) ;$

$b_{4}=\left(\frac{-24-8 p+2 p^{2}+2 q^{2}}{(2+p)^{2}+q^{2}}, \frac{16 q}{(2+p)^{2}+q^{2}}\right) ; b_{5}=\left(\frac{24-8 p-2 p^{2}-2 q^{2}}{(2-p)^{2}+q^{2}}, \frac{16 q}{(2-p)^{2}+q^{2}}\right) ;$

$b_{5}=\left(\frac{-24+8 p+2 p^{2}+2 q^{2}}{(2-p)^{2}+q^{2}}, \frac{-16 q}{(2-p)^{2}+q^{2}}\right)$. 


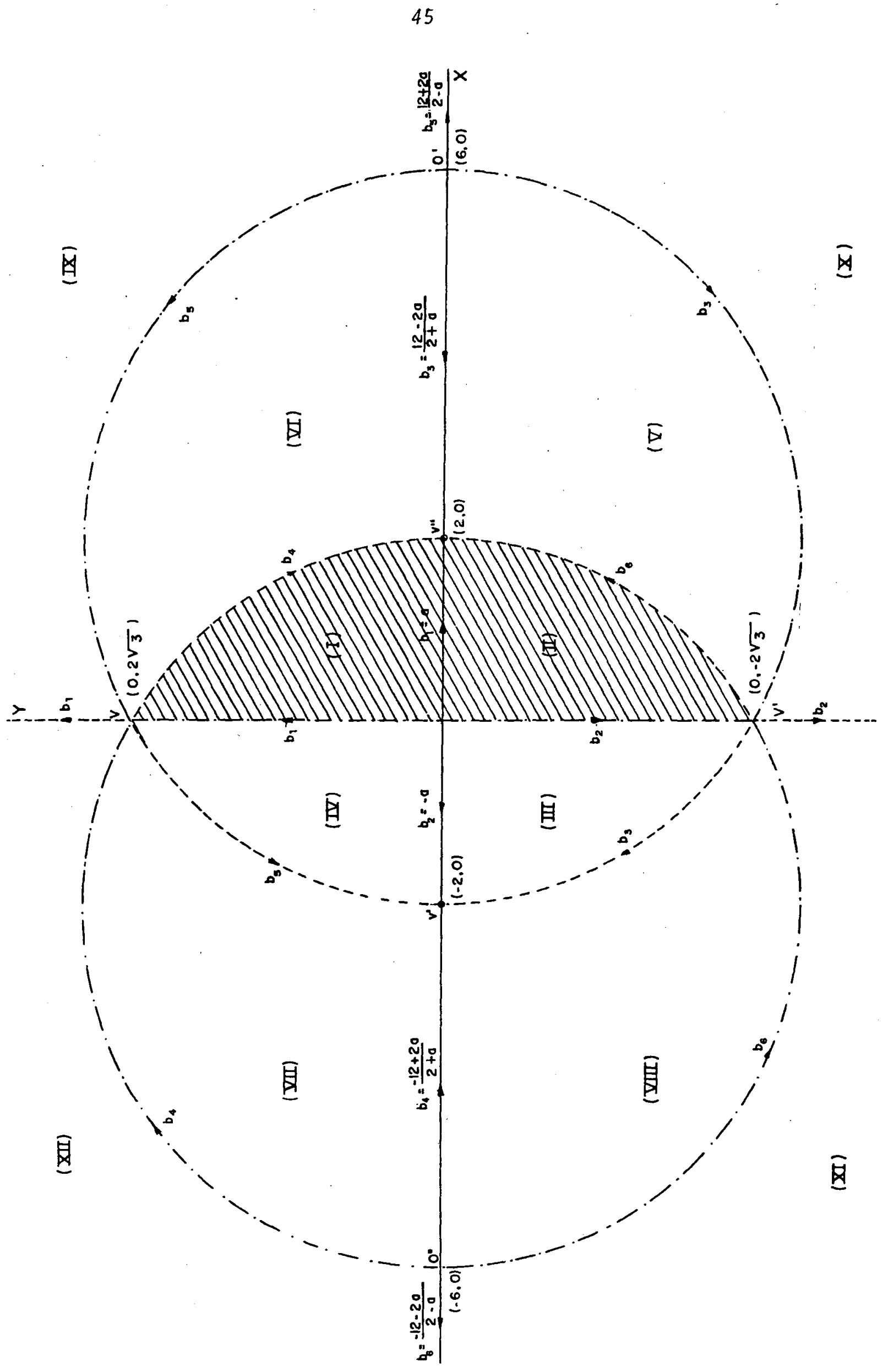

FIGURA A 
Consideremos as regiões em $\mathbb{C}^{1}$ dadas por:
$\left\{\begin{array}{l}x \geqq 0 \\ y \geqq 0 \\ (x+2)^{2}+y^{2} \leqq 16\end{array}\right.$
(II) $\left\{\begin{array}{l}x \geqq 0 \\ y \leqq 0 \\ (x+2)^{2}+y^{2} \leqq 16\end{array}\right.$

Vamos tomar um ponto $(p, q)$ tal que $b_{1}=(p, q) \epsilon(I)$ e vamos analisar em quais regiões aparecem $b_{2}, b_{3}, b_{4}, b_{5}$ e $b_{6}$.

$$
\begin{aligned}
& \mathrm{b}_{2}=\left(\mathrm{p}_{2}, \mathrm{q}_{2}\right)=(-\mathrm{p},-\mathrm{q}) \Rightarrow\left\{\begin{array}{l}
\mathrm{p}_{2} \leqq 0 \\
q_{2} \leqq 0 \\
\left(p_{2}-2\right)^{2}+q_{2}^{2} \leqq 16
\end{array} \quad\right. \text { região (III) } \\
& \mathrm{b}_{3}=\left(\mathrm{p}_{3}, \mathrm{q}_{3}\right) \Rightarrow\left\{\begin{array}{l}
\mathrm{p}_{3}^{i}=\frac{24+8 p-2 \mathrm{p}^{2}-2 \mathrm{q}^{2}}{(2+\mathrm{p})^{2}+\mathrm{q}^{2}} \\
\mathrm{q}_{3}=\frac{-16 \mathrm{q}}{(2+\mathrm{p})^{2}+\mathrm{q}^{2}}
\end{array}\right.
\end{aligned}
$$

Claramente $q_{3} \leqq 0$. Mostraremos que: $\left\{\begin{array}{l}\left(\mathrm{p}_{3}+2\right)^{2}+\mathrm{q}_{3}{ }^{2} \geqq 16 \\ \left(\mathrm{p}_{3}-2\right)^{2}+\mathrm{q}_{3}{ }^{2} \leqq 16\end{array}\right.$.

isto $\bar{e}, b_{3}$ pertence a região (V)

De fato: 1. $\left[\frac{24+8 p-2 p^{2}-2 q^{2}}{(2+p)^{2}+q^{2}}+2\right]^{2}+\left[\frac{-16 q}{(2+p)^{2}+q^{2}}\right]^{2}=\frac{16}{(2+p)^{2}+q^{2}} \geqq 16$, pois

por hipötese $(2+p)^{2}+q^{2} \leqq 16$. Portanto 1. está verificado.

2. $\left[\frac{24+8 p-2 p^{2}-2 q^{2}}{(p+2)^{2}+q^{2}}-2\right]^{2}+\left[\frac{-16 q}{(2+p)^{2}+q^{2}}\right]=\underbrace{16 \underbrace{\left[\left(4-p^{2}-q^{2}\right)^{2}+16 q^{2}\right]}_{A}}_{M}$

Mostremos que $\mathrm{A} \leqq 1$

$$
\left(4-p^{2}-q^{2}\right)^{2}+16 q^{2}=\left(4-\left(p^{2}+q^{2}\right)\right)^{2}+16 q^{2}=16-8 p^{2}+8 q^{2}+\left(p^{2}+q^{2}\right)^{2}
$$




$$
\begin{aligned}
& {\left[(p+2)^{2}+q^{2}\right]^{2}=\left(p^{2}+4 p+4+q^{2}\right)^{2}=\left(p^{2}+q^{2}+4(p+1)\right)^{2}=} \\
& =\left(p^{2}+q^{2}\right)^{2}+8\left(p^{2}+q^{2}\right) \underbrace{(p+1)}_{\geqq 1}+16 \underbrace{(p+1)^{2}}_{\geqq 1} \geqq \\
& \geqq\left(p^{2}+q^{2}\right)^{2}+8\left(p^{2}+q^{2}\right)+16 \geqq\left(p^{2}+q^{2}\right)-8 p^{2}+8 q^{2}+16= \\
& (*)\left(4-p^{2}-q^{2}\right)^{2}+16 q^{2} .
\end{aligned}
$$

Portanto $A \leqq 1$ e assim 2. fica demonstrado.

$$
b_{4}=\left(p_{4}, q_{4}\right) \Rightarrow\left\{\begin{array}{l}
p_{4}=\frac{-24-8 p+2 p^{2}+2 q^{2}}{(2+p)^{2}+q^{2}} \\
q_{4}=\frac{16 q}{(2+p)^{2}+q^{2}}
\end{array}\right.
$$

Claramente $q_{4} \geqq 0$. Mostraremos que: $\left\{\begin{array}{l}\left(\mathrm{p}_{4}-2\right)^{2}+\mathrm{q}_{4}{ }^{2} \geqq 16 \\ \left(\mathrm{p}_{4}+2\right)^{2}+\mathrm{q}_{4}{ }^{2} \leqq 16\end{array}\right.$. isto $\dot{e}, b_{4}$ pertence a região (VII).

$$
\begin{aligned}
& \text { Observando que: }\left(p_{4}-2\right)^{2}=\left(p_{3}+2\right)^{2} \quad 3 \text { fica demonstrado. } \\
&\left(p_{4}+2\right)^{2}=\left(p_{3}-2\right)^{2} \quad 4 \text {. fica demonstrado. } \\
& b_{5}=\left(p_{5}, q_{5}\right) \Rightarrow\left\{\begin{array}{l}
p_{5}=\frac{24-8 p-2 p^{2}-2 q^{2}}{(2-p)^{2}+q^{2}} \\
q_{5}=\frac{16 q}{(2-p)^{2}+q^{2}}
\end{array}\right.
\end{aligned}
$$

Claramente $q_{5} \geqq 0$. Mostremos que: $\begin{cases}p_{5} \geqq 0 & 5 \text {. } \\ \left(p_{5}-2\right)^{2}+q_{5}{ }^{2} \geqq 16 & 6 .\end{cases}$

isto $\bar{e}, b_{5}$ pertence a região (IX).

De Fato: para 5 . basta mostrar que $24-8 p-2 p^{2}-2 q^{2} \geqq 0$ ou seja $12-4 p-p^{2}-q^{2} \geqq$ $\geqq 0$.

$12-4 p-p^{2}-q^{2}=12+4-4-4 p-p^{2}-q^{2}=16-\underbrace{\left[(p+2)^{2}+q^{2}\right]}_{\leqq 16} \geqq 0$

Portanto 5. fica demonstrado. 


$$
\begin{aligned}
& \text { para } 6\left[\frac{24-8 p-2 p^{2}-2 q^{2}}{(2-p)^{2}+q^{2}}-2\right]^{2}+\left[\frac{16 q}{(2-p)^{2}+q^{2}}\right]= \\
& =16 \frac{\left[\left(4-p^{2}-q^{2}\right)^{2}+16 q^{2}\right]}{\left[(2-p)^{2}+q^{2}\right]}=B
\end{aligned}
$$

Mostremos que $B \geqq 1$.

$\operatorname{De}(*)\left(4-p^{2}-q^{2}\right)^{2}+16 q^{2}=16-8 p^{2}+8 q^{2}+\left(p^{2}+q^{2}\right)^{2}$

Por outro lado

$$
\begin{aligned}
{\left[(p-2)^{2}+q^{2}\right]^{2} } & =\left(p^{2}+q^{2}\right)^{2}+8\left(p^{2}+q^{2}\right)(1-p)+16(1-p)^{2} \leqq \\
& \leqq 16-8 p^{2}+8 q^{2}+\left(p^{2}+q^{2}\right)^{2(*)}=\left(4-p^{2}-q^{2}\right)^{2}+16 q^{2}
\end{aligned}
$$

Logo $B \geqq 1$ e 6 . fica demonstrado se demonstrarmos $(* *)$.

(**) fica demonstrado se demonstrarmos que:

$$
\left(16-8 p^{2}+8 q^{2}\right)-\left[8\left(p^{2}+q^{2}\right)(1-p)+16(1-p)^{2}\right] \geqq 0 \text { e isto é verdadeiro. }
$$

Com efeito: $16-8 p^{2}+8 q^{2}-\left[8 p^{2}+8 q^{2}-8 p\left(p^{2}+q^{2}\right)+16\left(1-2 p+p^{2}\right)\right]=$

$$
\begin{aligned}
& =8 p\left(p^{2}+q^{2}\right)+32 p \geqq 0 \\
& b_{6}=\left(p_{6}^{3}, q_{6}\right) \Rightarrow\left\{\begin{array}{l}
p_{6}=\frac{-24+8 p+2 p^{2}+2 q^{2}}{(p-2)^{2}+q^{2}} \\
q_{6}=\frac{-16 q}{(p-2)^{2}+q^{2}}
\end{array}\right.
\end{aligned}
$$

claramente $q_{6} \leqq 0$. Mostremos que: $\begin{cases}p_{6} \leqq 0 & 7 . \\ \left(p_{6}+2\right)^{2}+q_{6} \geqq 16 & 8 .\end{cases}$

is to $\hat{\mathrm{e}}, \mathrm{b}_{6}$ pertence a regiāo $(\mathrm{XI})$.

Como $\mathrm{p}_{6}=-\mathrm{p}_{5}$ de 5. segue 7..

Observando que $\left(p_{6}+2\right)^{2}=\left(p_{5}-2\right)^{2}$ de 6 . obtemos 8 .

Concluímos então que, dado um ponto em (I) temos mais 5 (cinco)pon tos distribuídos nas regiōes (III), (V), (VII), (IX) e (XI), pertencentes a mesma örbita. 
Fazendo agora um estudo semelhante para um ponto $b_{1}=(p, q)$ perten cente a região (II), temos que:

$$
\begin{aligned}
& \mathrm{b}_{2}=\left(\mathrm{p}_{2}, \mathrm{q}_{2}\right) \text { pertence a região (IV) } \\
& \mathrm{b}_{3}=\left(\mathrm{p}_{3}, \mathrm{q}_{3}\right) \text { pertence a região (VI) } \\
& \mathrm{b}_{4}=\left(\mathrm{p}_{4}, \mathrm{q}_{4}\right) \text { pertence a região (VIII) } \\
& \mathrm{b}_{5}=\left(\mathrm{p}_{5}, \mathrm{q}_{5}\right) \text { pertence a região (X) } \\
& \mathrm{b}_{6}=\left(\mathrm{p}_{6}, \mathrm{q}_{6}\right) \text { pertence a região (XII) }
\end{aligned}
$$

As demonstrações são semelhantes as dos casos anteriores.

Portanto temos que se um ponto estâ em (II), então existem outros 5 (cinco) pontos distribuidos nas regiões (IV), (VI), (VIII), (X), (XII) per tencentes a mesma órbita.

Como as transformações:

$\mathrm{b}_{1}=\mathrm{a} ; \mathrm{b}_{2}=-\mathrm{a} ; \mathrm{b}_{3}=\frac{12-2 \mathrm{a}}{2+\mathrm{a}} ; \mathrm{b}_{4}=\frac{-12+2 \mathrm{a}}{2+\mathrm{a}} ; \mathrm{b}_{5}=\frac{12+2 \mathrm{a}}{2-\mathrm{a}} ; \mathrm{b}_{6}=\frac{-12-2 \mathrm{a}}{2-\mathrm{a}}$, onde $\mathrm{b}_{1}$, $b_{2}, b_{3}, b_{4}, b_{5}, b_{6} \in \mathbb{C}$ são de MUbius, as correspondências entre os pontos de (I) e (II) e os correspondentes contra-domínios encontrados acima são biunívocas.

Com isso verificamos o afirmado, isto é, que as regiões (I) e (II) contém representantes de todas as örbitas do germe $x^{4}+a x^{2} y^{2}+y^{4}$.

Observamos que se $b_{1}$ caminha pelo segmento de reta de $(0.0)$ a $(0,2 \sqrt{3})$ então $b_{2}$ caminha de $(0,0)$ a $(0,-2 \sqrt{3})$, logo devemos identificar estes dois segmentos; além disso quando $b_{1}$ caminha de $(0,2 \sqrt{3})$ para $(0, \infty)$ temos que $b_{4}$ caminha de $(0,2 \sqrt{3})$ para $(2,0)$ pelo arco $(x+2)^{2}+y^{2}=16$ e $b_{5}$ caminha de $(0,-2 \sqrt{3})$ para $(2,0)$ pelo arco $(x+2)^{2}+y^{2}=16$ que portanto também podem ser identificados.

Vamos mostrar que o quociente é homeomorfo a $\mathrm{s}^{2}$. 
Os pontos que poderiam gerar düvidas são os pontos de fronteira. Pa ra esta demonstração, denotaremos a identificação de $\mathbf{C}$ com o espaço quociente por $\phi: \mathbf{C} \rightarrow \mathbf{c} / \imath=s^{2}$.

1) Consideremos uma bola aberta $B_{1}^{1}$ com centro em $(0, y)$, onde $0<y<2 \sqrt{3}$. e contida nas regiões (I) e (IV). No quociente, a parte da bola con tida em (IV) é levada na parte da bola $\mathrm{B}_{2}^{1}$ com centro em $(0,-y)$ contida em (II). Afirmamos que o conjunto $\mathrm{A}_{1}$ formado pelas partes das bolas contidas em (I) e (II) é um aberto no quociente. De fato:

$\phi^{-1}\left(A_{1}\right)=B_{1}^{1} \cup B_{2}^{1} \cup B_{3}^{1} \cup B_{4}^{1} \cup B_{5}^{1} \cup B_{6}^{1}$ por continuidade, onde por exemplo $B_{3}^{1}$ é a bola com centro em $\left(\frac{24-2 y^{2}}{2+y^{2}},-\frac{16 y}{2+y^{2}}\right)$, a! parte (I) é levada em (V) e a de (II) em (X).

2) Consideremos agora uma bola aberte $\mathrm{B}_{4}^{2}$ com centro em $\left(\mathrm{x}_{0}, \mathrm{y}_{0}\right)$ onde $\left(x_{0}+2\right)^{2}+y_{0}^{2}=16, x_{0}>0$ e $y_{0}>0$. No quociente a parte da bola contida em (VI) é levada na parte da bola $B_{6}^{2}$ com centro em $\left(x_{0},-y_{0}\right)$ contida em (II). Afirmamos que o conjunto $A_{2}$ formado pelas partes de $B_{4}^{2}$ e $B_{6}^{2}$ contidas em (I) e (II) respectivamente é um aberto no quociente. De fato: $\phi^{-1}\left(A_{2}\right)=B_{1}^{2} \cup B_{2}^{2} \cup B_{3}^{2} \cup B_{4}^{2} \cup B_{5}^{2} \cup B_{6}^{2}$ por continuidade, onde por exemplo $B_{1}^{2}$ é a bola com centro em $\left(0, \sqrt{\frac{4 x_{0}+24}{2-x_{o}}}\right)$ tal que a parte de $A_{2}$ em (I) é levada em $(X I)$ e a de (II) em (XII).

3) Uma bola $\mathrm{B}_{1}^{3}$ com centro em $(2,0)$ contida em (I) e (II) coincide com ela mesma no quociente. Afirmamos que $B_{1}^{3}$ é aberto no quociente. De fato:

$\phi^{-1}\left(B_{1}^{3}\right)=B_{1}^{3} \cup B_{2}^{3} \cup B_{3}^{3} \cup B_{4}^{3} \cup B_{5}^{3} \cup B_{6}^{3}$ por continuidade, onde por exemplo $B_{6}^{3}$ tem centro em $\left(\frac{-24+8 x+2 x^{2}}{(2-x)^{2}}, 0\right)$, onde a parte de (I) é levada em (XI) e a 
parte de (II) em (XII).

4) Tomemos uma bola aberta $B_{0}^{4}$ com centro em $(0,0)$ contida em (I), (II), (III) e (IV). No quociente a parte de $B_{0}^{4}$ em (III) è levada na parte da bola $B_{0}^{4}$.em (I) e a de (IV) em (II). Afirmamos que o semi-círculo $A_{4}$ de (I) $\ddot{e}$ um aberto no quociente. De fato:

$\phi^{-1}\left(A_{4}\right)=B_{0}^{4} \cup B_{0}^{4}, \cup B_{0}^{4}$, por continuidade, onde $B_{0}^{4}$, tem centro em $(6,0), a$ parte de (I) è levada em (V) e (IX) e a parte de (II) em (VI) e (X), jà B B $^{4}$, tem centro em $(-6,0)$ a parte de (I) é levada em (VII) e (XI) e a parte de (II) em (VIII) e (XII).

5) Uma bola $B_{2}^{5}$ com centro em $(2,0)$ contida em (I), (II), (V) e (VI), no quociente a parte de (V) vai em (I) e a parte de (VI) na parte de $\mathrm{B}_{2}^{5}$ em (II). Afirmamos que o conjunto $\mathrm{A}_{5}$ constituido da parte de $\mathrm{B}_{2}^{5}$ em (I) e (II) è um aberto no quociente. De fato:

$\phi^{-1}\left(A_{5}\right)=B_{2}^{5} \cup B_{-2}^{5} \cup B_{\omega}^{5}$ por continuidade, onde $B_{-2}^{5}$ e a bola com centro em $(-2,0)$ e contida em (III), (IV), (VII) e (VIII), a parte de (I) è levada em (III) e (VII) e a de (II) em (IV) e (VIII); jä a bola $B_{\omega}^{2}$ é a bola com centro no ponto imprōprio contida em (IX), (X), (XI) e (XII), a parte de (I) é levada em (IX) e (XI) e a de (II) em (X) e (XII).

6) Finalmente uma bola $B_{V}^{6}$ com centro em $(0,2 \sqrt{3})$ contida em (I), (IV), (VI), (VII), (IX) e (XII). No quociente as partes de (VII), (IX) são levadas em (I) e as partes de $B_{V}^{6}$ em (IV), (VI) e (XII) na parte da bola $B_{V}^{6}$, em (II) onde $B_{V}^{6}$, é a bola com centro em $(0,-2 \sqrt{3})$ contida em (II), (III), (V), (VIII), (X) e (XI). Afirmamos que o conjunto $A_{6}$ constituido pelas partes de $B_{V}^{6}$ e $B_{V}^{6}$, em (I) e (II) é um aberto no quociente. De fato $\phi^{-1}\left(A_{6}\right)=B_{V}^{6} \cup B_{V^{\prime}}^{6}$ por continuidade.

Agora temos uma topologia no espaço das ôrbitas. 
2.2 - As örbitas e a razão dupla entres as retas que se decompõe o germe $x^{4}+a x^{2} y^{2}+y^{4}$

Façamos $x^{2}=X$ e $y^{2}=Y$, então $x^{4}+a x^{2} y^{2}+y^{4}=x^{2}+a X Y+y^{2}=$ $(X-\alpha Y)(X-\beta Y)$ onde $\alpha=\frac{-a+\sqrt{a^{2}-4}}{2}$ e $\beta=\frac{-a-\sqrt{a^{2}-4}}{2}$.

Substituindo $X, Y$ por $x$ e $y$ novamente, obtemos: $\left(x^{2}-\alpha y^{2}\right)\left(x^{2}-\beta y^{2}\right)=$

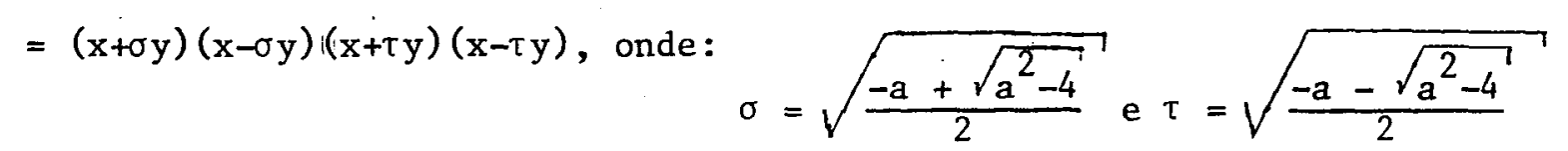

a1ém disso $\alpha \beta=1, \alpha+\beta=-\mathbf{a}, \sigma^{2}=+\alpha, \tau^{2}=+\beta$ e $(\sigma \tau)^{2}=1$. Escolhemos $\sigma$ e $\tau$ de tal forma que $\sigma \tau=1$.

Podemos pensar então em calcular a razão dupla entre as quatro re tas $x=-\sigma y ; x=\sigma y ; x=-\tau y$ e $x=\tau y$. Esta razão pode ser obtida cortando as retas pela reta $y=1$, obtemos: $(-\sigma, \sigma,-\tau, \tau)$.

$$
\begin{aligned}
d & =\operatorname{razão} \operatorname{dup} l a(-\sigma, \sigma,-\tau, \tau)=\frac{(-\sigma, \sigma,-\tau)}{(-\sigma, \sigma, \tau)}=\frac{\frac{-\tau+\sigma}{-\tau-\sigma}}{\frac{\tau+\sigma}{\tau-\sigma}}=\left(\frac{\tau-\sigma}{\tau+\sigma}\right)^{2}= \\
& =\frac{\tau^{2}-2 \sigma \tau+\sigma^{2}}{\tau^{2}+2 \sigma \tau+\sigma^{2}}, \text { então } d=\frac{-a-2}{-a+2}=\frac{a+2}{a-2} .
\end{aligned}
$$

Lembramos que permutando as retas, obtemos ao total 6 (seis) valo res para a razão dupla as quais são invariantes por difeomorfismos. Associando $a b_{1}=a$ o valor $d=\frac{a+2}{a-2}$ como valor principal observamos que fica associado $a b_{i}$ o valor $d_{i}=\frac{b_{i}+2}{b_{i}-2}$ que percorre os 6 (seis) valores da razão dupla correspondentes às permutações das retas. De fato:

A razão dupla correspondente $a b_{2}=-a$ e $d_{2}=\frac{-a+2}{-a-2}=\frac{a-2}{a+2}=\frac{1}{d}$.

A razāo dupla correspondente $a b_{3}=\frac{12-2 a}{2+a}$ é $d_{3}=\frac{\frac{12-2 a}{2+a}+2}{\frac{12-2 a}{2+a}-2}=1-d$. 
A razão dupla correspondente a $b_{4}=\frac{-12+2 a}{2+a}$ é $d_{4}=\frac{\frac{-12+2 a}{2+a}+2}{\frac{-12+2 a}{2+a}-2}=\frac{1}{1-d}$.

A razão dupla correspondente a $b_{5}=\frac{12+2 a}{2-a}$ é $d_{5}=\frac{\frac{12+2 a}{2-a}+2}{\frac{12+2 a}{2-a}-2}=\frac{d-1}{d}$.

A razão dupla correspondente $a b_{6}=\frac{-12-2 a}{2-a}$ é $d_{6}=\frac{\frac{-12-2 a}{2-a}+2}{\frac{-12-2 a}{2-a}-2}=\frac{d}{d-1}$.

Notas: 1. Vimos que se escolhermos $\tau \sigma=1 \mathrm{e}$ considerarmos todos os germes de uma mesma ôrbita, obtemos para cada germe uma razão dupla, obtida por uma permutação entre as quatro retas originais.

2. Observamos que a örbita de 0 sömente contêm mais dois pontos 6 e $-6$.

para $a=0: d=-1 ; d_{2}=-1 ; d_{3}=2 ; d_{4}=1 / 2 ; d_{5}=2$ e $d_{6}=1 / 2$.

para $a=6: d=2 ; d_{2}=1 / 2 ; d_{3}=-1 ; d_{4}=-1 ; d_{5}=1 / 2$ e $d_{6}=2$.

para $a=-6: d=1 / 2 ; d_{2}=2 ; d_{3}=1 / 2 ; d_{4}=2 ; d_{5}=-1$ e $d_{6}=-1$.

Os seis valores se reduzem portanto a três $\{-1,2,1 / 2\}$. Neste caso dizemos que $(-\sigma, \sigma,-\tau, \tau)$ forma um conjunto harmônico.

3. Os pontos $-2,2$ e $\infty$ são de uma mesma örbita que corresponde ao caso em que uma das retas tende a posição da outra.

\section{3- Outro exemplo}

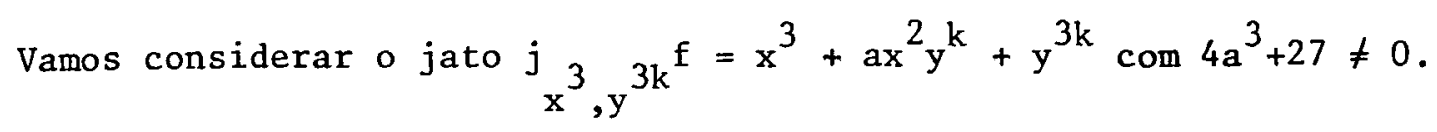
o Teorema 1.7, garante que neste caso $f \in J_{k, 0}$, cuja forma norma1 $\hat{e} x^{3}+a x^{2} y^{k}+$ $+y^{3 k}+c x y^{2 k+1}$, onde $c=c_{0}+\ldots+c_{k-3} y^{k-3}$ para $k>2$; para $k=2$, tomase $c=0$. 
ta apōs a ação de um difeomorfismo que deixa $f_{0}$ fixo a menos de germes de uma mesma örbita. Passando ao quociente pela relação "germes de uma mesma ör bita", encontraremos o espaço de órbitas. Faremos isto para o caso particu lar em que $k=2$, is.to é, o germe $x^{3}+a x^{2} y^{2}+y^{6}$.

Façamos então a mudança:

$$
\left\{\begin{array}{l}
x=\alpha X+\beta Y^{2} \\
y=\delta Y
\end{array}\right.
$$

Ficamos com:

$$
\begin{aligned}
& \left(\alpha X+\beta Y^{2}\right)^{3}+a\left(\alpha X+\beta Y^{2}\right)^{2}(\delta Y)^{2}+(\delta Y)^{6}= \\
& =\alpha^{3} X^{3}+\left(3 \alpha^{2} \beta+a \alpha^{2} \delta^{2}\right) X^{2} Y^{2}+\left(3 \alpha \beta^{2}+2 a \alpha \beta \delta^{2}\right) X Y^{4}+\left(\beta^{3}+a \beta^{2} \delta^{2}+\delta^{6}\right) Y^{6}= \\
& =x^{3}+a x^{2} y^{2}+y^{6} .
\end{aligned}
$$

Segue que:

$$
\left\{\begin{array} { l } 
{ \alpha ^ { 3 } = 1 } \\
{ 3 \alpha ^ { 2 } \beta + a \alpha ^ { 2 } \delta ^ { 2 } = b } \\
{ 3 \alpha \beta ^ { 2 } + 2 a \alpha \beta \delta ^ { 2 } = 0 } \\
{ \beta ^ { 3 } + a \beta ^ { 2 } \delta ^ { 2 } + \delta ^ { 6 } = 1 }
\end{array} \quad \left\{\begin{array}{l}
\alpha^{3}=1 \\
\alpha^{2}\left(3 \beta+a \delta^{2}\right)=b \\
\alpha \beta\left(3 \beta^{2}+2 a \delta^{2}\right)=0 \\
\beta^{3}+a \beta^{2} \delta^{2}+\delta^{6}=1
\end{array}\right.\right.
$$

10. Caso: Se $\beta=0$, em (4) $\delta^{6}=1$. Portanto:

$$
\delta=\left\{1, \frac{1+\sqrt{3} i}{2}, \frac{-1+\sqrt{3} i}{2},-1, \frac{-1-\sqrt{3} i}{2}, \frac{1-\sqrt{3} i}{2}\right\}
$$

De (1) $\alpha^{3}=1, \log \alpha \alpha=\left\{1, \frac{-1+\sqrt{3} i}{2}, \frac{-1-\sqrt{3} i}{2}\right\}$

(i) e (ii) em (2) $a \alpha^{2} \delta^{2}=b$, como $\alpha^{2} \delta^{2}=\left\{1, \frac{-1+\sqrt{3} i}{2}, \frac{-1-\sqrt{3} i}{2}\right\}$, teremos:

$$
b_{1}=a ; b_{2}=a\left(\frac{-1+\sqrt{3} i}{2}\right) ; b_{3}=a\left(\frac{-1-\sqrt{3} i}{2}\right)
$$

20. Caso: se $3 \beta+2 a \delta^{2}=0 \Rightarrow \beta=\frac{-2 a \delta^{2}}{3}$, em (4) 


$$
\begin{gathered}
\left(\frac{-2 a \delta^{2}}{3}\right)^{3}+\delta^{2}\left[a\left[\frac{-2 a \delta^{2}}{3}\right]^{2}+\delta^{4}\right]=1 \Rightarrow \\
\Rightarrow \frac{-8}{27} a^{3} \delta^{6}+\frac{4 a^{3} \delta^{6}}{9}+\delta^{6}=1 \Rightarrow \delta^{6}\left(\frac{-8 a^{3}}{27}+\frac{4 a^{3}}{9}+1\right)=1
\end{gathered}
$$

Como por hipötese $4 a^{3}+27 \neq 0$, segue que:

$$
\delta^{6}=\frac{27}{4 a^{3}+27} \Rightarrow \delta^{2}=\sqrt[3]{\frac{27}{4 a^{3}+27}}
$$

De (2) $\alpha^{2}\left[3\left(\frac{-2 a \delta^{2}}{3}\right)+a \delta^{2}\right]=b \Rightarrow-a \alpha^{2} \delta^{2}=b$

$$
b_{4}=-a \sqrt[3]{\frac{27}{4 a^{3}+27}} ; \quad b_{5}=-a \sqrt[3]{\frac{27}{4 a^{3}+27}}\left(\frac{-1+\sqrt{3} i}{2}\right) ; b_{6}=-a \sqrt[3]{\frac{27}{4 a^{3}+27}}\left(\frac{-1-\sqrt{3} i}{2}\right)
$$

Representando $x^{3}+a x^{2} y^{2}+y^{6}$ pelo ponto a do plano complexo, ob temos na Figura $B$ uma regiāo (hachuriada) que representa todas as classes de equivalências dos germes, isto $\dot{e}$, a região contèm representantes de todas as diferentes ôrbitas (sistema de representantes).

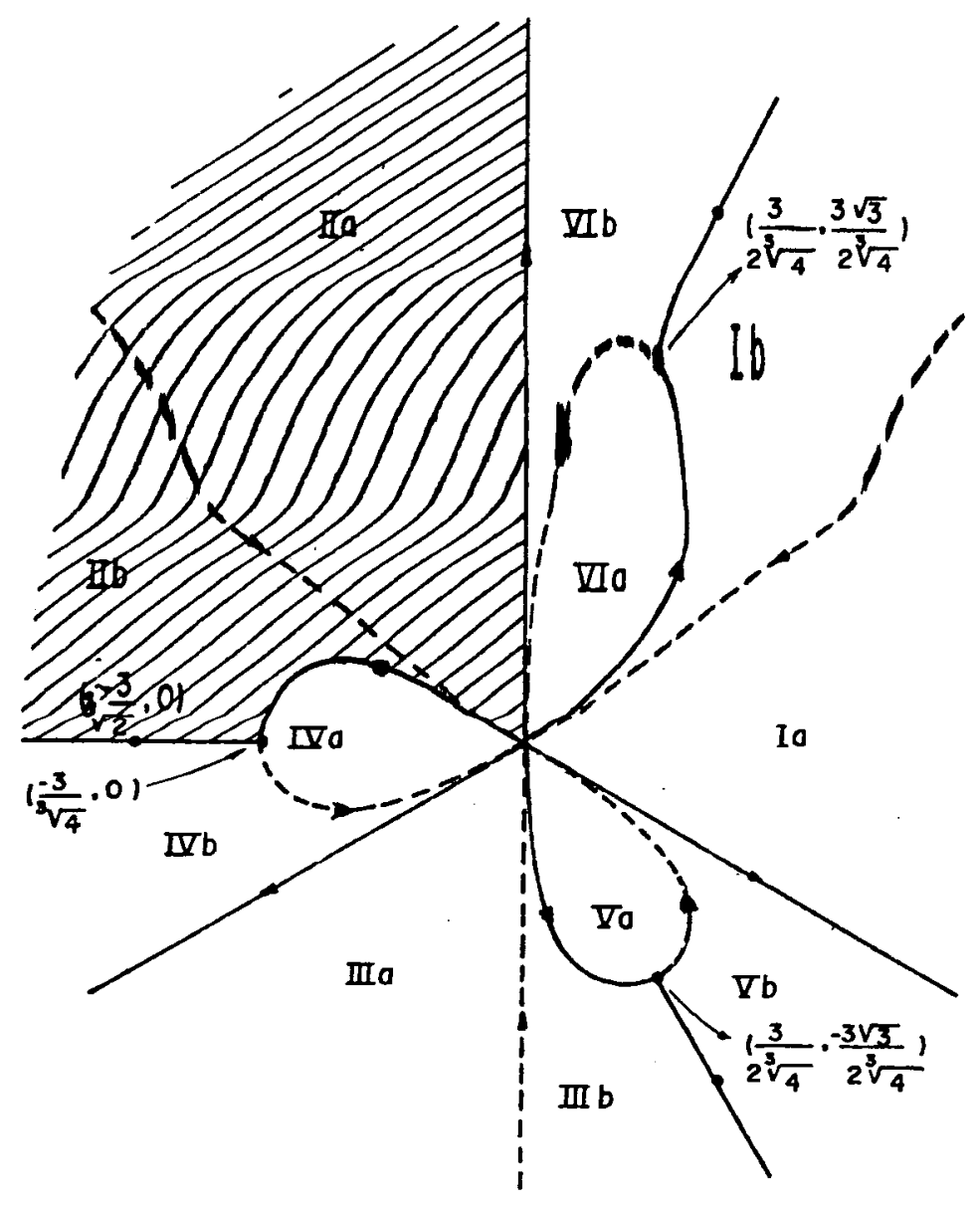


Em seguida demonstraremos este fato.

Seja $a=\lambda i$. Vamos analisar como se comporta $b_{1}, b_{2}, b_{3}, b_{4}, b_{5} e$ $b_{6} \cdot$

Temos que $: b_{1}=(0, \lambda) ; b_{2}=\left(\frac{-\sqrt{3}}{2} \lambda, \frac{-1}{2} \lambda\right) ; b_{3}=\left(\frac{\sqrt{3}}{2} \lambda ; \frac{-1}{2} \lambda\right)$

Estudaremos o comportamento de $\mathrm{b}_{4}$

$b_{4}=-\lambda \sqrt[3]{\frac{27}{27-4 \lambda^{3} i}} i=-\frac{3 \lambda}{\sqrt{27^{2}+\left(4 \lambda^{3}\right)^{2}}}\left(\cos \frac{\theta+2 k \pi}{3}+i \operatorname{sen} \frac{\theta+2 k \pi}{3}\right) i$

onde $\theta=\operatorname{arctg} \frac{4 \lambda^{3}}{27}, k=0,1,2$.

$$
\begin{aligned}
\text { Assim } b_{4} & =\frac{3 \lambda}{\sqrt{27^{2}+\left(4 \lambda^{3}\right)^{2}}} \operatorname{sen}\left(\frac{\operatorname{arctg}\left(4 \lambda^{3} / 27\right)+2 k \pi}{3}\right) \\
& -\frac{3 \lambda}{\sqrt[6]{27^{2}+\left(4 \lambda^{3}\right)^{2}}} \cos \left(\frac{\operatorname{arctg}\left(4 \lambda^{3} / 27\right)+2 k \pi}{3}\right) i
\end{aligned}
$$

*0bservação: As três raízes obtidas variando $\mathrm{k}$, nos fornecem tambêm as três raizes de $b_{5} e b_{6}$ para diferentes valores de $k$. Fixaremos um va lor de $k$ e assim obteremos $b_{4}, b_{5}$ e $b_{6}$. Se $k=0, b_{5}$ coincide com $b_{4}$ para $k=1 e \operatorname{com} b_{6}$ caso $k=2$, Antes porem vamos analisar o que nos fornecem geo metricamente essas trés raízes fazendo $\lambda$ variar.

(a) $x=\operatorname{sen}\left(\frac{\operatorname{arctg}\left(4 \lambda^{3} / 27\right)+2 k \pi}{3}\right) \frac{3 \lambda}{\sqrt[6]{27^{2}+\left(4 \lambda^{3}\right)^{2}}}$

(b) $y=-\cos \left(\frac{\operatorname{arctg}\left(4 \lambda^{3} / 27\right)+2 k \pi}{3}\right) \frac{3 \lambda}{6 \sqrt{27^{2}+\left(4 \lambda^{3}\right)^{2}}}$ 
Mas $\operatorname{tg} 3 \alpha=\frac{3 \operatorname{tg} \alpha-\operatorname{tg}^{3} \alpha}{1-3 \operatorname{tg}^{2} \alpha} \quad(* *)$
Fazendo $\alpha=\operatorname{arctg}(x / y) \Rightarrow \operatorname{tg} \alpha=x / y$

$$
\begin{aligned}
& \frac{-4 \lambda^{3}}{27}=-\operatorname{tg}\left(\operatorname { a r c t g } ( 4 \dot { \lambda } ^ { 3 } / 2 7 ) \stackrel { ( * ) } { = } \operatorname { t g } \left(3 \operatorname{arctg}(x / y)=\operatorname{tg} 3 \alpha \stackrel{(* *)}{=} \frac{3 \operatorname{tg} \alpha-\operatorname{tg}^{3} \alpha}{1-3 \operatorname{tg}^{2} \alpha}=\right.\right. \\
& \frac{3(x / y)-(x / y)^{3}}{1-3(x / y)^{3}}=\frac{x^{3}-3 x y^{2}}{3 x^{2} y-y^{3}} .
\end{aligned}
$$$$
\text { Assim: } \quad \frac{x^{3}-3 x y^{2}}{3 x^{2} y-y^{3}}=-\frac{4 \lambda^{3}}{27}
$$

Somando-se os quadrados de (a) e (b) e substituindo em (c), obte mos:

$$
\begin{aligned}
& x^{2}+y^{2}=\frac{9 \lambda^{2}}{\sqrt[3]{\left(4 \lambda^{3}\right)^{2}+27^{2}}}=9 \sqrt[3]{\frac{(27 / 4)^{2}\left(\frac{\left.-x^{3}+3 x y^{2}\right)^{2}}{3 x^{2} y-y^{3}}\right)^{2}}{\left.27^{2}\left(\frac{-x^{3}+3 x y^{2}}{3 x^{2} y-y^{3}}\right)^{2}+1\right)}}= \\
& =\frac{9}{2 \sqrt[3]{2}\left(x^{2}+y^{2}\right)} \sqrt[3]{\left(-x^{3}+3 x y^{2}\right)^{2}} \Rightarrow 2\left(x^{2}+y^{2}\right)^{2}=9\left(\frac{\left(-x^{3}+3 x y^{2}\right)^{2}}{2}\right) \Rightarrow \\
& 4\left(x^{2}+y^{2}\right)^{3}=27\left(-x^{3}+3 x y^{2}\right) \Rightarrow x^{2}+y^{2}=\frac{3}{\sqrt[3]{4}}\left(-x^{3}+3 x y^{2}\right)^{1 / 3}
\end{aligned}
$$

equaçöes (a) e (b), podemos fazer $\lambda \rightarrow \infty$ e $\lambda \rightarrow-\infty$, com $k=0,1,2$.

(i) se $k=0$, então:

$$
\begin{aligned}
& \lambda \rightarrow \infty, \text { nos fornece }\left(\frac{3}{2^{3} \sqrt{4}}, \frac{-3 \sqrt{3}}{2^{3} \sqrt{4}}\right) \\
& \lambda \rightarrow-\infty, \text { nos fornece }\left(\frac{3}{2^{3} \sqrt{4}}, \frac{3 \sqrt{3}}{2^{3} \sqrt{4}}\right)
\end{aligned}
$$

(ii) se $k=1$, então:

$$
\lambda \rightarrow \infty \quad \text {, nos fornece }\left(\frac{3}{2^{3} \sqrt{4}}, \frac{3 \sqrt{3}}{2^{3} \sqrt{4}}\right)
$$


$\lambda \rightarrow-\infty$, nos fornece $\left(\frac{-3}{\sqrt[3]{4}}, 0\right)$

(iii) se $k=2$, então:

$$
\begin{aligned}
& \lambda \rightarrow \infty, \text { nos fornece }\left(\frac{-3}{\sqrt[3]{4}}, 0\right) \\
& \lambda \rightarrow-\infty, \text { nos fornece }\left(\frac{3}{2 \sqrt[3]{4}}, \frac{-3 \sqrt{3}}{2 \sqrt[3]{4}}\right)
\end{aligned}
$$

Assim pela observação anterior, juntamente com o descrito acima, quando $\lambda$ varia de $-\infty a+\infty$ temos que $b_{4}$ percorre o trecho da rosácea de $\left(\frac{3}{2^{3} \sqrt{4}}, \frac{3 \sqrt{3}}{2}\right)$ a $\left(\frac{3}{2 \sqrt[3]{4}}, \frac{-3 \sqrt{3}}{2}\right) ; b_{5}$ percorre o trecho da rosacea de $\left(\frac{-3}{\sqrt[3]{4}}, 0\right)$ a $\left(\frac{3}{2 \sqrt[3]{4}}, \frac{3 \sqrt{3}}{2 \sqrt[3]{4}}\right) ; b_{6}$ percorre o trecho da rosacea de $\left(\frac{3}{2 \sqrt[3]{4}}, \frac{-3 \sqrt{3}}{2}\right)$ a $\left(\frac{-3}{\sqrt[3]{4}}, 0\right)$.

Os pontos interiores à rosácea satisfazem a desigualdade:

$$
x^{2}+y^{2}<\frac{3}{\sqrt[3]{4}}\left(-x^{3}+3 x y^{2}\right)^{1 / 3} \text { e os exteriores a desigualdade com } 0
$$

sinal maior.

Consideremos as regiões em $\mathbf{C}^{1}$ dadas por:

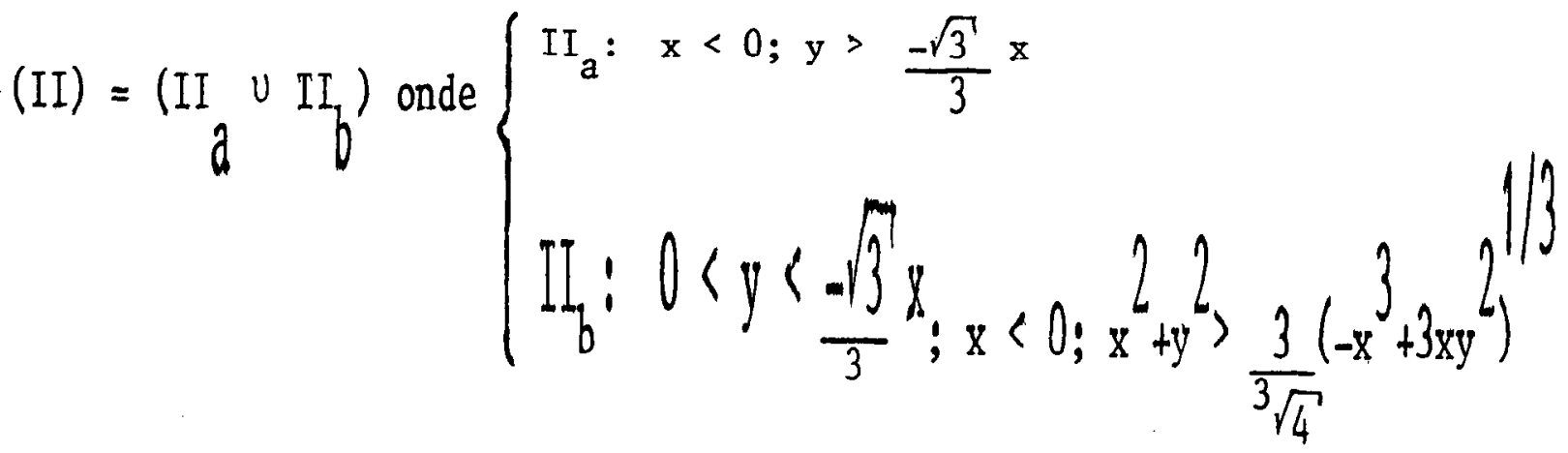

$$
\begin{aligned}
& (I I I)=\left(\operatorname{III}_{a} \cup \operatorname{III}_{b}\right) \text { onde }\left\{\begin{array}{l}
\operatorname{III}_{a}: y<\frac{\sqrt{3}}{3} x ; x<0 \\
\operatorname{III}_{b}: x>0 ; y<-\sqrt{3} x ; x^{2}+y^{2}<\frac{3}{3 \sqrt{h}}\left(-x^{3}+3 x y^{2}\right)^{1 / 3}
\end{array}\right.
\end{aligned}
$$




$$
\begin{aligned}
& (V I)=\left(V I_{a} \cup V_{B}\right) \text { onde }\left\{\begin{array}{l}
V_{a}: x<0 ; x^{2}+y^{2}<\frac{3}{\sqrt[3]{4}}\left(-x^{3}+3 x y^{2}\right)^{1 / 3} \\
V_{b}: x<0 ; \frac{\sqrt{3}}{3} x<y<0 ; x^{2}+y^{2}>\frac{3}{3 \sqrt{4}}\left(-x^{3}+3 x y^{2}\right)^{1 / 3}
\end{array}\right. \\
& (I V)=\left(I V_{a} \cup I V_{b}\right) \text { onde }\left\{\begin{array}{l}
I V_{a}: x>0 ; y>0 ; x^{2}+y^{2}<\frac{3}{\sqrt[3]{4}}\left(-x^{3}+3 x y^{2}\right)^{1 / 3} \\
I V_{b}: y>-\sqrt{3} x ; y<\frac{-\sqrt{3}}{3} x ; x^{2}+y^{2}>\frac{3}{3 \sqrt{4}}\left(-x^{3}+3 x y^{2}\right)^{1 / 3}
\end{array}\right. \\
& (V)=\left(V_{z} \cup V_{b}\right) \text { onde }\left\{\begin{array}{l}
V_{a}: x>0 ; y>0 ; x^{2}+y^{2}<\frac{3}{\sqrt[3]{4}}\left(-x^{3}+3 x y^{2}\right)^{1 / 3} \\
V_{b}: x>0 ; y>\sqrt{3} x ; x^{2}+y^{2}>\frac{3}{3 \sqrt{4}}\left(-x^{3}+3 x y^{2}\right)^{1 / 3^{\prime}}
\end{array}\right.
\end{aligned}
$$

Mostraremos que os pontos de $I_{a}$ são levados em:

$I_{a}$ por $b_{2}$
$I I I_{a}$ por $b_{3}$
$I_{a}$ por $b_{4}$
$V_{a}$ por $b_{5}$
$V_{a} \operatorname{por~} b_{6}$

De fato: $\quad 1$ rotação de $120^{\circ}$

2 rotação de $240^{\circ}$

3 (i) $\begin{cases}y=\frac{\sqrt{3}}{3} \mathrm{x} & \text { è levado por } b_{4} \text { nas partes tracejadas } \\ v>n & \text { do }{ }^{2}+2^{2}-3, \ldots 3 ., 2,1 / 3\end{cases}$ 
Assim por continuidade $I_{a}$ é levado por $b_{4}$ em $I_{a}$

4 Aplicar $b_{5}$ em pontos de $I_{a}$ é equivalente a aplicar $b_{4}$ em segui da aplicar $b_{2}$. Assim de 3 e 1 temos que $I_{a}$ è levado em

$V_{a}$ por $b_{5^{\prime}}$

5 Aplicar $b_{6}$ em pontos de $I_{a}$ é equivalente a fazer a composição $\mathrm{b}_{3} \circ \mathrm{b}_{4}$. Assim de 3 e 2 temos que $\mathrm{I}_{\mathrm{a}}$ ë levado em $\mathrm{VI}_{\mathrm{a}}$ por $b_{6} \cdot$

Alem disso os pontos de $I_{b}$ são levados em :

$\mathrm{II}_{\mathrm{b}}$ por $\mathrm{b}_{2} \quad 1^{\prime}$

$\operatorname{III}_{\mathrm{b}}$ por $\mathrm{b}_{3} \quad 2^{\prime}$

$I V_{b}$ por $b_{4} \quad 3^{\prime}$

$V_{b}$ por $b_{5} \quad 4^{\prime}$

$\mathrm{VI}_{\mathrm{b}}$ por $\mathrm{b}_{6} \quad 5^{\prime}$

De fato: $1^{\prime}$ rotação de $120^{\circ}$

$2^{\prime}$ rotação de $240^{\circ}$

$3^{\prime}$ jà vimos em 3 (i). Mostremos

(iv) as partes de traço contínuo da rosäcea que é levada por b, coincidem

(v) a semi-reta $\left\{y=\sqrt{3} x ; x>\frac{3}{2 \sqrt[3]{4}}\right\}$ e levada por $b_{4}$ em 
damental de representantes.

Para obtermos o espaço de órbitas observemos que podemos identificar a semi-reta $\{x=0, y \geqq 0\}$ com $\left\{x^{2}+y^{2}=\frac{3}{\sqrt[3]{4}}\left(-x^{3}+3 x y^{2}\right)^{\uparrow / 3}, x \leqq 0, y \geqq 0\right\}$ no sentido crescente e $\left\{y=0 ; \frac{-3}{\sqrt[3]{2}} \leqq x<\frac{-3}{\sqrt[3]{4}}\right\}$ com $\left\{y=0,-\infty<x \leqq \frac{-3}{\sqrt[3]{2}}\right\}$ no sen tido decrescente.

Fazendo estas identificações obtemos como espaço de örbitas $\mathrm{s}^{2}$. De maneira anāloga ao exemplo 2.1 mostra-se que o quociente é homeoforfo a $\mathrm{s}^{2}$.

$\$ 3$ - Comportamento da parte $f^{\prime}$ de uma função semiquasehomogênea $f=f_{0}+f^{\prime}$ após difeomorfismo que deixa $f_{0}$ fixo a menos de germes de uma mesma ôrbita.

Consideraremos inicialmente o exemplo estudado no ítem 2.3 , isto é, $\mathrm{x}^{3}+a \mathrm{x}^{2} \mathrm{y}^{\mathrm{k}}+\mathrm{y}^{3 \mathrm{k}}+\mathrm{cxy} \mathrm{y}^{3 \mathrm{k}+1}$ onde $c=c_{0}+\ldots+c_{k-3} \mathrm{y}^{\mathrm{k}-3}$

Neste exemplo estudaremos as mudanças de coordenadas que deixam $f_{0}$ fixo. Em seguida faremos um estudo utilizando uma mudança que deixa $f_{o}$ fixo a menos de germes da mesma órbita.

$$
\begin{aligned}
& \text { Começaremos com o caso } k=3 \text {, isto } \dot{e} \text {, o germe } x^{3}+a x^{2} y^{3}+y^{9}+c x y^{7} \text {. } \\
& \text { Façamos a mudança: }\left\{\begin{array}{l}
x=\alpha X+\beta Y^{3} \\
y=\delta Y
\end{array} \text { em } f_{0}\right. \text {. Obtemos então: } \\
& \left(\alpha X+\beta Y^{3}\right)^{3}+a\left(\alpha X+\beta Y^{3}\right)^{2}(\delta Y)^{3}+(\delta Y)^{9}= \\
& =\alpha^{3} X^{3}+\left(3 \alpha^{2} \beta+a \alpha^{2} \delta^{3}\right) X^{2} Y^{3}+\left(3 \alpha \beta^{2}+2 a \alpha \beta \delta^{3}\right) X Y^{6}+\left(\beta^{3}+\beta^{2} \delta^{3} a+\delta^{9}\right) Y^{9} \text {. }
\end{aligned}
$$

$$
\alpha^{3}=1
$$




$$
\begin{aligned}
& 3 \alpha^{2} \beta+a_{\alpha}{ }^{2} \delta^{3}=a \Rightarrow \alpha^{2}\left(3 \beta+a \delta^{3}\right)=a \\
& 3 \alpha \beta^{2}+2 \mathrm{a}_{\alpha \beta \delta^{3}}=0 \Rightarrow \alpha \beta\left(3 \beta+2 \mathrm{a} \delta^{3}\right)=0 \\
& \beta^{3}+a \beta^{2} \delta^{3}+\delta^{9}=1
\end{aligned}
$$

10. Caso: $\beta=0$

$$
\begin{aligned}
& \text { em }(4) \Rightarrow \delta^{9}=1 \\
& \text { em }(2) \Rightarrow a^{2} \delta^{3}=1
\end{aligned}
$$

Sejam: $\varepsilon_{i}(i=0,1,2)$ as raizes triplas da unidade

$$
\phi_{j}(j=0,1,2, \ldots, 8) \text { as raízes nonas da unidade }
$$

Assim de (1) e (5) a dança será $\left\{\begin{array}{l}x=\varepsilon_{i} X \\ y=\phi_{j} Y\end{array}\right.$

Logo $f^{\prime}$ fica da forma: $c_{0} x^{7}=c_{0}\left(\varepsilon_{i} X\right)\left(\phi_{j} Y\right)^{7}=c_{o} \varepsilon_{i} \phi_{j}^{7} X Y^{7}$. Mas temos a restrição (6), isto $\dot{e}, \varepsilon_{i}{ }^{2}{ }_{j}^{3}=1$ ou seja $2.3 i+3 j \equiv 0 \bmod 9$ que nos fornece :

\begin{tabular}{|llllllllll|}
\hline $\mathrm{i}$ & 0 & 1 & 2 & 0 & 1 & 2 & 0 & 1 & 2 \\
\hline $\mathrm{j}$ & 0 & 1 & 2 & 3 & 4 & 5 & 6 & 7 & 8 \\
\hline
\end{tabular}

TABELA 1 Colocamos $\varepsilon_{i} \phi_{j}^{7}=\phi_{h}$, portanto $3 i+7 j \equiv h \bmod 9$, donde

\begin{tabular}{|llllllllll|}
\hline $\mathrm{i}$ & 0 & 1 & 2 & 0 & 1 & 2 & 0 & 1 & 2 \\
\hline $\mathrm{j}$ & 0 & 1 & 2 & 3 & 4 & 5 & 6 & 7 & 8 \\
\hline $\mathrm{h}$ & 0 & 1 & 2 & 3 & 4 & 5 & 6 & 7 & 8 \\
\hline
\end{tabular}

Assim o novo $f^{\prime}$ é da forma $c_{o} \phi_{j} X Y^{7}, \operatorname{com} j=0,1, \ldots, 8$.

20. Caso: $3 \beta+2 a \delta^{3}=0$

Então $\beta=\frac{-2}{3} a \delta^{3}$ em $(4) \Rightarrow \frac{-8}{27} a^{3} \delta^{9}+\frac{4}{9} a^{3} \delta^{6} \delta^{3}+\delta^{9}=1$ 
63

$$
\begin{aligned}
& \text { fazendo } \beta=\frac{-2}{3} \mathrm{a} \delta^{3} \cdot \text { em }(2) \text {, obtemos: } \\
& \qquad \alpha^{2}\left(-2 \mathrm{a} \delta^{3}+\mathrm{a} \delta^{3}\right)=\mathrm{a} \Rightarrow \alpha^{2} \delta^{3}=-1
\end{aligned}
$$

De (1) $\alpha=\varepsilon_{i}(i=0,1,2)$ em (8), segue que:

$$
\begin{aligned}
& \varepsilon_{i}^{2} \delta^{3}=-1 \Rightarrow \delta^{3}=-\varepsilon_{i} \text { substituindo em (7) obtemos : } \\
& \delta^{9}=-1 \text { e } \frac{27}{4 a^{3}+27}=-1 \Rightarrow \delta^{9}=-1 \text { e a }=\frac{-3}{\sqrt[3]{2}} \varepsilon_{l} \quad(1=0,1,2)
\end{aligned}
$$

Chamemos de $\psi_{j}(j=0,1,2, \ldots, 8)$ as raízes nonas de -1 . Segue que

$$
\beta=\frac{-2}{3}\left(\frac{-3}{\sqrt[3]{2}} \varepsilon_{i}\right)\left(\psi_{j}\right)^{3}=\frac{-2}{\sqrt[3]{2}} \varepsilon_{i} \psi_{j}^{3} \text {. }
$$

Assim a mudança é dada por:

$$
\left\{\begin{array}{l}
x=\varepsilon_{i} X-\frac{2}{\sqrt[3]{2}} \varepsilon_{j} \psi_{j}^{3} Y^{3} \\
y=\psi_{j} Y
\end{array}\right.
$$

Vamos então calcular $f^{\prime}$.

$$
c_{0} x^{7}=c_{0}\left(\varepsilon_{i} X-\frac{2}{\sqrt[3]{2}} \varepsilon_{i} \psi_{j}^{3} Y^{3}\right)\left(\psi_{j} Y\right)^{7}=c_{o} \varepsilon_{i} \psi_{j}^{7} X Y^{7}-\frac{2}{\sqrt[3]{2}} c_{0} \varepsilon_{l} \psi_{j}^{10}{ }^{10}
$$

Mas, observemos que:

$$
f_{0}=X^{3}-\frac{3}{\sqrt[3]{2}} \varepsilon X^{2} Y^{3}+Y^{9} \text {. Portanto: }
$$

$$
\frac{\partial f_{o}}{\partial X}=3 X^{2}-\frac{6}{\sqrt[3]{2}} \varepsilon_{l} X Y^{3} \quad \text { e } \quad \frac{\partial f_{o}}{\partial Y}=\frac{-9}{\sqrt[3]{2}} \varepsilon_{l} X^{2} Y^{2}+9 Y^{8}
$$

$$
\begin{aligned}
& \text { e portanto: } Y^{10}=\frac{1}{9} Y^{2} \frac{\partial f_{o}}{\partial Y}+\frac{1}{3 \sqrt[3]{2}} Y^{4} \varepsilon 1 \frac{\partial f_{o}}{\partial X}+\frac{2}{\sqrt[3]{4}} \varepsilon I^{2} X Y^{7} \\
& \text { em }(*) c_{o} X^{7}=c_{o} \varepsilon_{i} \psi_{j}{ }^{7} X Y^{7}-\left(2 /^{3} \sqrt{2}\right) c_{o} \varepsilon_{l}{ }^{10} \cdot\left(2 /^{3} \sqrt{4}\right) \varepsilon_{q}{ }^{2} X Y^{7}+\text { He }
\end{aligned}
$$


Mostramos no 19 Caso que $\varepsilon_{i} \phi_{j}^{7}=\phi_{j}$, de modo análogo mostra-se que $\varepsilon_{i} \phi^{7}=\psi_{j}$
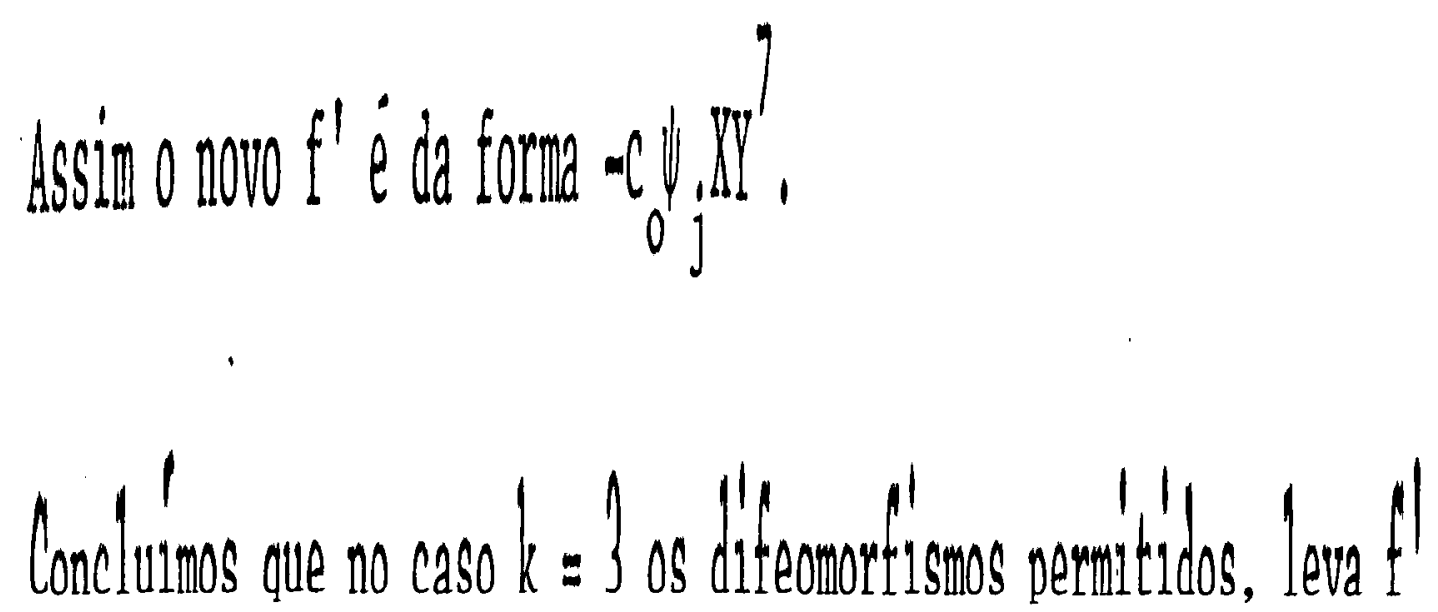

em 18 elementos de uma mesma örbita, a saber, $c_{0} \phi_{j}$ e $c_{0} \psi_{j}$ onde $\phi_{j}$ são as raizes nonas da unidade e $\psi_{j}$ são as raizes nonas de -1 .

Façamos agora 0 mesmo estudo para o caso em que $\mathrm{k} \dot{\mathrm{e}}$ arbiträrio. A

funçào è da forma:

$x^{3}+a x^{3} y^{k}+y^{3 k}+c_{0} x y^{2 k+1}+c_{1} x y^{2 k+2}+\ldots+c_{k-3} x y^{3 k-2}$ para $k>2$.

A mudança que faremos $\dot{e}$ do tipo:

$\left\{\begin{array}{l}x=\alpha X+\beta Y^{k} \\ y=\delta Y\end{array}\right.$

Obteremos o seguinte sistema que denotaremos por $S$ : 
Então a parte semiquasehomogênea fica:

$c_{o} \varepsilon_{i} j^{2 k+1} X Y^{2 k+1}+c_{1} \varepsilon_{i} j^{2 k+2} X Y^{2 k+2}+\ldots+c_{k-3} \varepsilon_{i} \phi_{j}^{3 k-2} X Y Y^{3 k-2}$.

Mas temos a restrição (6) $\varepsilon_{j}{ }^{2}{ }_{i j}{ }^{k}=1$ que nos leva a $2 i+j \equiv 0 \bmod 3$

que implicará numa tabela análoga a Tabela 1.

Assim (a) $\varepsilon_{i}^{\phi_{j}}{ }^{2 k+1}=\phi_{h_{1}}$ ou seja k.i $+(2 k+1) j \equiv h_{1}$ mod $3 k$ nos dan do:

\begin{tabular}{|lllllllll|}
\hline$i$ & 0 & 1 & 2 & 0 & 1 & 2 & $\ldots$ & 2 \\
\hline$j$ & 0 & 1 & 2 & 3 & 4 & 5 & $\ldots$ & $3 k-1$ \\
\hline$h_{1}$ & 0 & 1 & 2 & 3 & 4 & 5 & $\ldots$ & $3 k-1$ \\
\hline
\end{tabular}

$$
\text { Portanto } \varepsilon_{\mathbf{i}^{\phi}}{ }^{2 \mathrm{k}+1}=\phi_{j}
$$

(b) $\varepsilon_{i} \phi_{j}{ }^{2 k+2}=\varepsilon_{i} \phi_{j}{ }^{2 k+1} \phi_{j} \stackrel{\text { (a) }}{=} \phi_{j}{ }^{2}$.

$(k-2) \varepsilon_{i^{\phi}}{ }^{3 k-2}=\varepsilon_{i_{j}}{ }^{2 k+1} \phi_{j}{ }^{k-3}=\phi_{j}{ }_{j}{ }^{k-3}=\phi_{j}{ }^{k-2}$.

Assim apōs o difeomorfismo obtemos o novo $f^{\prime}$ que serâ:

$c_{0} \phi_{j} X Y^{2 k+1}+c_{1} \phi_{j}{ }^{2} X Y^{2 k+2}+\ldots+c_{k-3} \phi_{j}^{k-2} X Y^{3 k-2}$.

20. Caso: $\quad 3 \beta+2 \mathrm{a} \delta^{\mathrm{k}}=0$

$$
\text { Então } \begin{aligned}
\beta=\frac{-2}{3} a \delta^{3} \text { que em (4) nos dâ } \delta^{3 k}=\frac{27}{4 a^{3}+27} \\
\text { em (2) nos dá } \alpha_{\alpha}^{2} \delta^{k}=-1
\end{aligned}
$$

de (1) $\alpha=\varepsilon_{i}(i=0,1,2)$ em $(8) \Rightarrow \varepsilon_{i}^{2} \delta^{k}=-1 \Rightarrow \delta^{k}=-\varepsilon_{i}$ que substituindo em (7) nos fornece $\delta^{3 \mathrm{k}}=-1$ e $\frac{27}{3}=-1$ ou seja: 


$$
\begin{aligned}
& B=-\frac{2}{3 \sqrt{2}} \varepsilon_{\ell} \psi_{j}^{k} \text { e a mudança é dada por : } \\
& \left\{\begin{array}{l}
x=\varepsilon_{i} X-\frac{2}{\sqrt[3]{2}} \varepsilon_{j}^{\psi}{ }_{j}^{k} Y^{k} \\
y=\psi_{j} Y
\end{array}\right.
\end{aligned}
$$

Vamos estudar o comportamento da parte semiquasehomogénea após a mudança acima:

$$
\begin{aligned}
& c_{o}\left(\varepsilon_{i} X-\frac{2}{3 \sqrt{2}} \varepsilon_{l} \psi_{j}^{k}{ }^{k}\right)\left(\psi_{j} Y\right)^{2 k+1}+c_{1}\left(\varepsilon_{i} X-\frac{2}{3 \sqrt{2}} \varepsilon_{i} \psi_{j}^{k}{ }^{k}\right)\left(\psi_{j} Y\right)^{2 k+2}+\ldots+ \\
& c_{k-3}\left(\varepsilon_{i} X-\frac{2}{3 \sqrt{2}} \varepsilon_{q}{ }^{\psi}{ }_{j}^{k} Y^{k}\right)(\psi j Y)^{3 k-2}= \\
& =c_{0} \varepsilon_{i} \psi^{2 k+1} X Y^{2 k+1}-\frac{2}{3 \sqrt{2}} c_{0} \varepsilon_{l} \psi^{3 k+1} Y^{3 k+1}+c_{1} \varepsilon_{i} \psi_{j}^{2 k+2} X Y^{2 k+2}- \\
& -\frac{2}{3 \sqrt{2}} c_{0} \varepsilon_{l}{ }^{\psi}{ }^{3 k+2} Y^{3 k+2}+\ldots+c_{k-3} \varepsilon_{i}^{\psi} j^{3 k-2} X Y^{3 k-2}-\frac{2}{3 \sqrt{2}} c_{k-3} \varepsilon^{\psi} q_{j}^{4 k-2} Y^{4 k-2}(* *)
\end{aligned}
$$

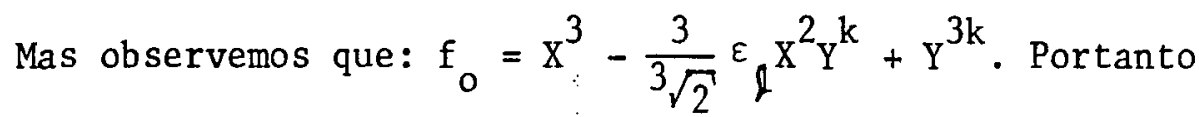

$$
\partial_{10}=3 X^{2}-\frac{6}{3 \sqrt{2}} \varepsilon_{1} X Y^{k} \quad e \partial_{2} f=-\frac{3 k}{3 \sqrt{2}} \varepsilon^{X^{2} Y^{k-1}+3 k Y^{3 k-1}}
$$

Então:

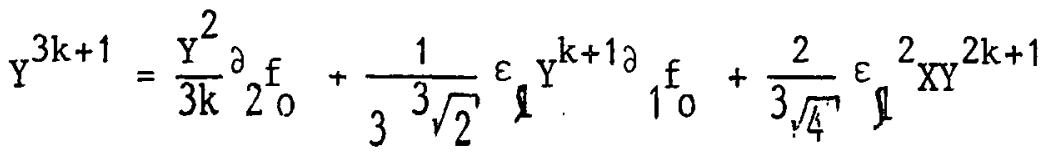

$$
\begin{aligned}
& y^{3 k+2}=\frac{y^{3}}{3 k} 2_{20}+\frac{1}{3 \sqrt{2}} \varepsilon y^{k+2} j_{1} f_{0}+\frac{2}{3 \sqrt{4}} \varepsilon_{1} 2 x y^{2 k+2} \\
& \mathrm{Y}^{4 \mathrm{k}-2}=\frac{\mathrm{Y}^{\mathrm{k}-1}}{3 \mathrm{k}} \partial_{2 \mathrm{o}}+\frac{1}{3 \sqrt[3]{2}} \varepsilon_{\ell} \mathrm{Y}^{2 \mathrm{k}-2} \partial_{1 \mathrm{o}}+\frac{2}{3 \sqrt{4}} \varepsilon_{\ell}{ }^{2} \mathrm{XY} \mathrm{Y}^{3 \mathrm{k}-2}
\end{aligned}
$$

Substituindo em $(* *)$ os $f^{\prime}$ resultantes são da forma: 


$$
\begin{aligned}
& +\ldots+c_{k-3}{ }_{i} \psi_{j}^{3 k-2} X Y^{3 k-2}-\frac{2}{\sqrt[3]{2}} c_{k-3} \varepsilon_{\ell} \psi_{j}^{4 k-2} \cdot \frac{2}{\sqrt[3]{4}} \varepsilon_{\ell}{ }^{2} X Y^{3 k-2}= \\
& =-c_{o} \psi_{l} X Y^{2 k+1}-c_{1} \psi_{l}{ }^{2} X Y^{2 k+2}-\ldots-c_{k-3} \psi^{k-2} X Y Y^{3 k-2} .
\end{aligned}
$$

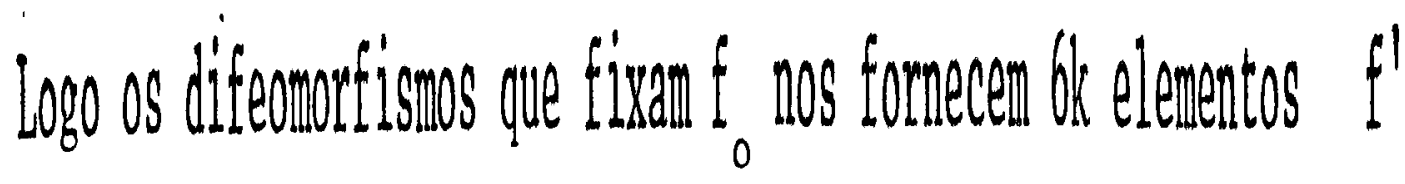
numa mesma örbita.

Um fato que nos chama a atenção é que quando fixamos o parāmetro a os ünicos valores possiveis para a são $a=-\frac{3}{\sqrt[3]{2}} \varepsilon_{j}(j=0,1,2)$ que são oontos onde aparecem apenas très elementos na mesma örbita, como visto em 2.3.

Vamos agora, estudar o comportamento da parte $\mathrm{f}^{\prime}$ quando aplicamos difeomorfismos que deixam $f_{0}$ fixo a menos dos elementos de uma mesma örbita.

Estudaremos o caso $\mathrm{k}$ arbiträrio.

Observamos que a única alteração que ocorre no sistema $S$ após a mu dança efetuada é na equação (2), ele fica agora:

$$
\begin{aligned}
\alpha^{3} & =1 \\
\alpha^{2}\left(3 \beta+a \delta^{k}\right) & =b \\
\alpha \beta\left(3 \beta+2 a \delta^{k}\right) & =0 \\
\beta^{3}+a \beta^{2} \delta^{k}+\delta^{3 k} & =1
\end{aligned}
$$

Em 2.3 vimos que para $k=2$, existia no mäximo seis elementos em cada órbita. No caso geral isto também ocorre, como mostraremos a seguir.

Caso 1: $\beta=0$

$$
\text { Temos então: }\left\{\begin{array}{l}
\alpha=\varepsilon_{i}(i=0,1,2) \\
\delta^{3 k}=1 \Rightarrow \delta=\phi_{j}(j=0,1,2, \ldots, 3 k-1)
\end{array}\right.
$$

em (2') $a \varepsilon_{i}{ }^{2} \phi_{j}^{k}=b \Rightarrow b_{1+1}=a \varepsilon_{q}(l=0,1,2)$

Caso 2: $R=-(2 / 3) a \delta^{k}$ 
em (4) $\delta^{3 k}=\frac{27}{4 a^{3}+27}$, em (2') $-a^{2} \delta^{k}=b$, donde obtemos os mes mos $b_{4}, b_{5}$ e $b_{6}$ de 2.3 .

10. Caso: $\beta=0$

A mudança neste caso é $\left\{\begin{array}{l}x=\varepsilon_{i} X \\ y=\phi_{j} Y\end{array}\right.$

A parte semiquasehomogénea é dada por:

$c_{o} \varepsilon_{i}{ }^{2 k+1} X Y^{2 k+1}+c_{1} \varepsilon_{i}{ }^{2 k}{ }^{2 k+2} X Y^{2 k+2}+\ldots+c_{k-3} \varepsilon_{i} j^{3 k-2} X Y{ }^{3 k-2}$.

Temos de (2') que $a \varepsilon_{i}^{2} \phi_{j}^{k}=b=a \varepsilon_{l} \Rightarrow \varepsilon_{i}{ }^{2} \phi_{j}^{k}=\varepsilon_{l}$

Assim existem $9 k f^{\prime}$ distintos numa mesma órbita.

20. Caso: $\beta=-\frac{2}{3} a \delta^{k}$

$\psi_{m}(m=0,1,2, \ldots, 3 k-1)$ são as raízes $3 k-e ́ s i m a s$ de $\frac{27}{4 a^{3}+27}$

A mudança neste caso é então:

$\left\{\begin{array}{l}x=\varepsilon_{i} X-\frac{2}{3} a \psi_{m}^{k}{ }^{k} \\ y=\psi_{m} Y\end{array}\right.$

Vamos estudar o comportamento da parte semiquasehomogênea após a mudança acima.

$$
\begin{aligned}
& c_{o}\left(\varepsilon_{i} X-\frac{2}{3} a \psi_{m}{ }^{k} Y^{k}\right)\left(\psi_{m} Y\right)^{2 k+1}+c_{1}\left(\varepsilon_{i} X-\frac{2}{3} a \psi_{m}{ }^{k} Y^{k}\right)\left(\psi_{m} Y\right)^{2 k+2}+\ldots+ \\
& +c_{k-3}\left(\varepsilon_{i} X-\frac{2}{3} a \psi_{m}{ }^{k} Y^{k}\right)\left(\psi_{m} Y\right)^{3 k-2}=c_{o} \varepsilon_{i} \psi_{m}^{2 k+1} X Y^{2 k+1}-\frac{2}{3} c_{o} a \psi_{m}^{3 k+1} Y^{3 k+1}+ \\
& +c_{i} \varepsilon_{i} \psi_{m}^{2 k+2} X Y^{2 k+2}-\frac{2}{3} c_{1} a \psi_{m}^{3 k+2} Y^{3 k+2}+\ldots+c_{k-3} \varepsilon_{i} \psi_{m}^{3 k-2} X Y^{3 k-2}- \\
& -\frac{2}{3} c_{k-3} a \psi_{m}^{4 k-2} Y^{4 k-2}(* * *)
\end{aligned}
$$


Mas observemos que $f_{0}=x^{3}+b X x^{k}+y^{3 k}$. Portanto:

$$
\begin{aligned}
& \frac{\partial f_{o}}{\partial X}=3 X^{2}+2 b X Y^{k} \quad e \frac{\partial f_{o}}{\partial Y}=k b X^{2} Y^{k-1}+3 k Y^{3 k-1}
\end{aligned}
$$

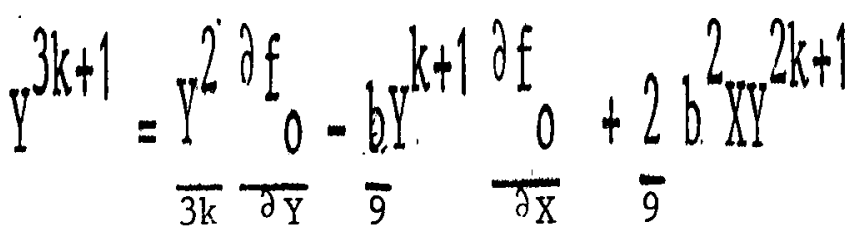

$$
\begin{aligned}
& y^{3 k+2}=\frac{Y^{3}}{3 k} \frac{\partial f_{o}}{\partial Y}-\frac{b}{9} Y^{k+2} \frac{\partial f_{o}}{\partial X}+\frac{2}{9} b^{2} X Y^{2 k+2} \\
& Y^{4 k-2}=\frac{Y^{k-1}}{3 k} \frac{\partial f}{\partial Y}-\frac{b}{9} Y^{2 k-2} \frac{\partial f_{o}}{\partial X}+\frac{2}{9} b^{2} X Y^{3 k-2}
\end{aligned}
$$

Substituindo em $(* * *)$ obtemos os possiveis $f^{\prime}$

$$
\begin{aligned}
& c_{o} \varepsilon_{i} \psi_{m}^{2 k+1} X Y^{2 k+1}-\frac{2}{3} c_{o} a \psi_{m}^{3 k+1} \cdot \frac{2}{9} b^{2} X Y^{2 k+1}+ \\
& +c_{1} \varepsilon_{i} \psi_{m}^{2 k+2} X Y^{2 k+2}-\frac{2}{3} c_{1} a \psi_{m}^{3 k+2} \cdot \frac{2}{9} b^{2} X Y^{2 k+2}+\ldots+ \\
& +c_{k-3} \varepsilon_{i} \psi_{m}^{3 k-2} X Y^{3 k-2}-\frac{2}{3} c_{k-3}{ }^{a \psi_{m}}{ }^{4 k-2} \cdot \frac{2}{9} b^{2} X Y^{3 k-2}+\pi
\end{aligned}
$$

De (2') $-\mathrm{a}_{i}{ }_{i}{ }_{\mathrm{m}}^{\mathrm{k}}=\mathrm{b} \Rightarrow \mathrm{b}^{2}=a^{2} \varepsilon_{i} \psi_{\mathrm{m}}^{2 k}$ substituindo em (f) obtemos:

$c_{0} \varepsilon_{i} \psi^{2 k+1} X Y^{2 k+1}-\frac{4}{9} c_{0} a \psi_{m}^{3 k+1}\left(a^{2} \varepsilon_{i} \psi_{m}^{2 k}\right) X Y^{2 k+1}+$

$+c_{1} \varepsilon_{i} \psi^{2 k+2} X Y^{2 k+2}-\frac{4}{9} c_{0} a \psi_{m}^{3 k+2}\left(a^{2} \varepsilon_{i m}{ }^{2 k}\right) X Y^{2 k+2}+\ldots+$

$+c_{k-3} \varepsilon_{i} \psi_{m}^{3 k-2} X Y Y^{3 k-2}-\frac{4}{9} c_{o} a \psi_{m}^{4 k-2}\left(a^{2} \varepsilon_{i} \psi_{m}^{2 k}\right) X Y=$

$c_{0} \varepsilon^{\psi_{m}}{ }^{2 k+1}\left(1-\frac{4}{9} a^{3}\right) X Y^{2 k+1}+c_{1} \varepsilon_{i}{ }^{2 k}{ }^{2 k+2}\left(1-\frac{4}{9} a^{3}\right) X Y^{2 k+2}+\ldots+$

$+c_{k-3}{ }^{\varepsilon} i_{m}^{4 k-2}\left(1-\frac{4}{9} a^{3}\right) X Y Y^{3 k-2}$. 
18k $\mathrm{f}^{\prime}$ distintos com a aplicação de difeomorfismos que fixa $\mathrm{f}_{0}$ a menos de germes da mesma örbita.

Faremos agora um estudo semelhante para

$\eta_{i, 0}: x^{3} y+a x^{2} y^{i+2}+y^{3 i+4}+c x y^{2 i+4}$, onde $i>0 e \quad c=c_{0}+\ldots+c_{i-1} y^{i-1}$.

Esta forma normal surge do teorema de classificação abaixo, cuja demonstração é anāloga a do Teorema 1.7 .

$$
j_{x^{3} y, y^{3 p+1}}{ }^{f}=y\left(x^{3}+b x^{2} y^{p}+y^{3 p}\right), 4 b^{3}+27 \neq 0 \Rightarrow f \in z_{p-1,0} .
$$

Para este exemplo faremos uma mudança de coordenadas que deixa $f_{0}$ fixo. Inicialmente faremos o caso $i=1$, para depois generalizar para $i=k$. 19 Caso $(i=1)$ : Para este caso temos a forma:

$$
\begin{gathered}
x^{3} y+a x^{2} y^{3}+y^{7}+c_{0} x y^{6} \\
f_{0}=x^{3} y+a x^{2} y^{3}+y^{7} \\
\partial_{1} f_{0}=3 x^{2} y+2 a x y^{3} \\
\partial_{2} f_{0}=3 a x^{2} y^{2}+7 y^{6}
\end{gathered}
$$

Façamos inicialmente a substituição: $\left\{\begin{array}{l}x=\alpha X \\ y=\delta Y\end{array}\right.$ em f obtemos en-

tão:

$$
\begin{aligned}
& \alpha^{3} \delta X^{3} Y+a \alpha^{2} \delta^{3} X^{2} Y^{3}+\delta Y^{7} \text {. Queremos } f_{o} \text { fixo, assim: } \\
& \alpha^{3} \delta=1 \\
& a \alpha^{2} \delta^{3}=a \Rightarrow \alpha^{2} \delta^{3}=1 \\
& .7
\end{aligned}
$$


Chamemos $\varepsilon_{1}$ a primeira raiz sétima da unidade e façamos $a=\varepsilon_{1}^{b}$ e $\delta=\varepsilon_{i}$.

de (1) $\varepsilon_{q}^{3 b} \cdot \varepsilon_{q}^{d}=1=\varepsilon_{q}^{3 b+d}=1$. Portanto $3 b+d \equiv 0 \bmod 7$

de (2) $\varepsilon_{1}^{2 b} \cdot \varepsilon_{1}^{3 d}=1 \Rightarrow \varepsilon_{1}^{2 b+3 d}=1$. Portanto $2 b+3 d \equiv 0 \bmod 7$ (5)

de (4) $d \equiv-3 b \bmod 7$ ou $d \equiv 4 b \bmod 7$ que em (5) nos fornece:

$2 b+12 a \equiv 0 \bmod 7$, que sempre se verfica.

Assim temos 7 (sete) soluções possíveis para $\alpha$ e $\delta$.

\begin{tabular}{|llllllll|}
\hline $\mathrm{b}$ & 0 & 1 & 2 & 3 & 4 & 5 & 6 \\
\hline $\mathrm{d}$ & 0 & 4 & 1 & 5 & 2 & 6 & 3 \\
\hline
\end{tabular}

Portanto a substituição é da forma : $\left\{\begin{array}{c}x=\varepsilon_{1}{ }^{b} X \\ y=\varepsilon \\ { }_{1}{ }^{1}\end{array}\right.$ que na equação ,

fica: $X^{3} Y+a X^{2} Y^{3}+Y^{7}+c_{D} \varepsilon_{i}^{b}\left(\varepsilon_{1}{ }^{d}\right)^{6} X Y^{6}$

porem $b+6 d \equiv b+24 b \equiv 25 b \equiv 4 b \equiv d \bmod 7$. Logo, ficamos com:

$x^{3} y+a x^{2} y^{3}+y^{7}+c_{0} \varepsilon_{1}^{d} x y^{6}$ com $d=0,1,2, \ldots, 6$

Consideremos agora a substituição

$$
\left\{\begin{array}{l}
\mathrm{x}=\alpha X+B Y^{2} \\
\mathrm{y}=\delta Y
\end{array}\right.
$$

$\left(\alpha X+\beta Y^{2}\right)^{3}(\delta Y)+a\left(\alpha X+\beta Y^{2}\right)^{2}(\delta Y)^{3}+(\delta Y)^{7}=$ 
De $(8) \delta^{2}=-\alpha$ em (5) $\beta=-\frac{2}{3} a^{\delta^{2}}=\frac{2}{3} a \alpha=-\frac{2}{3 \sqrt{2}} \varepsilon i^{\alpha}$

\section{Rntäa a audancra é da forma}
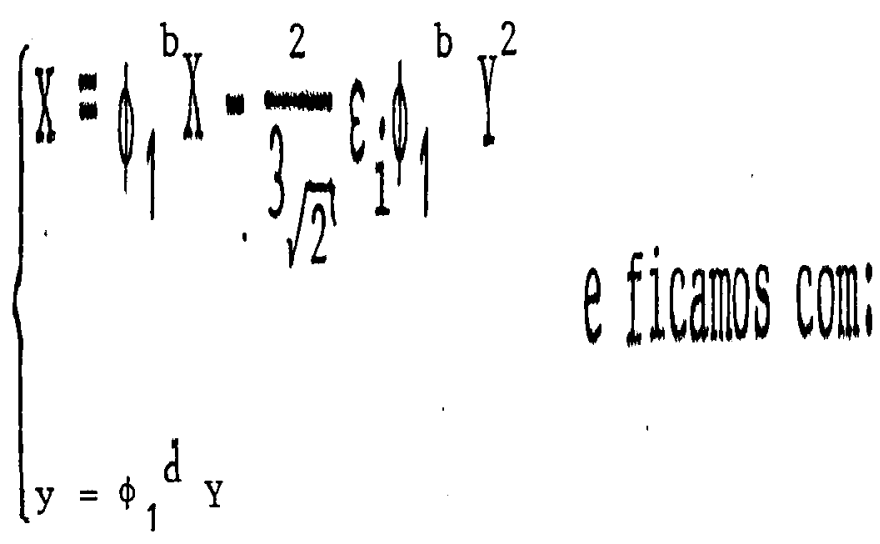

$X^{3} Y-\frac{3}{3 \sqrt{2}} \varepsilon_{i} X^{2} Y^{3}+Y^{7}+c_{o}\left(\phi_{1}^{b} X-\frac{2}{3 \sqrt{2}} \varepsilon_{i} \phi_{1}^{b} Y^{2}\right)\left(\phi_{1}{ }^{d} Y\right)^{6}$.

$A=c_{0} \phi_{1}{ }^{b} \phi_{1}{ }^{6 d} X Y^{6}-\frac{2}{3 \sqrt{2}} c_{o} \phi_{1}{ }^{b} \phi_{1}{ }^{6 d} \varepsilon_{i} Y^{8}$, mas observemos que:

$a+6 d \equiv b+66 b \equiv 67 b \equiv 11 b \equiv d \bmod 14$. Portanto:

$A=c_{0} \delta X Y^{6}-\frac{2}{3 \sqrt{2}} c_{0} \delta \varepsilon_{i} Y^{8}$

Porem: $f_{0}=X^{3} Y-\frac{3}{3 \sqrt{2}} \varepsilon_{i} X^{2} Y^{3}+Y^{7}\left\{\begin{array}{l}\partial_{1} f_{0}=3 X^{2} Y-\frac{6}{3 \sqrt{2}} \varepsilon_{i} X Y^{3} \\ \partial_{2} f_{0}=X^{3}-\frac{9}{3 \sqrt{2}} \varepsilon_{i} X^{2} Y^{2}+7 Y^{6}\end{array}\right.$

E assim: $Y^{8}=\frac{1}{7} Y^{2} \partial_{2} f_{0}+\frac{3}{7^{3} \sqrt{2}} \varepsilon_{i} Y^{3} \partial_{1} f_{0}-X Y \partial_{1} f_{0}-$

$$
-\frac{2}{21^{3} \sqrt{2}} \varepsilon_{i} \mathrm{Y}^{3} \partial_{1} \mathrm{f}_{\mathrm{o}}-\frac{14}{7 \sqrt[3]{4}} \varepsilon_{i}^{2} \mathrm{XY}^{6}
$$

Portanto $A=c_{0} \delta X Y^{6}-2 c_{0} \delta X Y^{6}=-c_{0} \phi_{1}{ }^{d} X Y^{6}$.

Concluímos que no caso $i=1$ o difeomorfismo permitido, leva $f^{\prime}$ em 
$\left\{\begin{array}{l}x=\alpha X \\ y=\delta Y\end{array}\right.$ em $f_{0}$, obtemos então:

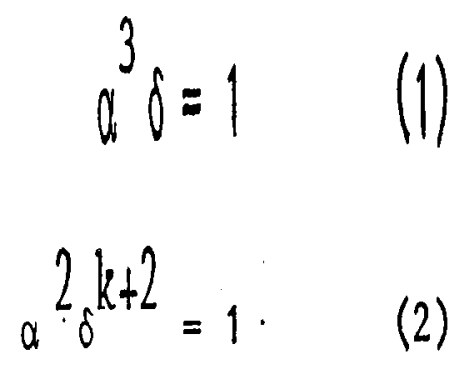

$\delta^{3 k+4}=1$

De (1) em (2) $\alpha^{2} \cdot\left(1 / \alpha^{3}\right)^{k+2}=1 \Rightarrow \alpha^{2}=\alpha^{3 k+6} \Rightarrow \alpha^{2}\left(1-\alpha^{3 k+4}\right)=0$

Portanto:

$$
\alpha^{3 k+4}=1
$$

Seja $\varepsilon_{1}$ a primeira das raizes $(3 k+4)$-ésimas da unidade, façamos:

$$
\alpha=\varepsilon_{q}^{b}: \text { e } \delta=\varepsilon_{q}^{d}
$$

em (1) $\varepsilon_{1}^{3 b} \cdot \varepsilon_{1}{ }^{d}=1 \Rightarrow 3 b+d \equiv 0 \bmod (3 k+4)$

em (2) $\varepsilon_{1}{ }^{2 b} \cdot \varepsilon_{1}{ }^{(k+2) d}=1 \Rightarrow 2 b+(k+2) d \equiv 0 \bmod (3 k+4)$

de (5) $d \equiv[(3 k+4)-3] b \bmod (3 k+4) \Rightarrow d \equiv(3 k+1) b \bmod (3 k+4)$ em $(6)$ $2 b+(k+2) d \equiv 2 b+(k+2)(3 k+1) b \equiv\left(2+(3 k+1)(k+2) b \equiv\left(3 k^{2}+7 k+4\right) b \equiv\right.$

$\equiv(3 k+4)(k+1) b \equiv 0 \bmod (3 k+4)$

$$
\text { Logo, temos }(3 k+4) \text { soluçōes possíveis para } \alpha \text { e } \delta \text {. }
$$


$b+(2 k+5) d \equiv b+(3 k+1)(2 k+5) b \equiv\left(1+6 k^{2}+17 k+5\right) b \equiv\left(6 k^{2}+17 k+6\right) b \equiv(3 k+2) b \equiv$

(*)

$\equiv 2 d \bmod (3 k+4)$

(*) se verifica, De fato:

$$
\begin{aligned}
& \text { Como } d \equiv(3 k+1) b \bmod (3 k+4) \Rightarrow \frac{d-(3 k+1)}{3 k+4}=w \subset z \Rightarrow \\
& \Rightarrow \frac{2 d-2(3 k+1)}{3 k+4}=2 w \Rightarrow 2 d \equiv 6 k+2 \equiv(3 k-2) \bmod (3 k+4) \\
& \vdots \\
& b+(3 k+3) d \equiv b+(3 k+1)(3 k+3) b \equiv\left(1+9 k^{2}+12 k+3\right) b \equiv\left(9 k^{2}+12 k+4\right) b \equiv 4 b \stackrel{(* *)}{ } \equiv \\
& \equiv k d \bmod (3 k+4) \\
& \text { (**) se verifica. De fato: }
\end{aligned}
$$

Comod $d \equiv(3 k+1) b \bmod (3 k+4) \Rightarrow k d \equiv\left(3 k^{2}+k\right) b \equiv 4 b \bmod (3 k+4)$ Assim ficamos com:

$$
x^{3} y+a x^{2} y^{k+2}+y^{3 k+4}+c_{0} \varepsilon_{1}^{d} x y^{2 k+4}+c_{1} \varepsilon_{1}^{2 d} x y^{2 k+5}+\ldots+c_{k-1} \varepsilon_{1}^{k d} x y^{3 k+3} .
$$


$\left\{\begin{array}{l}x=\phi_{1}^{b} X-\frac{2}{3} \varepsilon_{1} \phi_{1}^{b} Y^{k+1} \\ y=\phi_{1}^{d} Y\end{array}\right.$

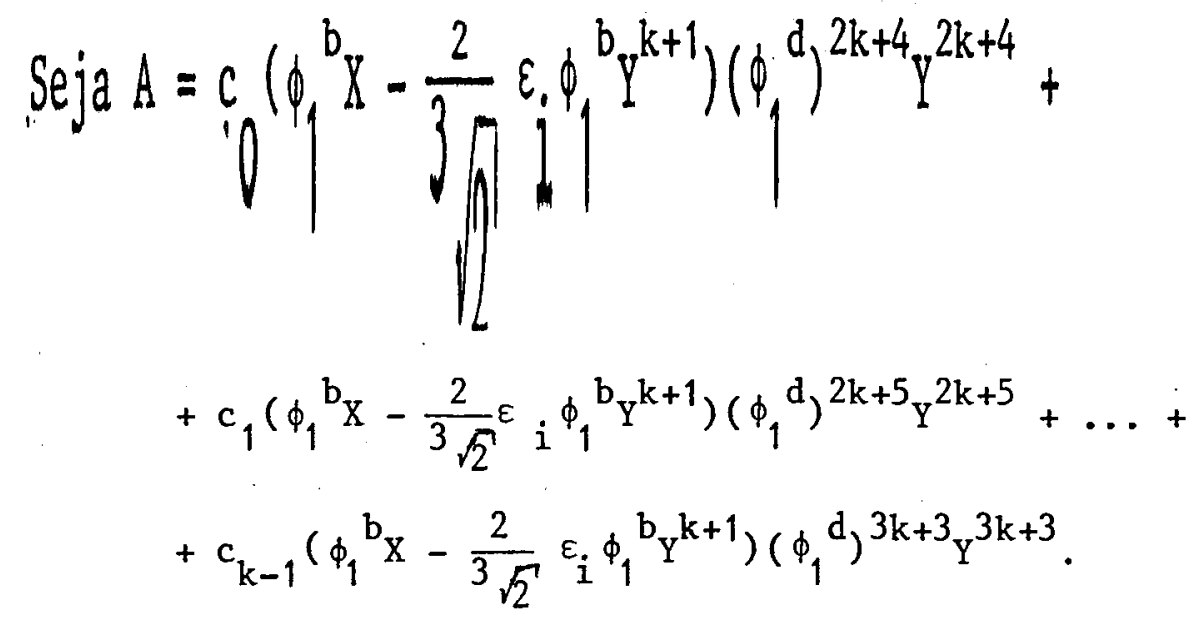

Mas : $\quad a+(2 k+4) d \equiv d \bmod (6 k+8)$

$a+(2 k+5) d \equiv 2 d \bmod (6 k+8)$

$a+(3 k+3) d \equiv k d \bmod (6 k+8)$

(demonstrado anteriormente um caso anälogo)

Portanto $A=c_{0} \delta\left(X-\frac{2}{3 \sqrt{2}} \varepsilon i^{k+1}\right) Y^{2 k+4}+c_{1} \delta^{2}\left(X-\frac{2}{3 \sqrt{2}} \varepsilon i^{Y^{k+1}}\right) Y^{2 k+5}+$

$+\ldots+c_{k-1} \delta^{k}\left(X-\frac{2}{3 \sqrt{2}} \varepsilon_{i} Y^{k+1}\right) Y^{3 k+3}$

Porem $f_{0}=X^{3} Y-\frac{3}{\sqrt[3]{2}} \varepsilon_{i} X^{2} Y^{k+2}+Y^{3 k+4} \cdot$ Logo

$\partial f_{0}=3 X^{2} Y-\frac{6}{3 \sqrt{2}} \varepsilon_{i} X Y^{k+2}$

$\partial_{2} f_{0}=X^{3}-\frac{3(k+2)}{\sqrt[3]{2}} \varepsilon_{i} X^{2} Y^{k+1}+(3 k+4) Y^{3 k+3}$

E assim: 


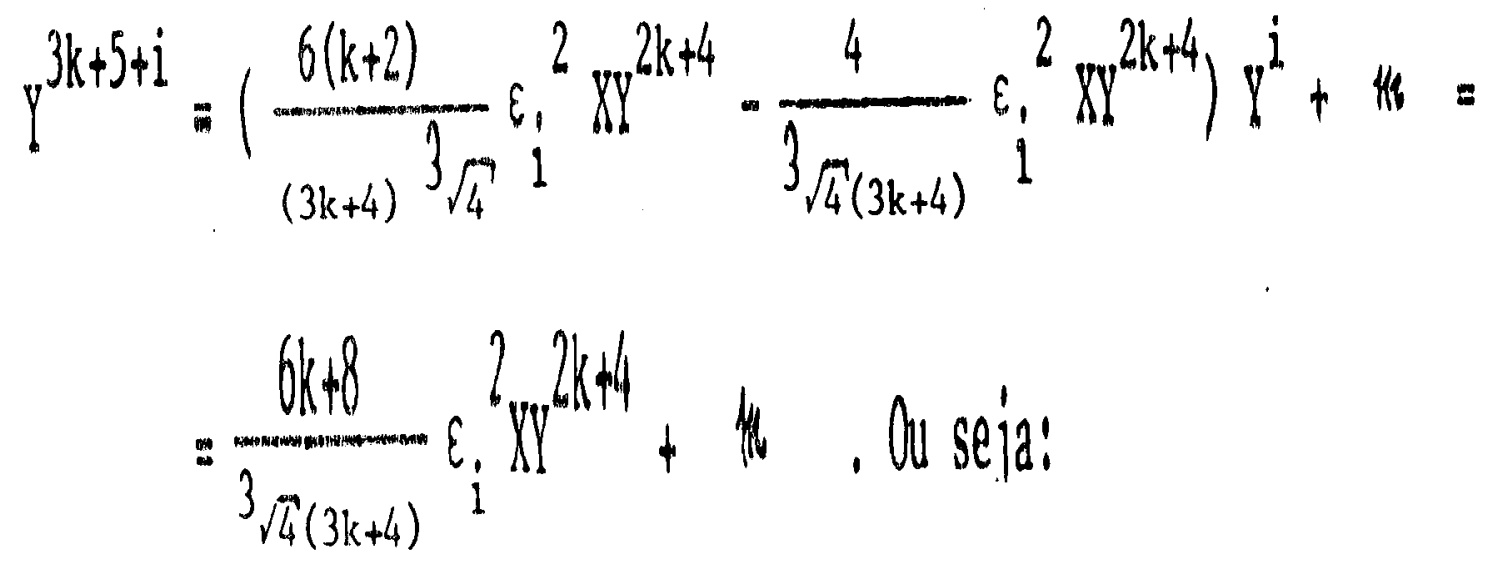

$A=\sum_{i=0}^{k-1} c_{1} d_{i}^{d i} x y^{2 k+4+i}$

Logo difeomorfismos que fixam $f_{0}$ nos fornecem $6 k+8$ elementos $f^{\prime}$ nu ma mesma örbita.

Seria interessante ter estudado a topologia da parte quasehomogê nea $f_{o}$ deste último exemplo e tambèm o comportamento de $f^{\prime}$ quando aplicamos difeomorfismos que fixam $f_{0}$ a menos de germes de uma mesma örbita, mas isto ampliaria bastante o trabalho, pois é um caso com um pouco mais de detalhes, porisso deixamos para uma proxima oportunidade. 
[1] - ARNOL'D, V.I., Critical points of smooth functions and their normal forms. Russian Math. Surveys, 30:5 (1975), 1-75.

= Uspekhi Mat. Nauk, 30:5 (1975), 3-65.

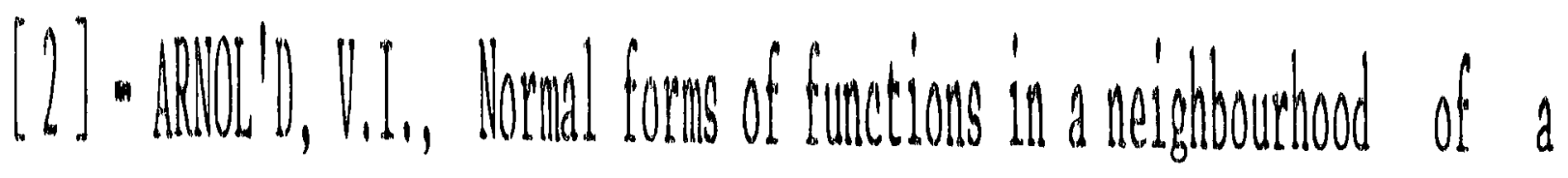
degenerate critical point. Uspekhi Mat. Nauk, 29:2 (1974), 11-49. = Russian Math. Surveys, 29:2 (1974), 10-50.

[3] - ARNOL'D, V.I., Normal forms for functions near degenerate critical points, the Weyl groups of $A_{k}, D_{k}, E_{k}$ and Lagrangian singularities. Funktsional'nyi Analiz i Ego Prilozheniya, Vol. 6, n. ${ }^{\circ} 4$ (1972), 3-25; MR $50 \# 8595$.

= Functional Anal. Appl. 6 (1973), 254-272.

[4] - HUMPHREYS; ME., Introductions to Lie Algebras and Representations Theory. Second Printing, Revised 1972, Springer-Verlag, New York Inc. (GTM-09).

[5] - MATHER, I.N., Stability of $C^{\infty}$-Mappings: finitely determined map-germs. Publ. Math. I.H.E.S., Vol. 35, 1968.

[6] - BRÖCKER, Th., Differentiable Germs and Catastrophes. Cambridge University Press, 1975. (LMS-17).

[7] - GIBSON, C.G., Singular Points of Smooth Mappings. Pitman Publishing Limited, 1979.

[ 8 ] - GOLUBitskY, M. \& GUILlemin, V., Stable Mappings and Their Singularities. Springer-Verlag, 1973. (GTM-14). 